\title{
NUTRITION, HEALTH AND MICROBIAL ECOLOGY OF TRADITIONAL FERMENTED FOODS IN ZAMBIA
}

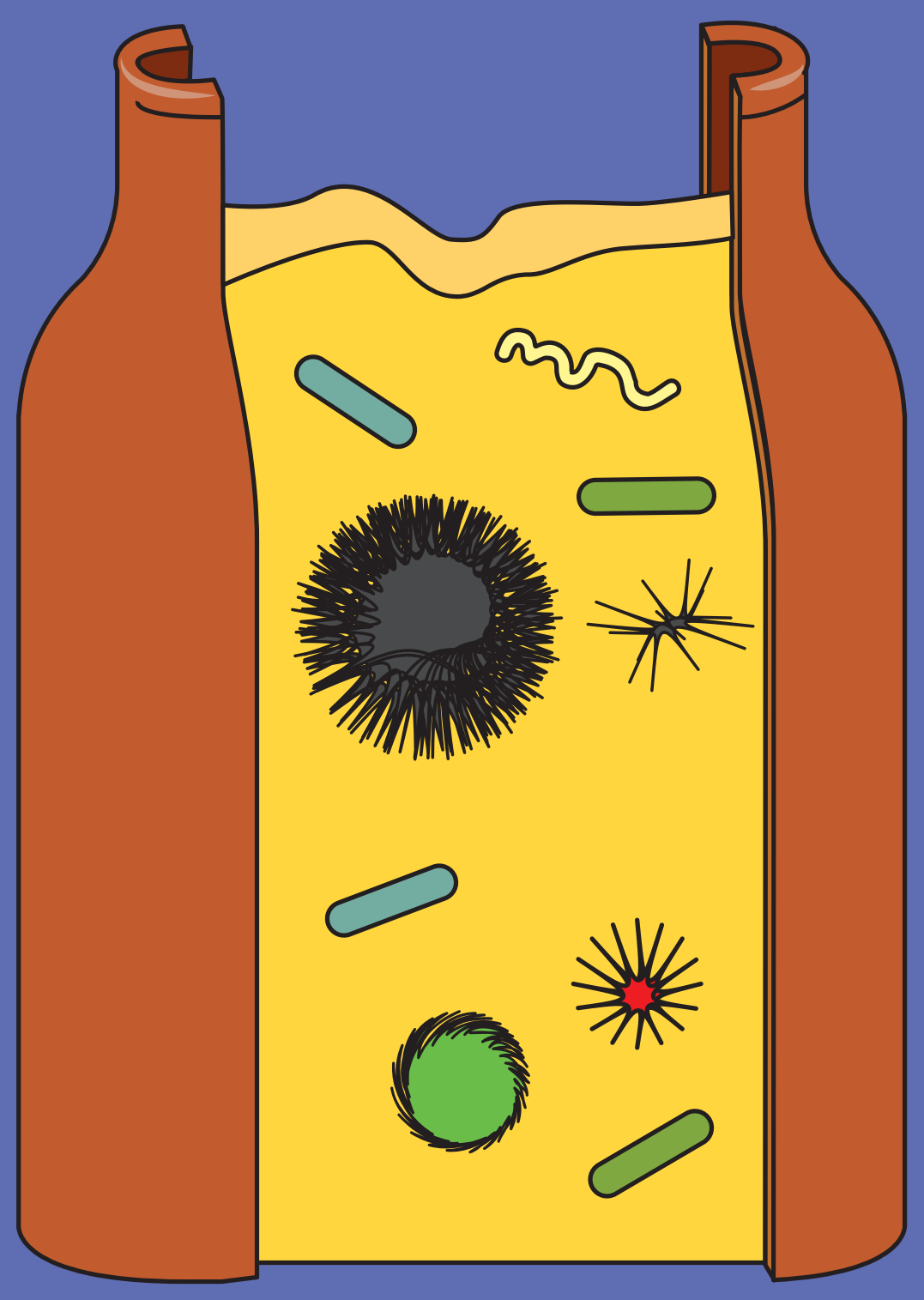

Justin Chileshe 
Nutrition, health and microbial ecology of traditional fermented foods in Zambia 


\section{Thesis committee}

\section{Promotor}

Prof. Dr B.J. Zwaan

Professor of Genetics

Wageningen University \& Research

\section{Co-promotors}

Dr S.E. Schoustra

Assistant professor, Laboratory of Genetics

Wageningen University \& Research

Visiting professor at the Department of Food Science and Nutrition

University of Zambia

Dr E.F. Talsma

Assistant professor, Human Nutrition and Health

Wageningen University \& Research

\section{Other members}

Prof. Dr Vincenzo Fogliano, Wageningen University \& Research

Dr Christele Humblot, IRD Montpellier, France

Prof. Dr Remco Kort, VU Amsterdam

Dr Clara Belzer, Wageningen University \& Research

This research was conducted under the auspices of the Graduate School for Product Ecology and Resource Conservation. 


\title{
Nutrition, health and microbial ecology of traditional fermented foods in Zambia
}

\author{
Justin Chileshe
}

Thesis

Submitted in fulfilment of the requirements for the degree of doctor at Wageningen University by the authority of the Rector Magnificus,

Prof. Dr A.P.J. Mol, in the presence of the Thesis Committee appointed by the Academic Board to be defended in public on Monday 21 October 2019 at 1:30 PM in the Aula. 
Justin Chileshe

Nutrition, health and microbial ecology of traditional fermented foods in Zambia 141 Pages

PhD Thesis, Wageningen University, Wageningen, The Netherlands (2019)

With references, with summary in English

ISBN 978-94-6395-110-4

DOI https://doi.org/10.18174/499123 


\section{Table of Contents}

\section{Chapter 1}

General Introduction

\section{Chapter 2}

Potential contribution of cereal and milk based fermented foods to dietary

nutrient intake of 1-5 years old children in Central province in Zambia.

\section{Chapter 3}

Nutritional composition and microbial communities of two non-alcoholic

traditional fermented beverages from Zambia: A study of Mabisi and Munkoyo

\section{Chapter 4}

Do traditional fermented foods affect the gut microbiota metabolism?

Assessing the potential using an in vitro system

\section{Chapter 5}

Effects of traditional fermented foods consumption on gut microbial composition in 6-24 months old children in rural Zambia

\section{Chapter 6}

General Discussion

Summary

Acknowledgements

List of Publications

About the Author

Overview of completed training activities 
Chapter 1 
General Introduction

Justin Chileshe 


\section{Introduction}

\section{Scope}

The widespread societal, development and health problem of undernutrition in many developing countries motivated my research. Despite past interventions and economic developments, many developing countries still faces high undernutrition levels especially stunting (linear growth failure), deficiencies in zinc, vitamins A and B12, and folate, mainly in children under the age of five years [1, 2]. Some of the current and proposed interventions include dietary diversification, sugar fortification with vitamin A, high dose vitamin A capsule distribution to under five children twice every year, maize meal fortification, and others. Dietary diversification and improvement in the food preparation methods such as fermentation are areas with potential to improve availability of nutrients especially for vulnerable populations. For this thesis, the focus is on Zambia, which is one of the most undernourished countries in the world, with $48 \%$ of the population considered undernourished [3]. As Zambia is dependent on mainly maize as a staple food with lower consumption of animal food sources, diversification of diets presents an opportunity to combat undernutrition in this population. Within this, the traditional use of fermented foods presents an opportunity for improvement in nutrient intake in the population especially of children below the age of five years [4, 5]. My thesis explores this potential.

\section{Undernutrition}

Undernutrition is a global problem that results from inadequate food intake and the consequences of repeated infections leading to especially stunting and micronutrient deficiencies. Stunting in childhood is the most prevalent form of undernutrition globally affecting an estimated 165 million children under five years old that experience life-long consequences of low height for weight and other health related effects [6]. Zambia is among the five most affected countries worldwide. Micronutrient deficiencies are also prevalent affecting around two billion people globally with the most common deficiencies in vitamin A, folate, iron, iodine, and zinc [7]. Undernutrition is responsible for $45 \%$ of deaths of children younger than five years, amounting to more than 3 million deaths each year worldwide [8]. Childhood stunting is also an outcome of maternal under nutrition and inadequate infant and young child feeding (IYCF; [9]). The intrauterine period and first six months of life are critical for the development of stunting whereas the subsequent year is more critical mainly for the development of underweight and wasting (i.e. low weight for height; $[9,10]$ ).

According to the United Nations Children's Fund (UNICEF) conceptual framework (Figure 1; [11]), undernutrition results from a number of factors at defined as basis, underlying and immediate causes 
that affect both mother and child and that result in short and long term consequences. Some of the short-term consequences of undernutrition in children include greater susceptibility to infections, higher risk of mortality and disability, while long-term consequences include reduced growth, cognitive development impairment, reduced economic opportunity, effect on reproductive health, overweight, obesity, and non-communicable diseases $[7,12,13]$. The cross-cutting importance of nutrition has been reaffirmed in recent years by various global agencies, notably, the World Bank, the World Health Organization (WHO), the Food and Agriculture Organization (FAO), and the United Nations Children's Fund (UNICEF) [14, 15]. UNICEF and WHO emphasizes the importance of adequate nutrition in the 1000-day window (from conception to 24 months of age) when the foundation is laid for an individual's physical size as well as their physiological and intellectual capacities in later life [11].

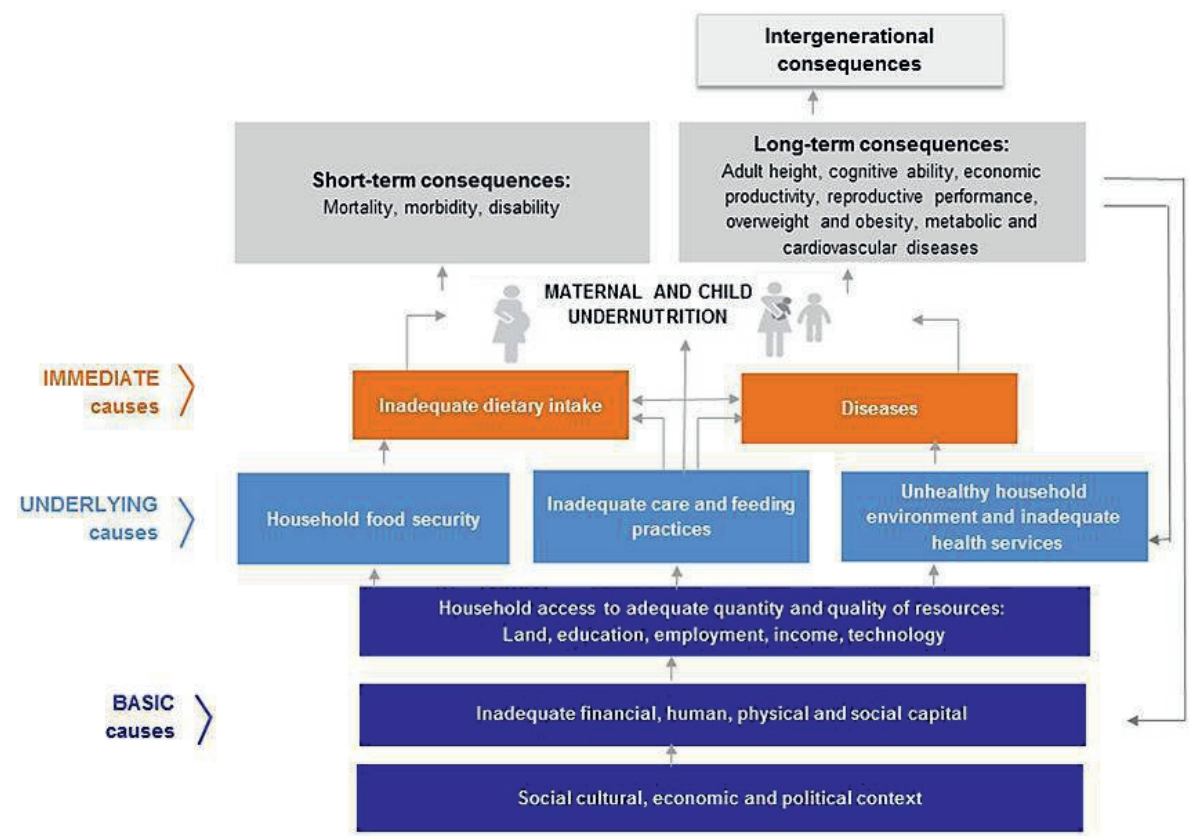

Figure 1: The UNICEF conceptual framework of causes and consequences of undernutrition. The black arrows show that the consequences of undernutrition can feed back to the underlying and basic causes of undernutrition, perpetuating the cycle of undernutrition, poverty and inequities [11].

In sub-Saharan Africa, chronic undernutrition has been a persistent problem especially in young children. This region has the one of the world's highest rates of stunting in children at $39 \%$ accounting for one third of all undernourished children globally. Africa is the only region that has recorded an 
increase in stunting levels from 50.3 million in the year 2000 to 58.8 million children under the age of five years in 2018 [16]. The figures for wasted children in the African region have also been high with $28 \%$ of the children under five years old wasted [16].

\section{Undernutrition in Zambia}

Zambia, located in Southern Africa (GPS coordinates: $13.1339^{\circ} \mathrm{S}, 27.8493^{\circ} \mathrm{E}$ ), has made great strides in economic development in recent years to the point that the World Bank reclassified the country in 2012 as lower middle-income. Despite this, Zambia is still among the group of countries suffering high levels of macro- and micronutrient deficiencies, particularly among young children and pregnant/lactating women $[17,18]$. Undernutrition is endemic in many parts of the country and poses a serious threat to the well-being of many, especially in the rural areas. It is responsible for $52 \%$ of all deaths occurring in children below the age of five years $[17,19]$. The most recent Zambia Demographic and Health Survey (ZDHS), conducted in 2013 revealed that $40 \%$ of children aged below five years were stunted recording a slight decrease from $45 \%$ in $2007,6 \%$ wasted, and $15 \%$ underweight which figures have remained the same since 2007 [20].

Zambia has adopted parts of a package defined by the World Health Organisation (WHO) and other United Nations agencies of effective nutrition interventions to reduce the levels of stunting and undernutrition [21]. The package includes adequate maternal nutrition during pregnancy and lactation, early initiation of breastfeeding, exclusive breastfeeding for the first six months, continued breastfeeding and adequate complementary feeding from six to 24 months, and increased micronutrient intakes during the critical 1000 days [21]. Zambia has had some of the interventions in place to reduce undernutrition for some time now. Impact has been recorded in some areas such as decreased underweight from $23 \%$ in 2002 to $15 \%$ in 2014, decreased iodine deficiency from $72 \%$ in 1992 to $4 \%$ in 2003, and increased rates of exclusive breast feeding from 10\% in 1992 to $73 \%$ in 2014 [20]. Vitamin A deficiency (VAD) and anaemia have been reduced though they remain high in children under five at 49\% VAD in the 2009 survey of eastern and central provinces, and 55\% anaemia in 2012 $[2,22]$. Some of the interventions targeting micronutrients deficiencies of, for example, vitamin $A$, iron, and iodine have been given more attention.

Recent economic advancement and specific intervention programs have thus far not led to lower levels of undernutrition possibly due to limited access to health services, limited access to food affecting diversity in the diet and limited improvement in water and sanitation services especially for the rural population among others [23]. Much as the importance of such micronutrients is appreciated, a more holistic approach to promoting and putting in place interventions that target even other nutrients 
would go a long way. There has been more dependence for example on a few staple crops such that even consumption of food has been limited to the more common cereal crops such as maize that do contain high quality carbohydrates yet are not rich in micronutrients [4].

\section{Use of local foods as a bottom-up approach to combat undernutrition}

Local foods other than the commonly used cereal crops are underutilized as a means to combat undernutrition. The increased use of maize in current diets has decreased the use of other local foods that could diversify diets and are in principle acceptable to consumers due to their status as traditional foods. Local foods have however not been evaluated for their nutritional composition and are in many cases not included in the design of programs aimed to enrich current diets with nutrient rich foods. A special class of traditional local foods includes fermented foods, that are commonly valued for their preservation of raw materials and improvement of organoleptic properties of the raw materials through the addition of aroma and flavour. In the context of nutrition, fermented foods are of special interest since they do not only have the potential to enrich current diets with nutrients, but also to confer direct health benefits based on their probiotic effects [24-26]. Most fermented products are acidic (non-alcoholic) and are traditionally consumed by the entire population, including young children $[25,27]$. Utilisation of food preparation methods such as fermentation that make nutrients in such foods available is then potentially one of the best approaches that can help improve health of vulnerable populations such as children in their first 1000 days of life. Part of the research described in my thesis therefore assesses the potential of traditional non-alcoholic fermented products to contribute to the reduction in undernutrition in Zambia, mainly focusing on children under five years of age.

\section{Fermented products}

Microbes have played an important role in human food production through fermentation since ancient times, originally for food preservation purposes. The technique of fermentation has been used globally to improve nutritional, safety, and organoleptic properties of food using different raw materials. Also in Africa the technique has been used as an inexpensive method to effectively preserve and improve sensory and safety of food [25]. The production of fermented products is based on the microbial activity that transforms a raw material into a product with increased sensory and nutritional value, leading to several known general nutritional attributes, including the following: 1) Enhanced nutritional value through the breakdown of certain constituents and anti-nutritional factors, increased availability of micronutrients such as iron, zinc and calcium, and the synthesis of $B$ vitamins [28, 29]. 2) Enhanced digestibility due to the breakdown of indigestible oligosaccharides such as lactose and complex polysaccharides [30]. 3) Enhanced food safety by protection from proliferation of pathogenic 
microbes by the low $\mathrm{pH}$ and antimicrobial compounds, which also gives the products a prolonged shelflife [31, 32]. 4) Elimination of toxic substances such as mycotoxins [33, 34]. 5) The fermenting bacteria serve as probiotics, contributing to a healthy ecology of intestinal bacteria which promotes general health [35-37].

Zambia has a wide range of local fermented foods and beverages similar to western yoghurt, wine, and beer. The common traditional non-alcoholic fermented beverages in Zambia include Mabisi and Munkoyo produced using traditional methods and are consumed by all age groups. One way of production is through transfer of a small fraction of the previous product into fresh raw material, a process called back-slopping. This method transfers the microbial communities underlying the fermentation from batch to batch, and another way of production is based on the natural spontaneous fermentation that utilizes bacteria from the environment [38]. Mabisi is made by placing fresh raw milk in a fermentation vessel and fermenting at ambient temperatures for 48 hours resulting in a mildly sour tasting product, as shown in figure $2 \mathrm{~B}$. Processing most notable differs in the repeated additions (or not) of fresh milk, the level of shaking during the fermentation, and the levels of back-slopping (transfer of material from a previous batch to a new batch) [39]. Munkoyo is made by mixing maize flour with water and boiled for several hours as depicted in figure 2A. After cooling, Rhynchosia roots are added to provide enzymes to degrade complex sugars and to provide a microbial inoculum for fermentation [40, 41]. Fermentation takes place in a variety of vessels at ambient temperatures lasting around 48 hours. Processing variations include the time allowed for cooking the maize porridge, the types of roots added, the fermentation vessel used, and the level of back-slopping [40]. For both Mabisi and Munkoyo products, the microbial communities responsible for fermentation are dominated by four to ten species of lactic acid and acetic acid bacteria but the exact composition varies between samples taken and differences in processing give rise to further variations in microbial composition $[27,39,40]$. 

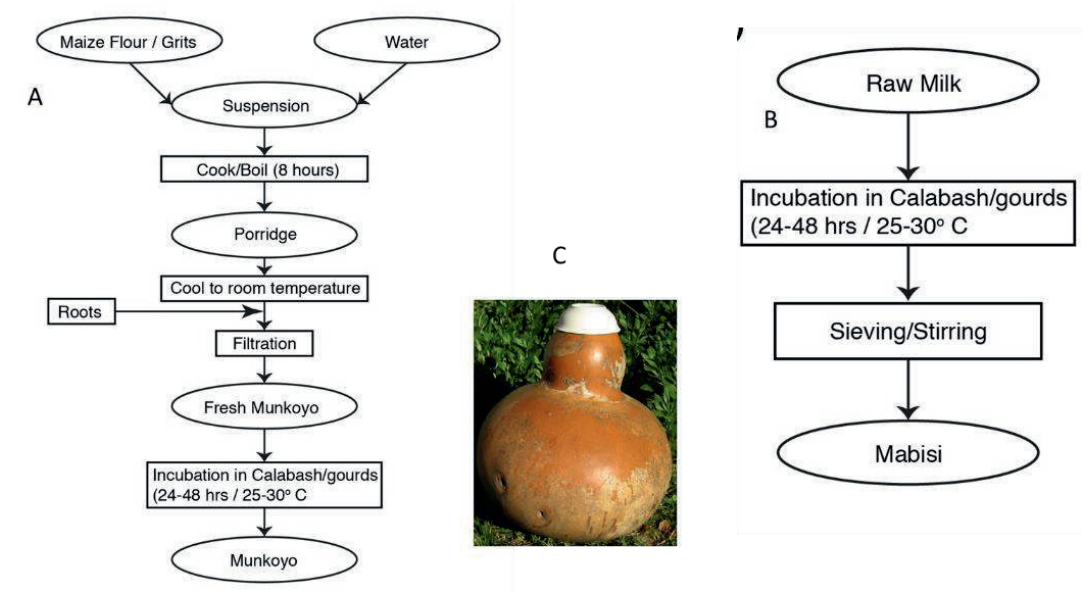

Figure 2: Shows the production procedure of Munkoyo (A), production procedure of Mabisi (B) and a calabash (C) used as one of the fermentation vessels. Adapted from Schoustra et al 2013 [27].

Producers are almost exclusively women, who make the products at home and sell at local markets generating a livelihood for themselves and their families. Previous work has shown that while the organoleptic properties of products made by various producers are similar, the microbial composition is highly variable from producer to producer [42]. Previous work on Mabisi and Munkoyo focussed on microbiology and certain biochemical properties [43,44]. However, there is still a striking lack of information regarding nutritional value of the Zambian traditional fermented foods which could be an important reason why the potential of these products to promote nutrition is currently unheeded. Indeed, the link between microbial composition and how these fermenting microbes may increase nutritional value of the raw materials adding to their nutritional potential has not been established.

Apart from direct nutritional benefits by adding nutrients to diets, local fermented foods may also have more indirect health benefits. The microbial communities in fermented products form an ecosystem and interaction with the gut microbiota (another ecosystem) may bring about shifts to healthier gut flora impacting on the health of hosts, as consumption of the fermented products has previously been shown [45]. This potential to invoke shifts in gut microbiota composition towards a healthier composition through the consumption of Mabisi or Munkoyo has to date not been explored nor studied.

\section{The Human Gut Microbiota}

The human gut is host to millions of bacteria and it is known that its composition is specific for every individual. However, most can be categorized as belonging to one of three groups, based on the predominance of either of the genera Bacteroides, Prevotella, or Ruminococcus [46]. Gut microbiota 
composition of humans is affected by changes in lifestyle and diet [47]. The intestinal microbiota has been recognised as a vital asset for health and neuro-development and is established in the first three years of life so that its modification during this period has the potential to affect host health and development [48]. It has been shown that shifts in microbiota composition towards more favourable taxa and combinations of taxa leads to better health, for instance by a better functioning immune system and protection against invasion of pathogenic bacteria via the intestine [49]. In this way, a healthy gut microbiota with good nutrition helps to prevent disease. In recent years it has also been shown that human microbiota perform several functions in the host including nutrition and metabolism, and structure and function of the gastro intestinal tract [50-52]. For example, the gut microbes synthesize micronutrients such as B vitamins and vitamin $\mathrm{K}$ which the humans cannot synthesise thus providing valuable benefits for both microbial and host metabolisms [53]. The human gut microbiota is involved in producing some of the dietary metabolites by fermenting complex starch and soluble dietary fibre in the colon [54]. An example of the fermentation end-products are the Short Chain Fatty Acids (SCFAs) such as butyrate, propionate, and acetate which contribute to the energy required for metabolic processes of the host [55].

Fermented foods contain classes of bacteria that are similar to those that are considered healthy within the human gut microbiota. For instance, fermented foods contain lactic acid bacteria of the genera, including Lactobacillus, Lactococcus, and Bifidobacterium [56, 57]. Based on this, these products could have probiotic effects by causing a shift in gut microbiota composition to a healthier balance. For several lactic acid bacteria, such as Lactobacillus rhamnosus GG and Lactobacillus casei, these probiotic properties have been formally demonstrated $[58,59]$. Since traditional fermented foods contain a mixed community of a variety of species, they are likely to include strains with probiotic effects that thus can have an impact to better health of their consumers.

\section{Methods of assessing effects of fermented foods on gut microbiota}

Different methods for assessing effects of fermented foods on gut microbiota exist ranging from flask based in vitro models, artificial gut systems, and in vivo using humans and animals. Human studies are the more insightful and are considered the gold standard, physiologically more relevant for studies of interactions between intestinal microbiota and probiotics for example, but are affected by technical, logistical, and ethical limitations [60]. This therefore has led to the use of animal and in vitro models that have been very useful for establishing cause and effect and to provide proof of principle demonstrating potential beneficial effects of interventions. At the same time, differences in physiology between humans and animal models disadvantages the latter compared to in vitro models because these also eliminate host factor interference [61]. The in vitro models that are commonly used are the less sophisticated batch fermentation since the growth of a pure or mixed bacterial suspension under 
a wide range of selected media and conditions is possible [60]. Use of this batch fermentation to depict the human gut requires controlling the conditions such as anaerobic atmosphere, physiological temperature, and $\mathrm{pH}$ [60]. A number of studies on dietary components have been conducted using this system such as investigation of resistant starch and carbohydrates as potential prebiotics [62], and investigation of metabolic profiles of SCFAs produced through metabolism of dietary compounds [63].

\section{Rationale and Outline of the thesis}

The research described in this thesis is centred around the potential of traditional non-alcoholic fermented foods, Mabisi and Munkoyo in the alleviation of undernutrition and in the promotion of better nutritional status. The prevalence and popularity of traditional dairy and cereal-based fermented food products present timely, favourable, yet currently underexploited opportunities to directly address undernutrition. While these products are widely produced and consumed by local people in Zambia, to date very little is known about their exact processing, food safety, and their nutritional and microbial composition. In addition, globalization has led to the availability of novel products from the international market (such as Coca Cola), that by some are perceived to be healthier and more fashionable than local traditional products, putting the consumption of traditional fermented products on a decline. These traditional fermented products have the potential to impact on the human gut microbiota and general health through the ingestion of beneficial bacteria. Interest on the human gut microbiota has been growing regarding its composition and metabolic activity and how it is modulated by diet and other factors [64]. A number of studies has been conducted on fermented foods mainly produced using starter cultures with specific types of bacteria to determine impact on the gut microbiota mostly in adult populations [65-84]. There is scanty information on research focussing on traditional non-alcoholic fermented beverages to determine their potential impact on gut microbiota and nutritional status. Taken together, this has the consequence that the nutritional potential of these traditional products that fit in the local culture is currently neglected and underutilised. Therefore, as explained in Figure 2, this thesis was setup to generate data to address the questions on nutritional and microbial potential of Mabisi and Munkoyo to improve diets and promote healthier gut microbiota composition that can have an impact on reducing undernutrition. This was aimed to show that traditional fermented foods could help as part of the solutions in addressing the inadequate dietary intake as immediate causes of undernutrition (Figure 1). 


\section{Inadequate dietary intake}

Underlying causes

Diets do not meet requirements Suboptimal health parameters

Opportunity: Local fermented foods to improve diets and health parameters

Optimization of
current diets using
available foods (Ch2)

Nutritional and microbial compositon of local fermented foods (Ch3)

\section{Improved nutritional status}

Assessment of potential of local fermented foods to improve gut microbiota composition (Ch4)

Improved gut microbiota composition
Survey of gut microbiota compostion and metabolism relation to current consumption (Ch5)

\section{Better health of currently undernourished populations}

Figure 2: Conceptual framework describing the setup of the research in this thesis with the aim to improving nutritional status and general health. Potential of fermented beverages (Mabisi and Munkoyo) in Zambia were assessed by firstly modelling through combining of local foods with and without addition of the fermented foods for improvement of nutrient intake. The fermented foods were then assessed for their nutritional and microbial composition. Finally effect of consuming fermented beverages on gut microbiota and metabolites through a laboratory experiment was conducted and a survey to determine differences in SCFAs and gut microbial composition between fermented beverages consumers and nonconsumers was determined in children 6-24 months of age. [Ch2 is chapter 2, Ch3 is chapter 3, Ch4 is chapter 4 and Ch5 is chapter 5.]

This research has three central aims. (1) Elucidating the nutritional potential of the Mabisi and Munkoyo to complement the diet of vulnerable groups such as children under five years old (Chapter 2). Specifically, we address the potential of fermented foods through modelling to show whether these foods can potentially fill nutrient gaps. (2) Determining the nutritional aspects and microbial composition of the target traditional fermented beverages Mabisi and Munkoyo and whether microbes influence the level of nutrients in the two products (Chapter 3). (3) Understanding the influence of the favourable bacteria in the products in shaping human gut microbiota towards more favourable composition and its impact on health (Chapters 4 and 5). Here we use an in vitro experiment and a cross-sectional survey with children to specifically determine the differences in gut microbes, and nutritional status between consumers and non-consumers of Mabisi and Munkoyo.

In Chapter $\mathbf{2}$ I describe the potential contribution of Mabisi and Munkoyo to nutrient intake in children under the age of five years using Optifood, a linear programming software that was developed by WHO 
and partners. Secondary dietary intake data collected using 24-hour recall method was modeled to develop food based recommendations FBRs, for children aged 1-3 and 4-5 years, respectively in Mkushi district of Zambia. Three scenarios per age group were modeled to determine weekly food-based recommendations based on: (1) food based recommendations with the local available foods, (2) food based recommendations with inclusion of Mabisi, a fermented milk beverage, and (3) food based recommendations with inclusion of Munkoyo, a cereal fermented beverage. The scenarios were compared to assess whether food based recommendations with the addition of Mabisi or Munkoyo achieved better nutrient intake.

Chapter 3 describes the results obtained from laboratory analysis of Mabisi and Munkoyo samples collected during a cross sectional study in Mkushi to determine their nutritional composition and microbial communities. I compared community structures in the two types of traditional fermented foods (Mabisi and Munkoyo) from different producers sampled from the same environment using culture independent method based on 16SrRNA sequencing. It was hypothesized that Mabisi and Munkoyo each contain distinct microbial communities and that nutritional content depends on microbial composition. I therefore addressed the following questions: Does different raw materials shape structure of microbial communities by filtering microbes from the environment, and is variation in nutritional value explained by difference in microbial community structure?

Chapter 4 describes an in vitro experiment on the impact of Mabisi and Munkoyo on the gut microbiota focusing on the potential changes in metabolite profiles of gut microbiota taken from stool samples upon exposure to Mabisi and Munkoyo. I further correlated these shifts in metabolite profiles to changes in abundance of a key indicator bacterium for healthy gut microbiota composition.

Chapter 5 describes the survey that was undertaken in Namwala and Mkushi to determine the potential effect of consuming traditional fermented foods on gut microbial composition, nutritional status and health in children 6-24 months old in rural Zambia. We know that the human gut microbiota consists of a complex community of microorganisms which play a role in immunity, nutrition and metabolism, and structure and function of the gastro intestinal tract. We also know that diet is one of the influential ways to modulate the gut microbiota and that fermented foods have been found to impact on the microbiota of the host. I therefore collected stool samples of the children 6-24 months old in Mkushi and Namwala and analysed them for their composition of gut microbiota and concentrations of short chain fatty acids and linked this to data on consumption patterns of the different foods including fermented foods. The expectation was that consumers of fermented foods have a healthier gut microbiota composition and better nutritional status than individuals that do not consume fermented foods. 
Chapter 6 summarises the main findings and conclusions from the data-chapters, while giving a perspective on the potential of the consumption of traditional fermented foods to enrich diets and promote better nutritional status of its consumers. This chapter provides an outlook on future research that is needed to fulfil this potential within the context of combating undernutrition in Zambia and other developing countries.

\section{References}

1. Halimatou A, Kohler L, Taren D, Mofu M, Chileshe J, Kalungwana N: Zambia food consumption and micronutrient status survey report. Lusaka: National Food and Nutrition Commission DRAFT 2014.

2. Hotz C, Chileshe J, Siamusantu W, Palaniappan U, Kafwembe E: Vitamin A intake and infection are associated with plasma retinol among pre-school children in rural Zambia. Public health nutrition 2012, 15(9):1688-1696.

3. FAO, IFAD, WFP: The State of Food Insecurity in the World 2014. Strengthening the enabling environment for food security and nutrition. In. Rome: FAO; 2014.

4. Zhang Z, Goldsmith PD, Winter-Nelson A: The Importance of Animal Source Foods for Nutrient Sufficiency in the Developing World:The Zambia Scenario. Food and Nutrition Bulletin 2016, 37(3):303-316.

5. Hotz C, Gibson RS: Traditional food-processing and preparation practices to enhance the bioavailability of micronutrients in plant-based diets. The Journal of nutrition 2007, 137(4):10971100.

6. Prendergast AJ, Humphrey JH: The stunting syndrome in developing countries. Paediatr Int Child Health 2014, 34(4):250-265.

7. Bailey RL, West Jr KP, Black RE: The Epidemiology of Global Micronutrient Deficiencies. Annals of Nutrition and Metabolism 2015, 66(suppl 2)(Suppl. 2):22-33.

8. Black RE, Alderman H, Bhutta ZA, Gillespie S, Haddad L, Horton S, Lartey A, Mannar V, Ruel M, Victora CG et al: Maternal and child nutrition: Building momentum for impact. The Lancet 2013, 382(9890):372-375.

9. WHO: Childhood Stunting: Challenges and opportunities. Report of a Promoting Healthy Growth and Preventing Childhood Stunting colloquium. In.: World Health Organization; 2014.

10. Thakwalakwa C, Flax V, Maleta K, Ashorn P: Prevention and treatment of childhood malnutrition in rural Malawi: Lungwena nutrition studies. Malawi Medical Journal 2009, 21:116-119.

11. Fund UNCs: Improving Child Nutrition: The achievable imperative for global progress. 2013.

12. Reinhardt K, Fanzo J: Addressing chronic malnutrition through multi-sectoral, sustainable approaches: a review of the causes and consequences. Frontiers in nutrition 2014, 1:13.

13. Walker SP, Chang SM, Powell CA, Simonoff E, Grantham-McGregor SM: Early childhood stunting is associated with poor psychological functioning in late adolescence and effects are reduced by psychosocial stimulation. The Journal of nutrition 2007, 137(11):2464-2469.

14. UNICEF., Fund UNCS: The state of the world's children 2009: maternal and newborn health, vol. 9: Unicef; 2008.

15. Organization WH: Nutrition for health and development: a global agenda for combating malnutrition. In.: Geneva: World Health Organization; 2000.

16. UNICEF: Levels and trends in child malnutrition UNICEF-WHO-World Bank Group joint child malnutrition estimates: Key findings of the. In.: New York, NY: UNICEF, WHO \& World Bank Group; 2017.

17. Unicef: WHO \& The World Bank. 2012. Levels and trends in child malnutrition: Joint child malnutrition estimates 2016.

18. Harris J, Drimie S: Toward an integrated approach for addressing malnutrition in Zambia: a literature review and institutional analysis. 2012.

19. DFID U: The Politics of Poverty: Elites Citizens and States. In.; 2011.

20. Demographic Z: Health survey (ZDHS) 2013-2014. House hold population by Age and sex Lusaka: Zambia 2015. 
21. WHO: Essential nutrition actions: improving maternal, newborn, infant and young child health and nutrition. 2013.

22. NMCC: Zambia Malaria Indicator Survey 2012 Report. 2013.

23. Siamusantu W, Bader, E, Palma, G, Razès, M, Dop, MC.: Nutrition Country Profile for the Republic of Zambia, Rome, Italy: Country Profiles-Food and Agriculture Organization of the United Nations. 2009.

24. Conlon $\mathrm{M}$, Bird $\mathrm{A}$ : The impact of diet and lifestyle on gut microbiota and human health. Nutrients 2015, 7(1):17-44.

25. Anukam KC, Reid G: African traditional fermented foods and probiotics. Journal of medicinal food 2009, 12(6):1177-1184.

26. Egounlety M, Aworh O: Effect of soaking, dehulling, cooking and fermentation with Rhizopus oligosporus on the oligosaccharides, trypsin inhibitor, phytic acid and tannins of soybean (Glycine max Merr.), cowpea (Vigna unguiculata L. Walp) and groundbean (Macrotyloma geocarpa Harms). Journal of food engineering 2003, 56(2-3):249-254.

27. Schoustra SE, Kasase C, Toarta C, Kassen R, Poulain AJ: Microbial Community Structure of Three Traditional Zambian Fermented Products: Mabisi, Chibwantu and Munkoyo. PLOS ONE 2013, 8(5):e63948.

28. Egounlety M, Aworh OC, Akingbala JO, Houben JH, Nago MC: Nutritional and sensory evaluation of tempe-fortified maize-based weaning foods. International journal of food sciences and nutrition 2002, 53(1):15-27.

29. Hotz C, Gibson RS: Traditional food-processing and preparation practices to enhance the bioavailability of micronutrients in plant-based diets. Journal of Nutrition 2007, 137:1097-1100.

30. Nout MJR: Rich nutrition from the poorest - Cereal fermentations in Africa and Asia. Food Microbiology 2009, 26(7):685-692.

31. Nout MJR, Darkar PK, Beuchat LR: Indigenous fermented foods. In: Food Microbiology: Fundamentals and Frontiers Third Edition. Edited by Doyle MP, Beuchat LR. Washingon DC: ASM Press; 2007: $817-$ 835 .

32. Battcock M, Azam-Ali S: Fermented fruits and vegetables. A global perspective. Rome: FAO; 1998.

33. Mokoena MP, Chelule PK, Gqaleni N: Reduction of Fumonisin B1 and Zearalenone by Lactic Acid Bacteria in Fermented Maize Meal. Journal of Food Protection 2005, 68(10):2095-2099.

34. Shephard GS: Impact of mycotoxins on human health in developing countries. Food Additives \& Contaminants: Part A 2008, 25(2):146-151.

35. Zimmermann MB, Chassard C, Rohner F, N'Goran EK, Nindjin C, Dostal A, Utzinger J, Ghattas H, Lacroix C, Hurrell RF: The effects of iron fortification on the gut microbiota in African children: a randomized controlled trial in Côte d'Ivoire. The American Journal of Clinical Nutrition 2010, 92(6):1406-1415.

36. Kort R, Sybesma W: Probiotics for every body. Trends in Biotechnology 2012, 30(12):613-615.

37. Nout MJR, De Vos WM, Zwietering MH: Food Fermentation: Wageningen Academic Publishers; 2005.

38. Nout M: Accelerated natural lactic fermentation of cereal-based formulas at reduced water activity. International journal of food microbiology 1992, 16(4):313-322.

39. Moonga HB, Schoustra SE, Linnemann AR, Kuntashula E, Shindano J, Smid EJ: The art of mabisi production: A traditional fermented milk. PLOS ONE 2019, 14(3):e0213541.

40. Phiri S, Schoustra SE, Van den Heuvel J, Smid EJ, Shindano J, Linnemann AR: Fermented cereal-based Munkoyo beverage: processing practices, microbial diversity and aroma compounds. PLOS ONE 2019, submitted.

41. Foma RK, Destain J, Mobinzo PK, Kayisu K, Thonart P: Study of physicochemical parameters and spontaneous fermentation during traditional production of munkoyo, an indigenous beverage produced in Democratic Republic of Congo. Food Control 2012, 25(1):334-341.

42. Schoustra SE, Kasase C, Toarta C, Kassen R, Poulain AJ: Microbial Community Structure of Three Traditional Zambian Fermented Products: Mabisi, Chibwantu and Munkoyo. PloS one 2013, 8(5).

43. Zulu R, Dillon V, Owens J: Munkoyo beverage, a traditional Zambian fermented maize gruel using Rhynchosia root as amylase source. International journal of food microbiology 1997, 34(3):249-258.

44. Lovelace C: Zambian opaque maize beer and munkoyo. Handbook of Indigenous Fermented Foods (KH Steinkraus, ed) Marcel Dekker, New York 1977:435-439.

45. Sanlier N, Gokcen BB, Sezgin AC: Health benefits of fermented foods. Critical reviews in food science and nutrition 2019, 59(3):506-527.

46. Arumugam M, Raes J, Pelletier E, Le Paslier D, Yamada T, Mende DR, Fernandes GR, Tap J, Bruls T, Batto JM et al: Enterotypes of the human gut microbiome. Nature 2011, 473(7346):174-180. 
47. Derrien M, Veiga P: Rethinking Diet to Aid Human-Microbe Symbiosis. Trends in microbiology 2017, 25(2):100-112.

48. Rodríguez JM, Murphy K, Stanton C, Ross RP, Kober OI, Juge N, Avershina E, Rudi K, Narbad A, Jenmalm MC et al: The composition of the gut microbiota throughout life, with an emphasis on early life. 2015 2015, 26.

49. Conlon MA, Bird AR: The impact of diet and lifestyle on gut microbiota and human health. Nutrients 2014, 7(1):17-44.

50. Lazar V, Ditu L-M, Pircalabioru GG, Gheorghe I, Curutiu C, Holban AM, Picu A, Petcu L, Chifiriuc MC: Aspects of Gut Microbiota and Immune System Interactions in Infectious Diseases, Immunopathology, and Cancer. Frontiers in Immunology 2018, 9(1830).

51. Thursby E, Juge N: Introduction to the human gut microbiota. The Biochemical journal 2017, 474(11):1823-1836.

52. Ramakrishna BS: Role of the gut microbiota in human nutrition and metabolism. J Gastroenterol Hepatol 2013, 28 Suppl 4:9-17.

53. Rowland I, Gibson G, Heinken A, Scott K, Swann J, Thiele I, Tuohy K: Gut microbiota functions: metabolism of nutrients and other food components. European journal of nutrition 2018, 57(1):1-24.

54. Salminen S, Bouley C, Boutron MC, Cummings JH, Franck A, Gibson GR, Isolauri E, Moreau MC, Roberfroid M, Rowland I: Functional food science and gastrointestinal physiology and function. British Journal of Nutrition 1998, 80(S1):S147-S171.

55. Payne AN, Chassard C, Banz Y, Lacroix C: The composition and metabolic activity of child gut microbiota demonstrate differential adaptation to varied nutrient loads in an in vitro model of colonic fermentation. FEMS microbiology ecology 2012, 80(3):608-623.

56. Rezac S, Kok CR, Heermann M, Hutkins R: Fermented Foods as a Dietary Source of Live Organisms. Frontiers in microbiology 2018, 9:1785-1785.

57. Kho ZY, Lal SK: The Human Gut Microbiome - A Potential Controller of Wellness and Disease. Frontiers in Microbiology 2018, 9(1835).

58. Gosselink MP, Schouten WR, van Lieshout LM, Hop WC, Laman JD, Ruseler-van Embden JG: Delay of the first onset of pouchitis by oral intake of the probiotic strain Lactobacillus rhamnosus GG. Diseases of the colon \& rectum 2004, 47(6):876-884.

59. Vanderhoof JA, Whitney DB, Antonson DL, Hanner TL, Lupo JV, Young RJ: Lactobacillus GG in the prevention of antibiotic-associated diarrhea in children. The Journal of pediatrics 1999, 135(5):564568.

60. Takagi R, Sasaki K, Sasaki D, Fukuda I, Tanaka K, Yoshida K-i, Kondo A, Osawa R: A single-batch fermentation system to simulate human colonic microbiota for high-throughput evaluation of prebiotics. PLoS One 2016, 11(8):e0160533.

61. Fritz JV, Desai MS, Shah P, Schneider JG, Wilmes P: From meta-omics to causality: experimental models for human microbiome research. Microbiome 2013, 1(1):14.

62. Nicholson JK, Holmes E, Kinross J, Burcelin R, Gibson G, Jia W, Pettersson S: Host-Gut Microbiota Metabolic Interactions. Science 2012, 336(6086):1262-1267.

63. Bäckhed F, Fraser Claire M, Ringel Y, Sanders Mary E, Sartor RB, Sherman Philip M, Versalovic J, Young V, Finlay BB: Defining a Healthy Human Gut Microbiome: Current Concepts, Future Directions, and Clinical Applications. Cell Host Microbe 2012, 12(5):611-622.

64. Kho ZY, Lal SK: The Human Gut Microbiome - A Potential Controller of Wellness and Disease. Front Microbiol 2018, 9:1835-1835.

65. Macfarlane GT, Cummings JH: Probiotics, infection and immunity. Current opinion in infectious diseases 2002, 15(5):501-506.

66. Vitapole D: Fermented foods and healthy digestive functions: J. Libbey Eurotext; 2001.

67. Reid G, Bruce AW: Could probiotics be an option for treating and preventing urogenital infections? Medscape women's health 2001, 6(5):9-9.

68. Reid G, Beuerman D, Heinemann C, Bruce AW: Probiotic Lactobacillus dose required to restore and maintain a normal vaginal flora. FEMS Immunology \& Medical Microbiology 2001, 32(1):37-41.

69. Kyne L, Kelly C: Recurrent Clostridium difficilediarrhoea. Gut 2001, 49(1):152-153.

70. Guslandi M, Mezzi G, Sorghi M, Testoni PA: Saccharomyces boulardii in maintenance treatment of Crohn's disease. Digestive diseases and sciences 2000, 45(7):1462-1464.

71. Gorbach SL: Probiotics and gastrointestinal health. The American journal of gastroenterology 2000, 95(1):S2-S4. 
72. Gionchetti P, Rizzello F, Venturi A, Campieri M: Probiotics in infective diarrhoea and inflammatory bowel diseases. Journal of gastroenterology and hepatology 2000, 15(5):489-493.

73. Cunningham-Rundles S, Ahrné S, Bengmark S, Johann-Liang R, Marshall F, Metakis L, Califano C, Dunn A-M, Grassey C, Hinds G: Probiotics and immune response. The American journal of gastroenterology 2000, 95(1):S22-S25.

74. Caplan MS, Jilling T: Neonatal necrotizing enterocolitis: possible role of probiotic supplementation. Journal of pediatric gastroenterology and nutrition 2000, 30:S18-S22.

75. Bengmark S: Colonic food: pre-and probiotics. The American journal of gastroenterology 2000, 95(1):S5-S7.

76. Lee Y, Nomoto K, Salminen S, Gorbach S: Role of probiotics in health and diseases. Handbook of probiotics John Wiley \& Sons, Inc, New York, NY 1999:67-146.

77. Campieri M, Gionchetti P: Probiotics in inflammatory bowel disease: new insight to pathogenesis or a possible therapeutic alternative? In.: Elsevier; 1999.

78. Taranto MP, Medici M, Perdigon G, Ruiz Holgado AP, Valdez GF: Evidence for hypocholesterolemic effect of Lactobacillus reuteri in hypercholesterolemic mice. J Dairy Sci 1998, 81(9):2336-2340.

79. Goldin BR, Gualtieri L, Moore RP: The effect of Lactobacillus GG on the initiation and promotion of DMH-induced intestinal tumors in the rat. 1996.

80. Vuyst L, Vandamme EJ: Bacteriocins of lactic acid bacteria: microbiology, genetics and applications: Springer US; 1994.

81. Aso Y, Akazan H: Prophylactic effect of a Lactobacillus casei preparation on the recurrence of superficial bladder cancer. Urologia internationalis 1992, 49(3):125-129.

82. Mallett A, Bearne C, Rowland I: The influence of incubation pH on the activity of rat and human gut flora enzymes. Journal of applied bacteriology 1989, 66(5):433-437.

83. Gorbach S, Chang T-W, Goldin B: Successful treatment of relapsing Clostridium difficile colitis with Lactobacillus GG. The Lancet 1987, 330(8574):1519.

84. Lilly DM, Stillwell RH: Probiotics: growth-promoting factors produced by microorganisms. Science (New York, NY) 1965, 147(3659):747-748. 
Chapter 2 
Potential contribution of cereal and milk based fermented foods to dietary nutrient intake of 1-5 years old children in Central province in Zambia.

Justin Chileshe ${ }^{1,2,3}$, Elise F Talsma ${ }^{2}$, Sijmen E Schoustra ${ }^{1,4}$, Karin J Borgonjen-van den Berg ${ }^{2}$, Ray Handema $^{3}$, Bas J Zwaan ${ }^{1}$ and Inge D Brouwer ${ }^{2}$

${ }^{1}$ Plant Sciences Group, Laboratory of Genetics, Wageningen University and Research, the Netherlands

${ }^{2}$ Division of Human Nutrition and Health, Wageningen University and Research, the Netherlands

${ }^{3}$ Tropical Diseases Research Centre, Ndola, Zambia

${ }^{4}$ University of Zambia, Department of Food Science and Nutrition, School of Agricultural Sciences, Zambia 


\section{Abstract}

Zambia is still facing undernutrition and micronutrient deficiencies despite fortification and supplementation programmes stressing the need for additional solutions. Fermented foods have the potential to improve nutrient intake and, therefore, could have an important role in food based recommendations (FBRs) to ensure adequate intake of nutrients for optimal health of populations. Secondary dietary intake data was used in Optifood, a linear programming software to develop FBRs, for children aged 1-3 and 4-5 years in Mkushi district of Zambia. Three scenarios per age group were modeled to determine FBRs based on: (1) local available foods (FBR) (2) FBR and Mabisi, a fermented milk beverage, and (3) FBR with Munkoyo, a cereal fermented beverage. The scenarios were compared to assess whether addition of Mabisi or Munkoyo achieved a better nutrient intake. FBRs based on only locally available non-fermented foods did not meet $\geq 70 \%$ of recommended nutrient intake (RNI) for calcium, fat, iron and zinc, so-called problem nutrients. The addition of Munkoyo to the FBRs did not reduce the number of problem nutrients, but after adding Mabisi to the FBR's only iron (67\% of RNI) in the 1-3 year age group and only zinc (67\% of RNI) in the $4-5$ year age group remained problem nutrients. Mabisi, a fermented milk product in combination with the local food pattern is a good additional source of nutrients for these age groups. However, additional nutrition sensitive and cost-effective measures would still be needed to improve nutrient intake, especially that of iron and zinc.

\section{Introduction}

Undernutrition remains a severe public health problem in Zambia where $40 \%$ of the children under the age of five years are stunted, $15 \%$ have underweight and $6 \%$ are wasted [1]. Undernutrition in children leads to reduced growth, cognitive development impairment, greater susceptibility to infections and higher risk of mortality $[2,3]$. Micronutrient deficiencies are also high in Zambia with $60 \%$ of the children between 6-59 months old suffering from anaemia which may result from iron deficiency among others and $54 \%$ of them affected by vitamin A deficiency [4-6]. Different strategies and interventions exist to prevent or reduce undernutrition and micronutrient deficiencies. These include food-based strategies such as dietary diversification and food fortification, as well as nutrition education, public health and food safety measures, and supplementation [7]. Fortification and supplementation of micronutrients are seen as short and medium term solutions but are more expensive, and adherence might be low if intensive stimulation programs are not put in place [8]. Despite sugar and salt fortification and supplementation implemented to reduce micronutrient deficiencies for more than two decades, Zambia is still facing undernutrition and micronutrient deficiencies stressing the need for additional solutions [9]. 
In Zambia there are a number of nutritionally dense foods locally available that can potentially be utilised to alleviate these nutrition problems [10]. The formulation, testing and implementation of food based recommendations (FBRs) can contribute to improve the nutrient intake, especially when these local nutrient dense foods are incorporated [11]. Such FBRs facilitate the formulation of Infant and Young Child Feeding (IYCF) practices being identified as one of the most effective public health interventions to improve young child survival in developing countries [12]. Especially in young children, the composition of diets and quality of foods consumed by populations have a direct impact on their health and wellbeing and thus FBRs are important in providing population-level and context specific guidance on consumption of appropriate foods to meet their nutritional needs [12].

Optifood is a linear programming software that allows formulation of FBRs based on the best combinations of local foods to optimize nutrient intake and model the extent to which these can supply nutritionally adequate diets [13-17]. In previous studies conducted with intake data from children of 6 months to 23 months old, gaps in intake of specific nutrients have been found especially that of calcium, iron and zinc [15-18]. Adding recommendations on increase of intake of animal source foods can potentially improve intake of calcium, iron and zinc but consumption of such foods in Zambia is low where diets are predominantly plant-based. In plant-based diets, preparation methods such as fermentation can be a method to make nutrients more bioavailable [19]. Phytates are present as storage compounds of phosphorous in cereals as complexes with metal cations such as iron, zinc and calcium. Phytates can be degraded by fermentation, in which microbial activity lowers the $\mathrm{pH}$ providing an optimum environment for enzymatic degradation by phytase leading to an increase in the cations making them bioavailable [20]. Fermentation is also beneficial in dairy products, as this process helps to convert lactose into digestible components hence making milk more tolerable [21]. More generally, fermentation also gives food longer shelf life and contributes to a healthy ecology of intestinal bacteria which promotes general health, through fermenting bacteria (usually lactic acid bacteria) that serve as probiotics [19, 22-25]. Fermented foods traditionally are an important part of the diet in many countries and are now being advocated for inclusion in food based recommendations for regular consumption in some countries [26]. Zambia has a wide range of local fermented foods similar to western yoghurt, wine, and beer [27]. A number of traditional non-alcoholic fermented beverages are available such as Mabisi and Munkoyo which are consumed by all age groups [28]. Munkoyo is a fermented beverage made from maize porridge with Rhynchosia venulosa (Munkoyo) roots added whereas Mabisi is a fermented milk product made by allowing raw milk to ferment at ambient temperature in containers such as buckets $[28,29]$.

This study used linear programming to explore secondary dietary intake data of children, 1-5 years of age, in Mkushi, Zambia to assess the potential effect on nutrient adequacy of adding traditional fermented foods (Mabisi and Munkoyo) into FBRs. 


\section{Methods}

Study Design

This study was based on cross-sectional dietary intake data collected previously with children in a rural, maize consuming population in Mkushi (Central Province, Zambia) [30]. Data was collected during harvest/early post-harvest season (May-June 2009) using the 24-hour recall method and was used to model weekly food based recommendations for children aged 1-5 years, with and without inclusion of Mabisi or Munkoyo.

The initial study was approved by the Tropical Diseases Research Centre (TDRC) Ethics review committee (Ndola, Zambia) reference number TDRC/ERC/0705/0409 and the International Food Policy Research Institute (Washington, DC, USA) Institutional review board [30].

\section{Subjects}

The original study assessed dietary intake of 320 children in Mkushi [30]. From this data set we selected two groups of non-breastfed children aged 1-3 years $(n=156)$ and 4-5 years $(n=65)$ for Optifood programming, based on the different recommended nutrient intakes of these two age groups. Breastfed children were excluded because of a too small sample size $(n=15)$.

\section{Dietary intake assessment}

The dietary intake data was collected using the multi pass 24 hour recall as described elsewhere [31]. To collect the dietary intake data [30], the mothers of the children were asked to recall all foods and beverages consumed by their children, including amounts, during 24-hours of the previous day. Food portion sizes were estimated using examples of real foods, scaled photographs, or in volumes using standardized measuring spoons and cylinders carried by interviewers, and calibrated modelling clay. Information on feeding habits including plate sharing during meal times by children or family members, and on foods and quantities consumed outside of the home was collected. To convert portion sizes recorded in volumes to gram weight equivalents, a local conversion table was developed. Grams of ingredients consumed from the composite dishes was derived from the recipe data collected during the recall study or from standard recipes compiled prior to the initial study. Nutrient intake calculations were based on the food composition tables (FCT) developed by the initial study[30] [31].

\section{Data preparation}

Data from the 24 hour recalls was used to generate model parameters for Optifood using Excel and Access 2010 (Microsoft Corporation) [32]. Model parameters per age group were defined as follows: (I) A list of non-condiment foods and drinks consumed by $\geq 5 \%$ of the children during the recall period 
to ensure the commonly consumed foods are included; (II) The serving size of each food was defined as the medium serving size in grams per day for all children who consumed that particular food; (III) The minimum and maximum number of servings per week for each food group and food subgroup defined as the $10^{\text {th }}$ and $90^{\text {th }}$ percentiles of the serving counts respectively. The maximum and minimum number of servings per food within a food subgroup was estimated based on percentage of children who consumed the food $[16,17,33,34]$. In the initial study only 2 children in the $4-5$ age group were reported to have consumed Mabisi with the volume similar to that of tea consumed and thus the serving size/day for Mabisi in the two age groups were estimated based on the tea serving volume/day; (IV) Staple foods were identified as foods belonging to the food groups' grain and grain products or starchy roots. Snacks were defined as foods consumed only in between meals. The type of meal (snack or staple) was determined based on the nature of the food and time of the food consumption.

Thirteen nutrients were selected for analysis of nutrient adequacy including total fat, total protein, calcium, vitamin $C$, thiamin, riboflavin, niacin, vitamin $B 6$, folate, vitamin $B 12$, vitamin $A$ as $R A E$, iron and zinc using the FAO/WHO RNIs [35].

Energy constraints were used to model FBR's that ensured average energy requirement for the two age groups, by using mean body weight obtained from the initial study for each age group [36]. Dietary patterns in Zambia like in other developing countries are often rich in plant based foods and are high in Phytates, thus low bioavailability of iron (RNI: $11.6 \mathrm{mg} /$ day assuming $5 \%$ bioavailability) and zinc (RNI: $8.3 \mathrm{mg} /$ day assuming $15 \%$ bioavailability) were taken into account [37, 38].

The FCT used for this study included nutrient values for Mabisi (FCT as Sour milk). Nutrient values for Munkoyo were based on a composited recipe consisting of maize meal and water. The World Health Organization (WHO) estimated that the maximum iron and zinc bioavailability in maize based diets ranges between 10 and 15\% [39]. Fermentation of maize based foods or beverages improves bioavailability of iron and zinc mainly by the breakdown of Phytates and is estimated to result in a $5 \%$ increase in bioavailability for iron, but not clear for zinc [40]. The values of iron and zinc for munkoyo in the FCT were therefore increased by $5 \%$ to $10 \%$ and $20 \%$ respectively to take into account the increase in bioavailability through fermentation.

\section{Analysis in Optifood}

All analyses were carried out with Optifood program version 4.0.9.0 using a three module approach, based on linear programming to design population specific weekly FBRs. Per target group, three scenarios were modelled to develop FBR, namely: a) local foods without Munkoyo or Mabisi, b) local foods with Munkoyo included and c) local foods with Mabisi included. The linear programming analyses used in this study have been described in detail by others [11, 16, 33, 34]. In summary, for each scenario in each target group data was checked by running module 1 (to set up model parameters) to 
ensure that model parameters were producing realistic diets with energy contents within a sufficient range to allow for modelling modeling. An expert who was familiar with local dietary patterns then examined the foods selected in these 16 optimized 7-day diets to decide whether at least some individuals from the population could consume them.

Module 2 (to identify food based recommendations) was run for each of the three scenarios and for each age group to develop two optimized diets called the food pattern diet and no-food pattern diet. The food pattern diet is the best diet with minimized deviations from RNIs within the target population's average food pattern. The no-food pattern diet is the best diet with minimized deviations from RNIs deviating from the average food pattern while remaining within lower and upper food group constraints. Module 2 was used to identify the nutrient dense food (sub) groups that were likely to improve nutrient adequacy and needed to be tested in module 3. Foods that contributed for at least $5 \%$ to any of the nutrients were identified as nutrient dense foods. [17, 33, 34].

In Module 3 (to test alternative sets of FBRs to select the best dietary recommendations for the target population) diets were modelled for all three scenarios in both target groups, two 7-day diets per nutrient (i.e. in total 26 diets) were modelled of which 13 maximised (best-case scenario) and 13 minimized the nutrient content of the diet, for one nutrient (worst-case scenario), by preferentially selecting respectively the lowest and highest nutrient dense foods for that specific nutrient.

In step (i) of module 3, a no recommendation diet was run for each scenario to identify problem nutrients. Problem nutrients were defined as nutrients that were less than $100 \%$ of RNI in the bestcase scenario (maximized diet) of module 3 diet modelled without FBR constraints $[17,33,34]$.

In step (ii) of module 3, food groups with weekly servings above zero obtained in module 2 best food pattern diet, and nutrient dense foods and their accompanying food (sub) groups identified in module 2 were tested individually and were combined in step (iii) of module 3, the final FBRs per scenario in each target group was selected based on the combination of foods and food (sub) groups that covered $70 \%$ of the RNI in the worst case scenario for most nutrients, minimizing the deviation from the local food pattern as much as possible. The final FBRs were compared between the three scenarios.

\section{Results}

Data of 156 children in the 1-3 year age group with an average age of 2.8 years and 65 children in the 4-5 year age group with an average age of 4.3 years were used in this study. Girls represented $47 \%$ $(n=74)$ in $1-3$ age group and $61 \%(n=45)$ in $4-5$ age group.

A total of 164 foods (with 28 foods consumed by $\geq 5 \%$ of children) were consumed by children in $1-3$ year group and 115 foods (with 31 foods consumed by $\geq 5 \%$ of children) were consumed by children in the 4-5 year group. The most commonly consumed foods in both age groups included vegetable oil, onions, tomatoes, maize flour, and rape leaves as a pro-vitamin A rich source green leafy vegetable. 
Maize meal (medium serving per day was $242 \mathrm{~g}$ for $1-3$ year olds and $255 \mathrm{~g}$ for $4-5$ year olds) was consumed in high amounts compared to other foods. The smallest serving sizes were for white sugar (14g/day) for the 1-3 year olds and for brown sugar (20g/day) for the 4-5 year olds. The consumption frequencies of the foods varied from 0 to 14 times per week. An overview of all the foods, their corresponding serving sizes and consumption frequencies used for analysis is shown in Table 1 for each of the two age groups.

\section{Linear programming}

Diets produced in Module 1 (to set up model parameters) were feasible and no changes in model parameters were needed. The number of nutrients reaching $\geq 100 \%$ of RNI in the two best diets of the three scenarios for each age group as analysed in module 2 (to identify food based recommendations), are shown in Table 2. For 1-3 years old children, 8 nutrients for the average food pattern and 10 nutrients for best food pattern reached $\geq 100 \%$, with no differences whether in- or excluding Mabisi or Munkoyo. For 4-5 years old children, the average food pattern diet covered $>100 \%$ RNI for 5 nutrients whether or not including Mabisi, however, including Munkoyo increased number of nutrients covering $\geq 100 \%$ RNI to 6 . For this age group, 11 nutrients reached $\geq 100 \%$ RNI for the best food pattern without differences with or without Mabisi or Munkoyo. Fat, calcium and zinc were identified as problem nutrients for this age group. In module 2, food groups that included grains \& grain products and vegetables; food sub groups that included nuts/seeds \& unsweetened products, cooked beans/lentils/peas and small whole fish with bones; and rape leaves as a single food were identified as nutrient-dense foods to be included to develop food based recommendations in module 3 (to test alternative sets of FBRs to select the best dietary recommendations for the target population).

In module 3 (i), fat, calcium, iron and zinc were identified as problem nutrients (reaching $<100 \%$ RNI) in the best case scenario diet for the 1-3 year old group and fat, calcium and zinc for the 4-5 year old group. Testing food (sub) groups identified as nutrient dense in module 2, individually and combined (in module 3ii and iii), resulted in an increased coverage of the problem nutrients but none reached $\geq 70 \% \mathrm{RNI}$, and, hence, remained as problem nutrients (Table 3). Testing with inclusion of Munkoyo did not change these results (Table 4), however, inclusion of Mabisi resulted in fat, calcium and zinc disappearing as problem nutrient as their intake reached $\geq 70 \%$ RNI. Only iron remained as problem nutrient (Table 5).

Fat, calcium and zinc were identified as problem nutrients (reaching $<100 \% \mathrm{RNI}$ ) in the best-case scenario diet for the 4-5 year olds and, when testing food (sub) groups identified as nutrient dense in module 2 , individually and combined (in module $3 \mathrm{~b}$ and $\mathrm{c}$ ), these nutrients remained as problem nutrients irrespective whether or not Munkoyo was included (Table 6 and 7). However, including 
Mabisi showed improvement in calcium ( $\geq 70 \% \mathrm{RNI}$ ) with fat and zinc remaining below $70 \%$ RNI (Table 8).

The final recommendations (with most nutrients reaching $\geq 70 \% \mathrm{RNI}$ in the worst case scenario of the FBRs) for the 1-3year age group included the following serves per week: Vegetables 21 servings; Mabisi 7 servings; Grains and grain products 7 servings; Cooked beans, lentils and peas 3 servings; Nuts, seeds and unsweetened products 4 servings; and Rape leaves 7 servings; Small whole fish with bones 2 servings (table 5).

The final recommendations (with most nutrients reaching $\geq 70 \%$ RNI in the worst case scenario of the FBRs) for 4-5 year age group included the following servings per week: Vegetables 21 servings; Mabisi 6 servings; grains and grain products 6 servings; Cooked beans, lentils and peas 3 servings; Nuts, seeds and unsweetened products 5 servings; and Rape leaves 7 servings; Small whole fish with bones 2 servings (table 8 ).

\section{Discussion}

The current analysis revealed that nutrient intake among children aged 1-5 years in Zambia can be profoundly improved through carefully selected combinations of locally available foods but will not be able to cover the nutrient requirements for fat, calcium, iron and zinc. Only the inclusion of Mabisi (fermented milk) and not Munkoyo can sufficiently improve fat, calcium, iron (for 1-3 years olds only) and zinc (for 4-5 years olds only) intake for 1-5 year olds as indicated in Figure 1.

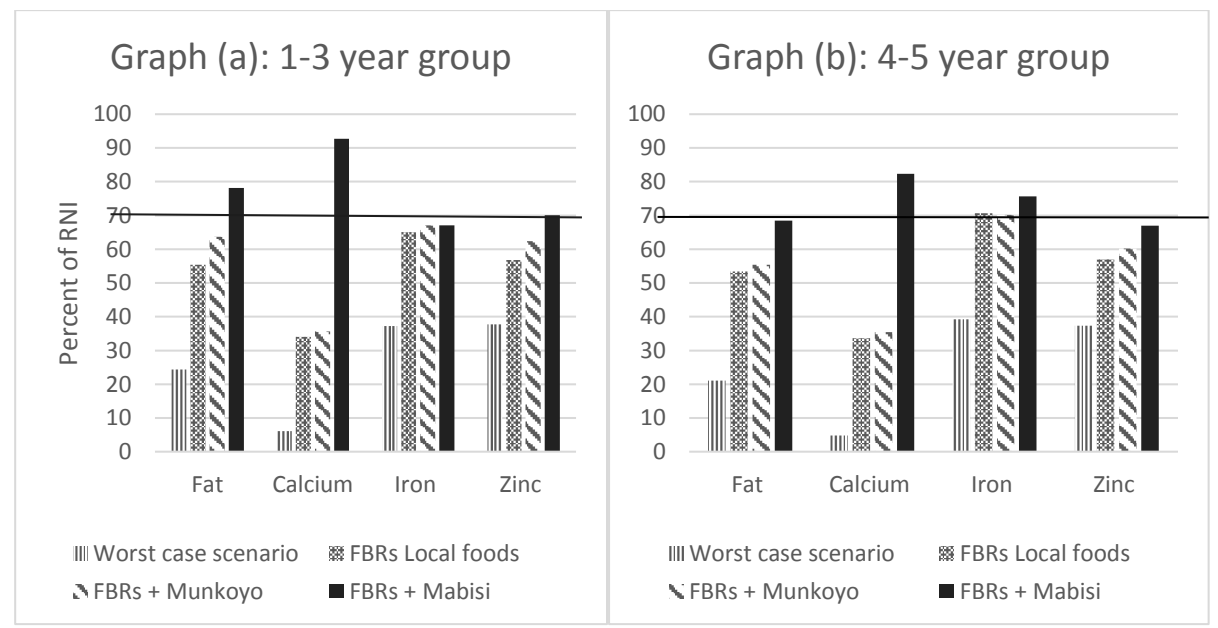

Figure 1: Comparison of problem nutrients for the three scenarios (FBRs with local foods only, FBRs with Munkoyo added and FBRs with Mabisi added) against the worst case scenarios for the two age groups, 1-3 years old in graph (a) and 4-5 years old in graph (b). 
Notes: The $\mathrm{y}$-axis shows the \% of RNI and the $\mathrm{x}$-axis shows the problem nutrients. RNI is Recommended Nutrient Intake; FBRs is Food Based Recommendations. These are the nutrients that did not reach 70\% RNI when diets were modelled for the 3 scenarios compared with the worst-case-scenario. The rest of the nutrients selected and modelled in Optifood are not depicted in this figure because they were all $\geq 70 \%$ RNI for all the scenarios. In the figure, the diet with Mabisi recorded the highest increase compared to the worst-case-scenario for the problem nutrients in both age groups.

Results from our study indicate that among 1-5 year old children in Mkushi, the intake of fat, iron, zinc and calcium is below the requirements. The identified problem nutrients in our study are consistent with other findings in Zambia. Calcium shows the lowest achievable \% of RNI in the scenario's with and without Munkoyo added. In Mkushi, another study also found nutrient adequacy for children between 4 and 8 years old for most nutrients except for calcium [41]. Another study in Zambia found that children between 6-18 months had inadequate nutrient intakes for iron, zinc and calcium [42]. Low consumption of animal source foods could partly explain low intake of fat, calcium, iron and zinc nutrients in our population due to a substantial increase in production and consumption of cassava, maize and vegetable oils and a corresponding decrease in that of animal source foods observed in the past decades [9]. Inclusion of Mabisi in the food based dietary recommendations increased the potential of adequate intake of fat, calcium, zinc and iron in our study. A review on the impact of lipidbased nutrient supplements (LNS) plus complementary foods on health, nutrition and developmental outcomes among infants and young children [43] suggested that LNS plus complementary feeding compared to no intervention is effective at improving growth outcomes and anaemia among children aged 6 to 23 months in low- and middle-income countries (LMIC) in Asia and Africa, and more effective if provided over a longer duration of time (over 12 months). A trial in Malawi comparing the developmental outcomes of 18-month-old infants who received complementary feeding for 1 year either with lipid-based nutrient supplements or micronutrient fortified corn-soy porridge [44] found that the two types of interventions have comparable developmental outcomes by 18 months of age. A number of other trials conducted on yogurt (a fermented milk product with Lactobacillus bulgaricus and Streptococcus thermophilus) consumption have linked this yogurt intake to better health outcomes including weight management, type 2 diabetes, cardiovascular, disease risk, bone health, gastrointestinal (GI) health, malnutrition, immunological parameters and overall mortality [44-52]. In many of these studies microbial diversity appeared to increase in subjects consuming yogurt and the association between a greater microbial diversity and better health conditions has been attributed to yogurt consumption. Since Mabisi is a fermented milk product with the potential to contribute to adequate intake of fat, calcium and zinc for 1-3 year aged children and fat, calcium and iron for 4-5 year aged children, regular consumption could contribute to the improvement in nutritional status, development functions, and gut function through fermenting bacteria. 
Evidence has been building up over the recent years concerning the importance of the microbiome to our general health with arguably diet being the most important factor in shaping it [53]. Overall, fermented foods have been found to shift the microbial composition in the gut microbiome toward higher abundance of lactic acid bacteria being beneficial for general health of individuals $[54,55]$. Therefore, the increase in nutrient intake observed in the FBRs with Mabisi would be complemented by the beneficial effects of the microbes, as fermented foods may contain live microorganisms that may improve gastrointestinal health and provide other health benefits [56]. Munkoyo (a cereal based fermented product) did not confer significant benefits in improving nutrient intake but the children may benefit from the fermenting microbes - that include high abundance of lactic acid bacteria - in this product. Chilton et al, in a review promoting inclusion of fermented foods in dietary guidelines established that the extensive use of and nutritional and health benefits derived from the fermented foods are evident enough for recommendation of regular consumption [26].

The feasibility and generalizability of identified food based dietary recommendations need further attention and it should be evaluated before implementation; for instance whether the required behaviour change is feasible [33]. Further, the foods included in the food based dietary guidelines should be available in sufficient amounts, although availability of foods does not always result in increased consumption as other aspects influence consumption, for example accessibility and affordability [57]. Cost of the locally available foods was not taken into account when modelling the different scenario diets. While Mabisi is generally considered a low-cost food, it is not known whether the final FBRs (including Mabisi) are affordable for the parents or caretakers of the children. Generally socio-economic factors influence food choices and nutrient dense foods are in general more expensive than low nutrient dense foods $[58,59]$. Furthermore, Mabisi should be acceptable for consumption. From our dietary intake it appeared that Mabisi was consumed by only three children (one from the 1-3 year age group and 2 from the 4-5 year age group). However other studies about traditional fermented foods in Zambia [28] have shown that these foods are consumed on a regular basis in all age groups and are culturally accepted. Lastly, data from only one district in Zambia (Mkushi) was used. Although generally in Zambia similar dietary patterns are observed throughout the country with main foods being cereals, roots \& tubers and vegetables [60], some foods in our FBRs may not be available in all areas of Zambia. Therefore use of data from a national representative sample would probably result in FBRs that are generalizable for the whole country.

There are some limitations in this study that are acknowledged. First, we acknowledge that recall bias in dietary intake data collection using 24 hour recall is inevitable. Precautions were taken through well trained interviewers, the use of a food frequency questionnaire (FFQ) to determine the frequency of specific food items, proper calibrations and the use of pictures and plastic bowls to better visualize and estimate the serving sizes. Often in recalls, nutrition dense foods that are irregularly consumed and 
snacks and fruits are often forgotten. Nevertheless, we could not avoid introducing errors in the data which could have affected our results, but we tried to keep it to a minimum.

Second, FBRs were developed for children between 1 and 5 years who are not breastfed due to very few children being breastfed after 1 year of age. The World Health Organization and UNICEF recommends initiation of breastfeeding within the first hour after the birth, exclusive breastfeeding for the first six months and continued breastfeeding for two years or more, together with safe, nutritionally adequate, age appropriate, responsive complementary feeding starting at the age of sixth months [6]. Part of the target group in this study falls in this particular age range. If breastfeeding were included in the modelling for the 1-2 years old, probably the effect of Mabisi would have been less as the breastmilk would have provided for some of the problem nutrients. However, breastfeeding after 1 year was very low, accounting for less than $5 \%$ children, and was far lower than that of the national average of $92 \%$ and $42 \%$ of all children still breastfed at age 1 year and at 2 years respectively [1]. It may be that in this region of the country breastfeeding after age 1 year is low and that changing behaviour towards extending the breastfeeding period to 2 years or beyond may be challenging due to socio-cultural issues. We think that inclusion of Mabisi is more acceptable than extension of breastfeeding and that also breastmilk lacks the beneficial effects of fermentation, however it is acknowledged that Mabisi may have a higher safety risk.

Third, we used a combination of food composition tables (FCTs) to develop the one used in this study and this may have introduced errors due to nutrient variations introduced. We did not have the nutrient composition of Mabisi and used the composition of sour milk that is considered similar to Mabisi from the FCT. We do not expect any significant differences in nutrients between Mabisi and sour milk as they are similar products made in the same way but with different names in different regions of the country. It is clear that fermentation reduces phytates and increases bioavailability of minerals such iron and zinc in foods and that information on iron is available in literature but it is unclear for zinc [40]. We increased zinc values modestly by $5 \%$, similar to that of iron in the FCT taking into account the reduction of phytate that has been shown before of $15-46 \%$ after maize porridge fermentation [61] and that the increase in iron bioavailability positively correlated with zinc amounts determined from in vitro studies [62]. It is remarkable that dried small whole fish species in our FCT contained very high vitamin $B 12$ values reaching $\geq 300 \%$ of the $\mathrm{RNI}$ for vitamin B12. These values were based on data published in the African Journal of Food, Agriculture, Nutrition and Development in 2010 and a report published by Nyirenda et al in 2007 [10, 63]. In these studies vitamin B12 values were similar to the FCT values used in this study for dried small whole fish species. It is known that dietary patterns in low and middle income countries are mostly plant based and as animal source products are rich sources of vitamin B12, it would be expected that deficiencies in vitamin B12 exist. In a study that assessed vitamin B12 status among Zambian children under the age of five years, high levels of vitamin 
B12 deficiencies were found (87\%) [64]. The high values for vitamin B12 in fish in our FCT might have led to an overestimation of the dietary intake of vitamin B12 for the target group. To improve the data, a direct chemical analysis of the fish consumed would have given better estimates of vitamin B12 intake.

\section{Conclusions}

This study shows that FBRs for Zambian non-breastfed, 1-5 year old children based on only locally available foods into FBRs do not meet the required intakes for fat, calcium, iron and zinc and that the inclusion of Mabisi (and not Munkoyo) can have a major impact on the nutrient adequacy. Results in this study indicates the importance of the inclusion of Mabisi, a fermented milk product in the local food diet as a good additional source of nutrients for these age groups. However, to improve iron (for 1-3 years old) and zinc (for 4-5 years old) intake, alternative strategies should be found. The results from this analysis can serve as a guide for designing evidence based dietary recommendations under local conditions. Furthermore, additional data on for example cost of foods would still be needed to improve feasibility of developed FBRs. 
1. Central Statistical Office/Zambia, Ministry of Health/Zambia, University of Zambia Teaching Hospital Virology Laboratory, University of Zambia Department of Population Studies, Tropical Diseases Research Centre/Zambia, ICF International: Zambia Demographic and Health Survey 2013-14. In. Rockville, Maryland, USA: Central Statistical Office/Zambia, Ministry of Health/Zambia, and ICF International; 2015.

2. Reinhardt K, Fanzo J: Addressing Chronic Malnutrition through Multi-Sectoral, Sustainable Approaches: A Review of the Causes and Consequences. Frontiers in Nutrition 2014, 1:13.

3. Walker SP, Chang SM, Powell CA, Simonoff E, Grantham-McGregor SM: Early childhood stunting is associated with poor psychological functioning in late adolescence and effects are reduced by psychosocial stimulation. The Journal of nutrition 2007, 137(11):2464-2469.

4. Zambia MoH: Zambia national malaria indicator survey 2015. In. Lusaka; 2015.

5. Food N, Nutrition Commission of Zambia UoZ, MOST: the USAID Micronutrient Program, United States Center for Disease Control: Report of the national survey to evaluate the impact of vitamin A interventions in Zambia in July and November 2003. In.: NFNC Lusaka; 2004.

6. WHO U: UNU. Iron deficiency anaemia: assessment, prevention, and control. Geneva, WHO 2001.

7. Burchi F, Fanzo J, Frison E: The Role of Food and Nutrition System Approaches in Tackling Hidden Hunger. International Journal of Environmental Research and Public Health 2011, 8(2):358.

8. Mildon A, Klaas N, O'Leary M, Yiannakis M: Can fortification be implemented in rural African communities where micronutrient deficiencies are greatest? Lessons from projects in Malawi, Tanzania, and Senegal. Food and Nutrition Bulletin 2015, 36(1):3-13.

9. Zhang Z, Goldsmith PD, Winter-Nelson A: The Importance of Animal Source Foods for Nutrient Sufficiency in the Developing World:The Zambia Scenario. Food and Nutrition Bulletin 2016, 37(3):303-316.

10. Nyirenda DB, Musukwa M, Mugode RH: The common Zambian foodstuff, ethnicity, preparation and nutrient composition of selected foods. Zambia: Child Health Project of the Ministry of Health, and Boston, USA: Boston University 2007.

11. Ferguson EL, Darmon N, Briend A, Premachandra IM: Food-based dietary guidelines can be developed and tested using linear programming analysis. The Journal of nutrition 2004, 134(4):951-957.

12. Bhutta ZA, Das JK, Rizvi A, Gaffey MF, Walker N, Horton S, Webb P, Lartey A, Black RE: Evidence-based interventions for improvement of maternal and child nutrition: what can be done and at what cost? The Lancet 2013, 382(9890):452-477.

13. Daelmans B, Ferguson E, Lutter CK, Singh N, Pachón H, Creed-Kanashiro H, Woldt M, Mangasaryan N, Cheung E, Mir R et al: Designing appropriate complementary feeding recommendations: tools for programmatic action. Maternal \& Child Nutrition 2013, 9(S2):116-130.

14. Levesque S, Delisle H, Agueh V: Contribution to the development of a food guide in Benin: linear programming for the optimization of local diets. Public health nutrition 2015, 18(04):622-631.

15. Santika O, Fahmida U, Ferguson EL: Development of Food-Based Complementary Feeding Recommendations for 9- to 11-Month-Old Peri-Urban Indonesian Infants Using Linear Programming. The Journal of nutrition 2009, 139(1):135-141.

16. Skau JK, Bunthang T, Chamnan C, Wieringa FT, Dijkhuizen MA, Roos N, Ferguson EL: The use of linear programming to determine whether a formulated complementary food product can ensure adequate nutrients for 6- to 11-month-old Cambodian infants. The American Journal of Clinical Nutrition 2014. 
17. Vossenaar M, Knight FA, Tumilowicz A, Hotz C, Chege P, Ferguson EL: Context-specific complementary feeding recommendations developed using Optifood could improve the diets of breast-fed infants and young children from diverse livelihood groups in northern Kenya. Public health nutrition 2017, 20(6):971-983.

18. Ferguson E, Chege P, Kimiywe J, Wiesmann D, Hotz CCMCNOAR: Zinc, iron and calcium are major limiting nutrients in the complementary diets of rural Kenyan children. Maternal \& Child Nutrition 2015, 11:6-20.

19. Hotz C, Gibson RS: Traditional food-processing and preparation practices to enhance the bioavailability of micronutrients in plant-based diets. The Journal of nutrition 2007, 137(4):1097-1100.

20. Gupta RK, Gangoliya SS, Singh NK: Reduction of phytic acid and enhancement of bioavailable micronutrients in food grains. Journal of food science and technology 2015, 52(2):676-684.

21. de Vrese M, Stegelmann A, Richter B, Fenselau S, Laue C, Schrezenmeir J: Probiotics-compensation for lactase insufficiency. Am J Clin Nutr 2001, 73(2 Suppl):421s-429s.

22. Egounlety M, Aworh OC, Akingbala JO, Houben JH, Nago MC: Nutritional and sensory evaluation of tempe-fortified maize-based weaning foods. Int J Food Sci Nutr 2002, 53(1):15-27.

23. Kort R, Sybesma W: Probiotics for every body. Trends in biotechnology 2012, 30(12):613615.

24. Singh RK, Chang H-W, Yan D, Lee KM, Ucmak D, Wong K, Abrouk M, Farahnik B, Nakamura M, Zhu TH et al: Influence of diet on the gut microbiome and implications for human health. Journal of Translational Medicine 2017, 15(1):73.

25. Zimmermann MB, Chassard C, Rohner F, N'Goran E K, Nindjin C, Dostal A, Utzinger J, Ghattas $\mathrm{H}$, Lacroix $\mathrm{C}$, Hurrell RF: The effects of iron fortification on the gut microbiota in African children: a randomized controlled trial in Cote d'Ivoire. Am J Clin Nutr 2010, 92(6):14061415.

26. Chilton SN, Burton JP, Reid G: Inclusion of Fermented Foods in Food Guides around the World. Nutrients 2015, 7(1):390-404.

27. Nguz K, Kasase C, Phiri C, Shindano J, Hikeezi D: Isolation and inhibitory effects of lactic acid bacteria from selected traditional fermented beverages from rural Zambia on some food pathogens. Journal of Science and Technology (Lusaka, Zambia) 2004(special ed.):9-16.

28. Schoustra SE, Kasase C, Toarta C, Kassen R, Poulain AJ: Microbial Community Structure of Three Traditional Zambian Fermented Products: Mabisi, Chibwantu and Munkoyo. PloS one 2013, 8(5):e63948.

29. Moonga HB, Schoustra SE, Linnemann AR, Kuntashula E, Shindano J, Smid EJ: The art of mabisi production: A traditional fermented milk. PloS one 2019, 14(3):e0213541.

30. Hotz C, Chileshe J, Siamusantu W, Palaniappan U, Kafwembe E: Vitamin A intake and infection are associated with plasma retinol among pre-school children in rural Zambia. Public Health Nutr 2012, 15(9):1688-1696.

31. Hotz C, Chileshe, J., Siamusantu, W. and Palaniappan, U: Nutrition Survey in Central and Eastern Provinces , Zambia. In.; 2009.

32. Ferguson EL, Darmon N, Fahmida U, Fitriyanti S, Harper TB, Premachandra IM: Design of optimal food-based complementary feeding recommendations and identification of key "problem nutrients" using goal programming. The Journal of nutrition 2006, 136(9):23992404.

33. Talsma EF, Borgonjen-van den Berg KJ, Melse-Boonstra A, Mayer EV, Verhoef H, Demir AY, Ferguson EL, Kok FJ, Brouwer ID: The potential contribution of yellow cassava to dietary nutrient adequacy of primary-school children in Eastern Kenya; the use of linear programming. Public health nutrition 2018, 21(2):365-376. 
34. Kujinga $P$, Borgonjen-van den Berg KJ, Superchi C, ten Hove HJ, Onyango EO, Andang'o $P$, Galetti V, Zimmerman MB, Moretti D, Brouwer ID: Combining food-based dietary recommendations using Optifood with zinc-fortified water potentially improves nutrient adequacy among 4-to 6-year-old children in Kisumu West district, Kenya. Maternal \& Child Nutrition 2018, 14(2):e12515.

35. Joint FWU: Human energy requirements. Report of a Joint FAO/WHO/UNU Expert Consultation, Rome, 17-24 October 2001. 2004.

36. Group WHOMGRS: WHO Child Growth Standards based on length/height, weight and age. Acta Paediatr Suppl 2006, 450:76-85.

37. Gibson RS, Perlas L, Hotz C: Improving the bioavailability of nutrients in plant foods at the household level. Proceedings of the Nutrition Society 2006, 65(02):160-168.

38. Hurrell RF, Reddy MB, Juillerat M-A, Cook JD: Degradation of phytic acid in cereal porridges improves iron absorption by human subjects. The American Journal of Clinical Nutrition 2003, 77(5):1213-1219.

39. Staff WHO, Organization WH, UNICEF.: Global strategy for infant and young child feeding: World Health Organization; 2003.

40. Suri DJ, Tanumihardjo SA: Effects of Different Processing Methods on the Micronutrient and Phytochemical Contents of Maize: From A to Z. Comprehensive Reviews in Food Science and Food Safety 2016, 15(5):912-926.

41. Caswell BL, Talegawkar SA, Dyer B, Siamusantu W, Klemm RD, Palmer AC: Assessing child nutrient intakes using a tablet-based 24-hour recall tool in rural Zambia. Food and nutrition bulletin 2015, 36(4):467-480.

42. Owino V, Amadi B, Sinkala M, Filteau S, Tomkins A: Complementary feeding practices and nutrient intake from habitual complementary foods of infants and children aged 6-18 months old in Lusaka, Zambia. African Journal of Food, Agriculture, Nutrition and Development 2008, 8(1):28-47.

43. Das JK, Salam RA, Hadi YB, Sheikh SS, Bhutta AZ, Prinzo ZW, Bhutta ZA: Preventive lipidbased nutrient supplements given with complementary foods to infants and young children 6 to 23 months of age for health, nutrition, and developmental outcomes. Cochrane Database of Systematic Reviews 2019(5).

44. Phuka JC, Gladstone M, Maleta K, Thakwalakwa C, Cheung YB, Briend A, Manary MJ, Ashorn $P$ : Developmental outcomes among 18-month-old Malawians after a year of complementary feeding with lipid-based nutrient supplements or corn-soy flour. Maternal \& Child Nutrition 2012, 8(2):239-248.

45. O'Connor LM, Lentjes MA, Luben RN, Khaw K-T, Wareham NJ, Forouhi NG: Dietary dairy product intake and incident type 2 diabetes: a prospective study using dietary data from a 7-day food diary. Diabetologia 2014, 57(5):909-917.

46. Sazawal S, Habib AA, Dhingra U, Dutta A, Dhingra P, Sarkar A, Deb S, Alam J, Husna A, Black $\mathrm{RE}$ : Impact of micronutrient fortification of yoghurt on micronutrient status markers and growth-a randomized double blind controlled trial among school children in Bangladesh. BMC Public Health 2013, 13(1):514.

47. Buyuktuncer Z, Fisunoğlu M, Guven GS, Unal S, Besler HT: The cholesterol lowering efficacy of plant stanol ester yoghurt in a Turkish population: a double-blind, placebo-controlled trial. Lipids in health and disease 2013, 12(1):91.

48. Goldbohm RA, Chorus AM, Galindo Garre F, Schouten LJ, van den Brandt PA: Dairy consumption and 10-y total and cardiovascular mortality: a prospective cohort study in the Netherlands-. The American journal of clinical nutrition 2011, 93(3):615-627.

49. Ballesta $S$, Velasco $C$, Borobio M, Argueelles F, Perea EJ: Fresh versus pasteurized yogurt: comparative study of the effects on microbiological and immunological parameters, and 
gastrointestinal comfort. Enfermedades infecciosas y microbiologia clinica 2008, 26(9):552557.

50. Pashapour N, Lou S: Evaluation of yogurt effect on acute diarrhea on 6-24 months old hospitalized infants. Turkish Journal of Pediatrics 2006, 48(2):115.

51. Olivares M, Díaz-Ropero MP, Gómez N, Sierra S, Lara-Villoslada F, Martín R, Rodríguez JM, Xaus J: Dietary deprivation of fermented foods causes a fall in innate immune response. Lactic acid bacteria can counteract the immunological effect of this deprivation. Journal of dairy research 2006, 73(4):492-498.

52. Heaney RP, Rafferty K, Dowell MS: Effect of yogurt on a urinary marker of bone resorption in postmenopausal women. Journal of the American Dietetic Association 2002, 102(11):1672-1674.

53. Derrien M, van Hylckama Vlieg JE: Fate, activity, and impact of ingested bacteria within the human gut microbiota. Trends in microbiology 2015.

54. Franz CM, Huch M, Mathara JM, Abriouel H, Benomar N, Reid G, Galvez A, Holzapfel WH: African fermented foods and probiotics. Int J Food Microbiol 2014, 190:84-96.

55. Thursby E, Juge N: Introduction to the human gut microbiota. Biochemical Journal 2017, 474(11):1823-1836.

56. Rezac S, Kok CR, Heermann M, Hutkins R: Fermented Foods as a Dietary Source of Live Organisms. Frontiers in Microbiology 2018, 9(1785).

57. Darmon N, Drewnowski A: Contribution of food prices and diet cost to socioeconomic disparities in diet quality and health: a systematic review and analysis. Nutrition Reviews 2015, 73(10):643-660.

58. Darmon N, Drewnowski A: Does social class predict diet quality? The American journal of clinical nutrition 2008, 87(5):1107-1117.

59. Maillot $M$, Darmon N, Vieux F, Drewnowski A: Low energy density and high nutritional quality are each associated with higher diet costs in French adults. The American journal of clinical nutrition 2007, 86(3):690-696.

60. Howe JA, Valentine AR, Hull AK, Tanumihardjo SA: $13 \mathrm{C}$ natural abundance in serum retinol acts as a biomarker for increases in dietary provitamin A. Exp Biol Med 2009, 234(2):140147.

61. Hotz C, Gibson RS: Assessment of Home-Based Processing Methods To Reduce the Phytate Content and Phytate/Zinc Molar Ratio of White Maize (Zea mays). Journal of Agricultural and Food Chemistry 2001, 49(2):692-698.

62. Oikeh SO, Menkir A, Maziya-Dixon B, Welch R, Glahn RP: Assessment of Concentrations of Iron and Zinc and Bioavailable Iron in Grains of Early-Maturing Tropical Maize Varieties. Journal of Agricultural and Food Chemistry 2003, 51(12):3688-3694.

63. Haug A, Christophersen O, Kinabo J, Kaunda W, Eik L: Use of dried Kapenta (Limnothrissa miodon and Stolothrissa tanganicae) and other products based on whole fish for complementing maize-based diets. African Journal of Food, Agriculture, Nutrition and Development 2010, 10(5).

64. Harris J, Haddad L, Seco Grütz S: Turning rapid growth into meaningful growth: Sustaining the commitment to nutrition in Zambia; 2014. 


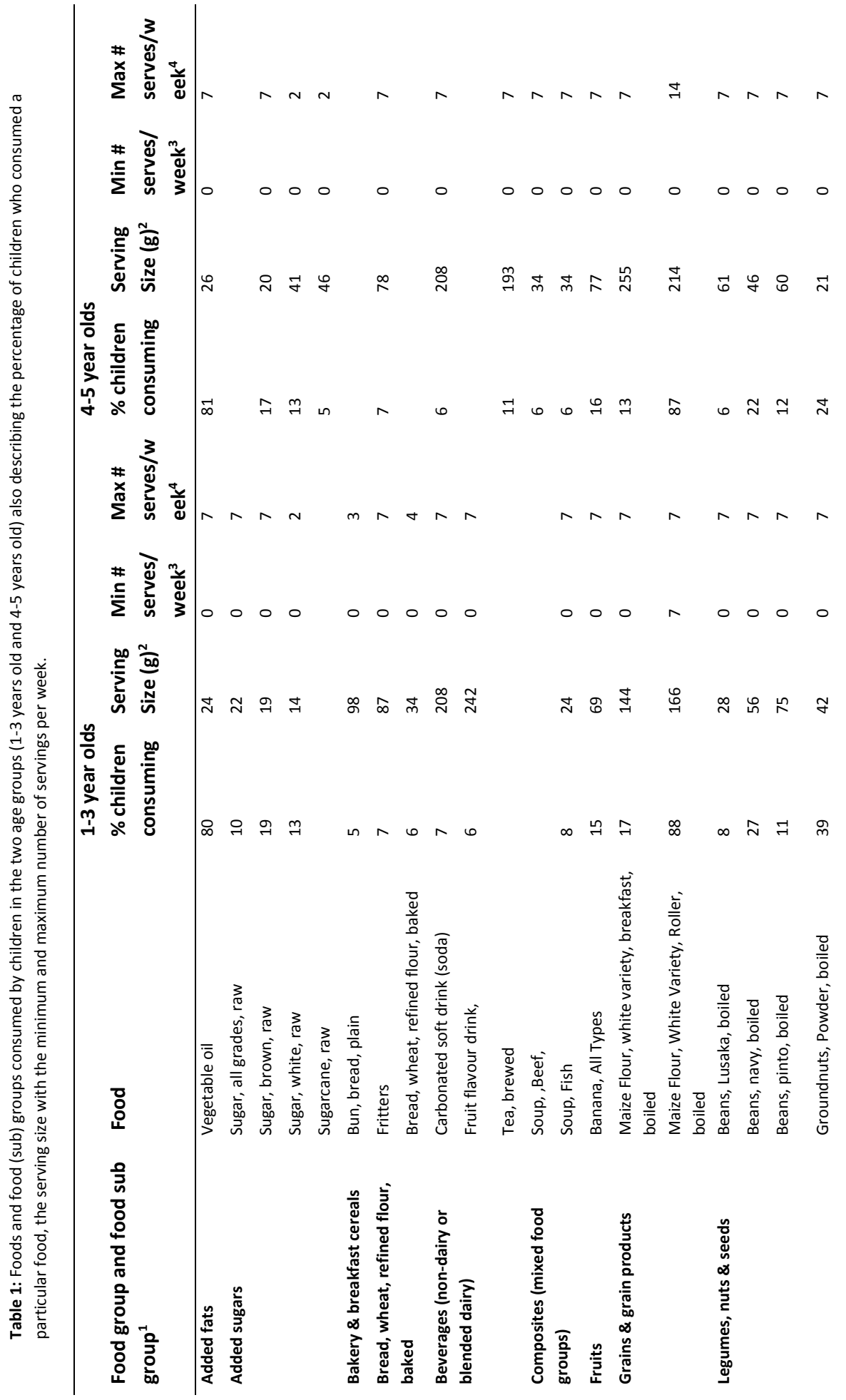




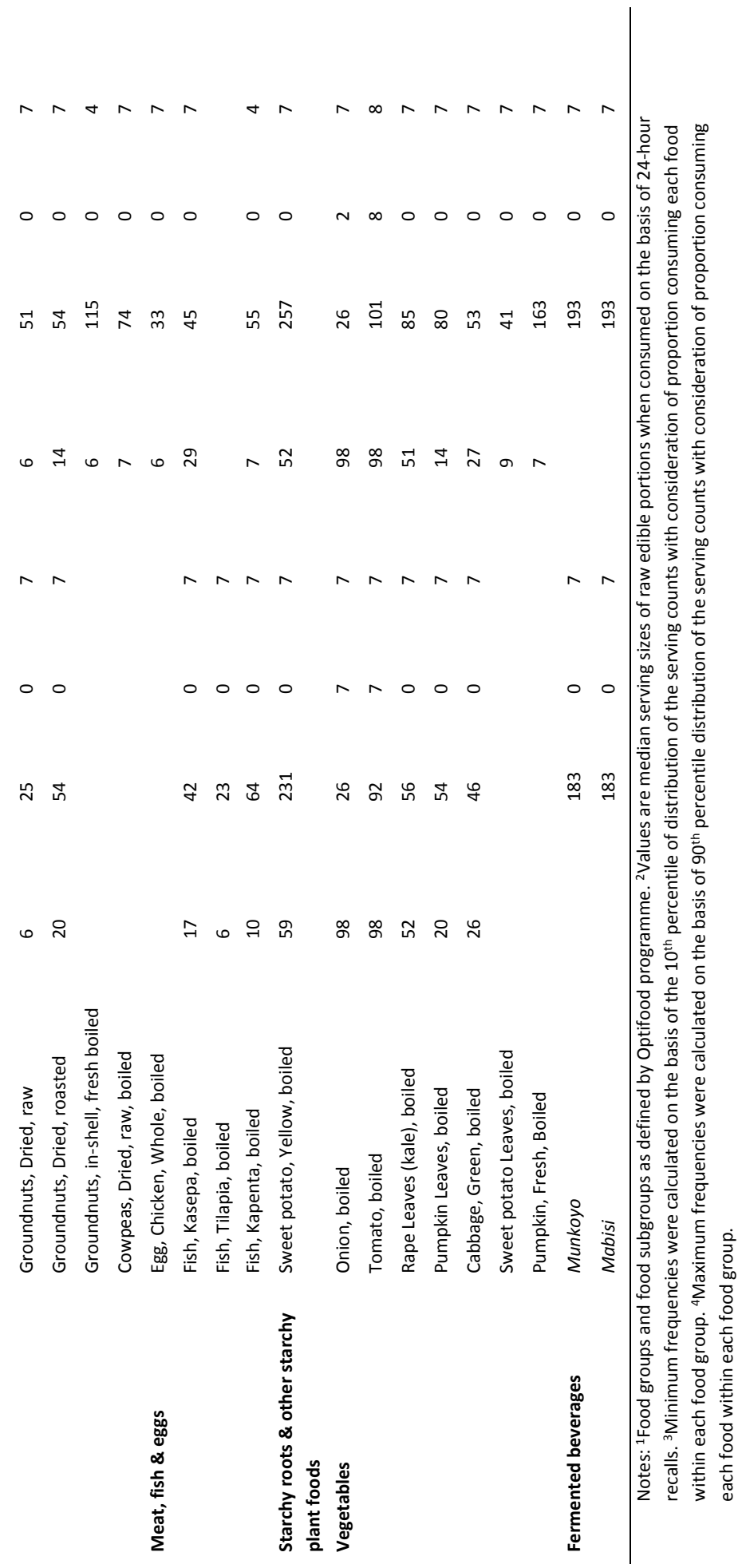




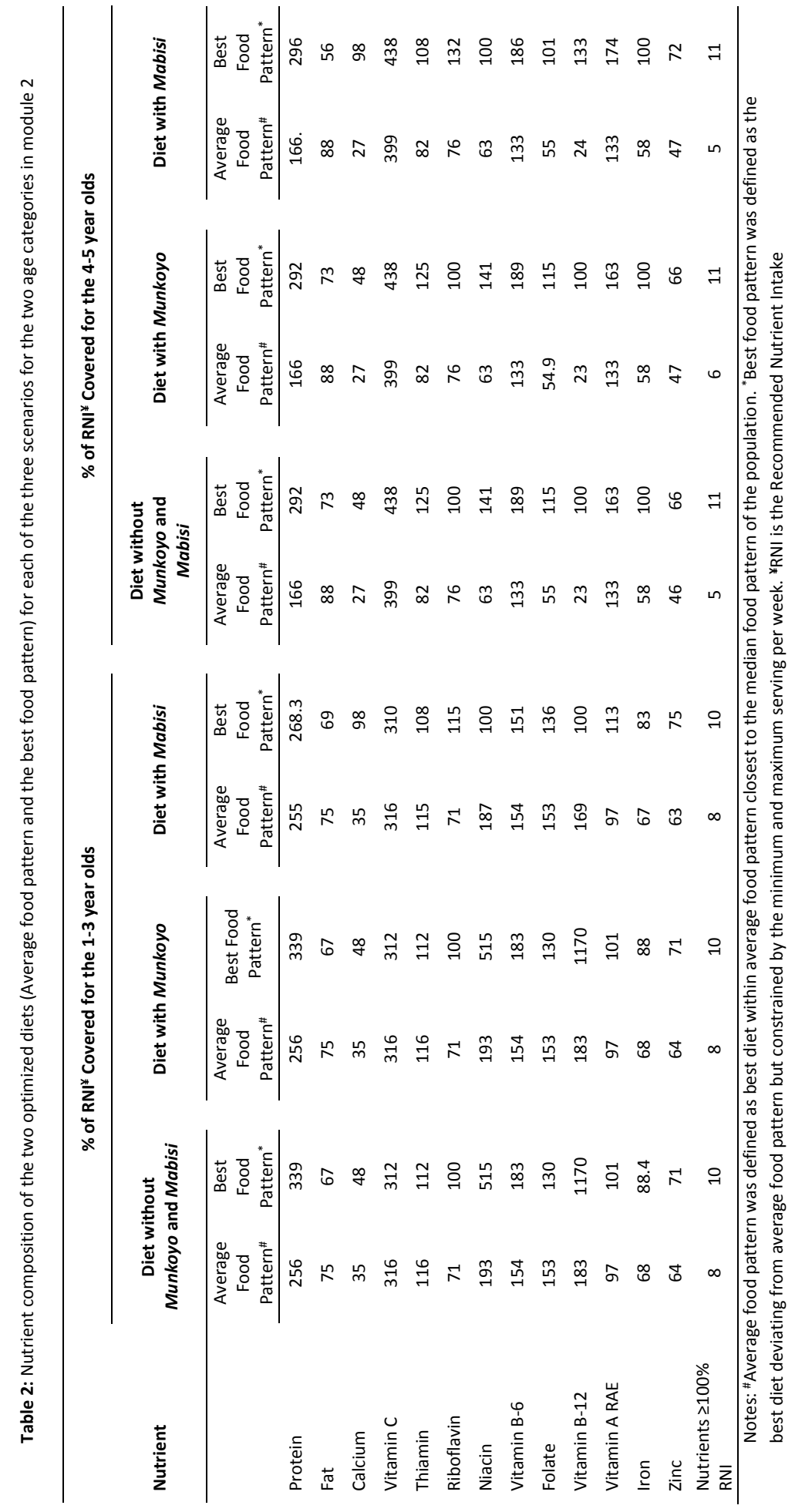




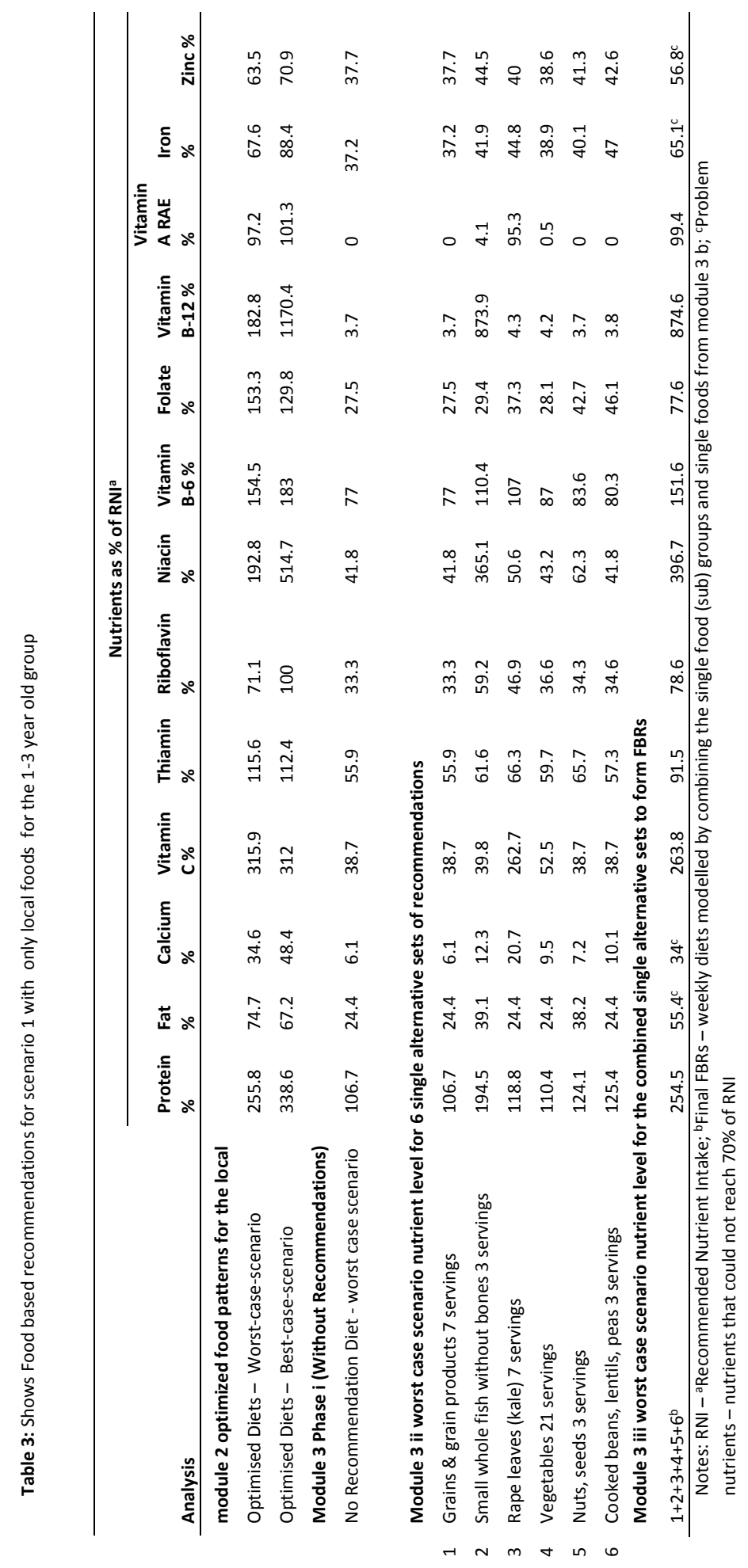




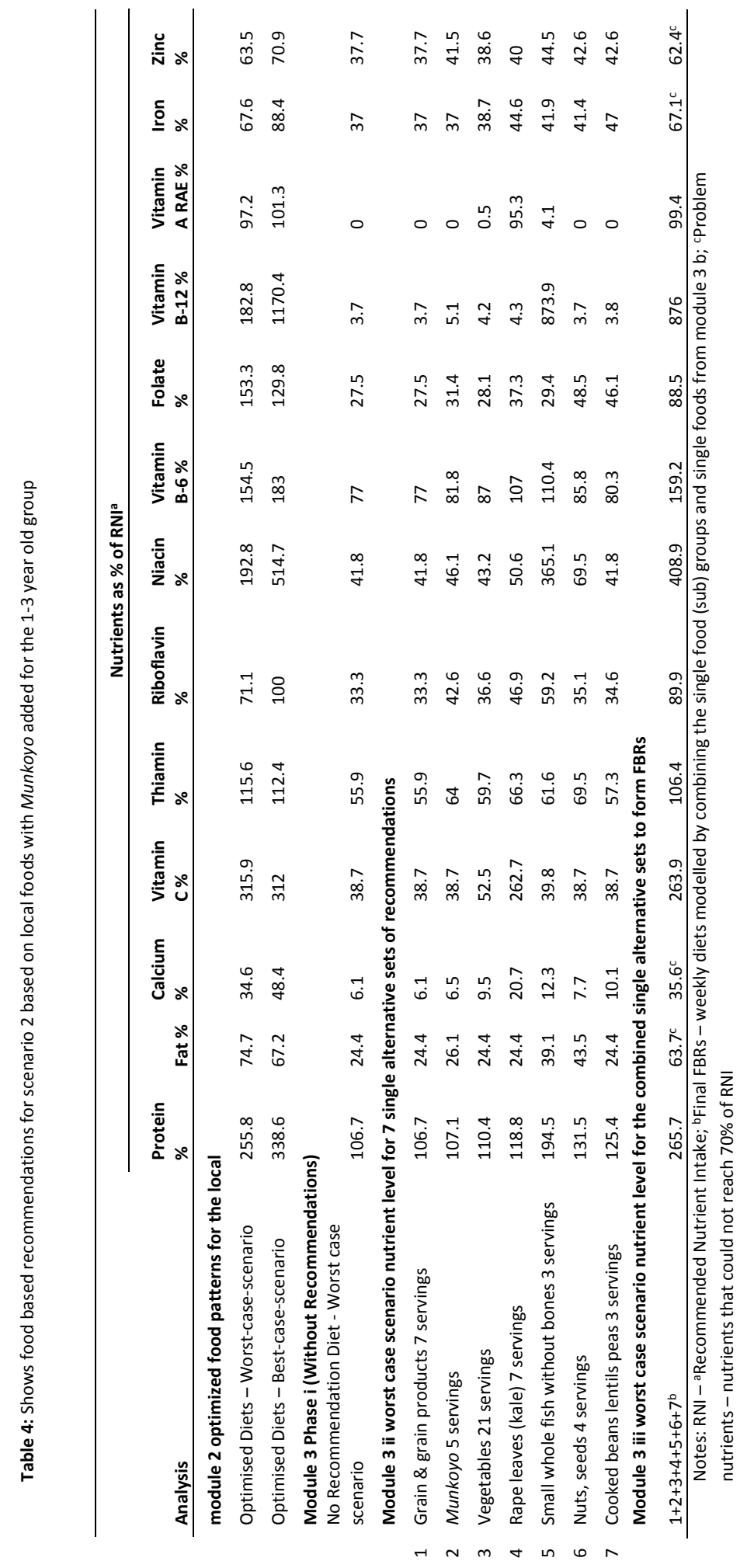




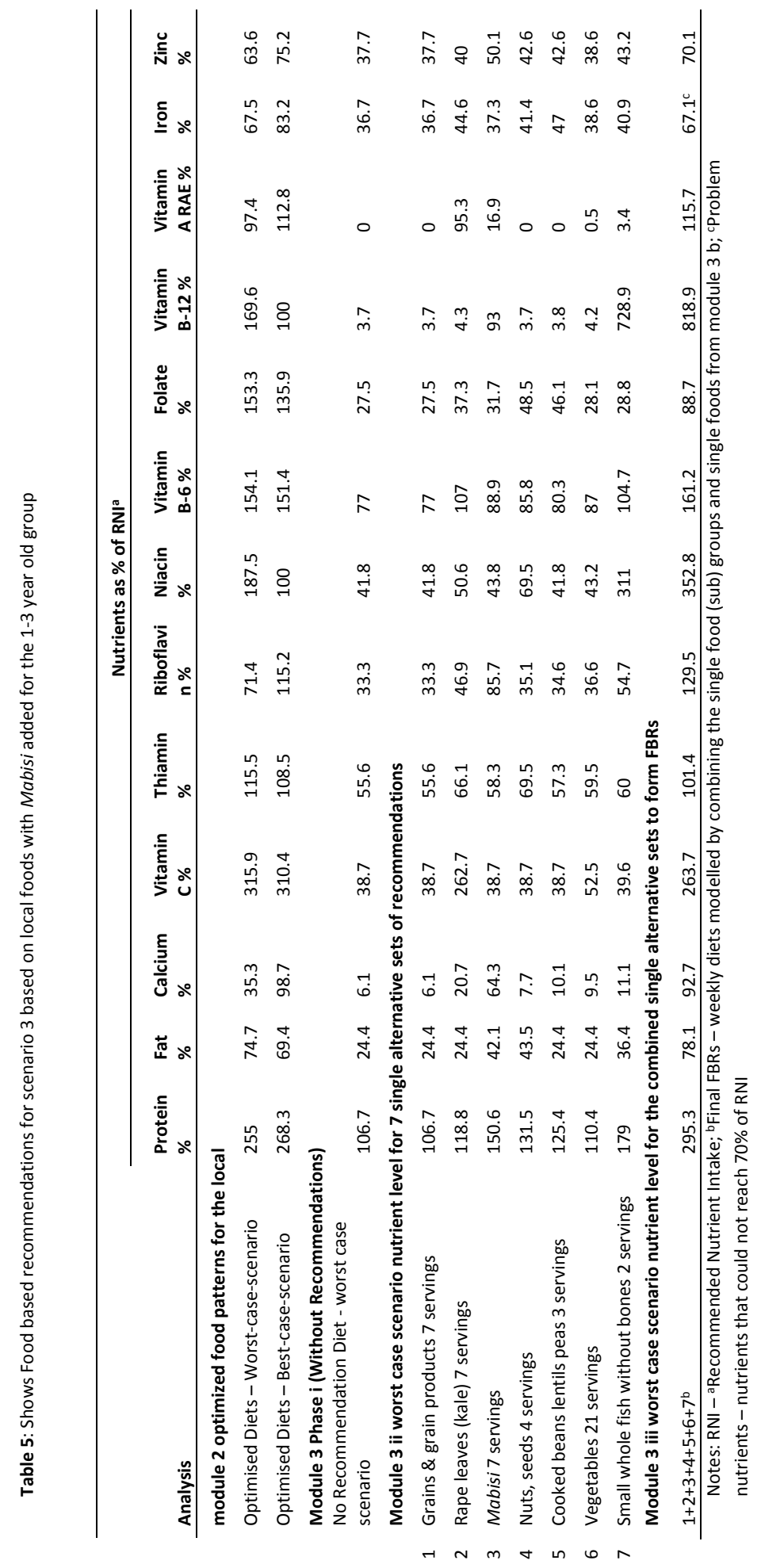




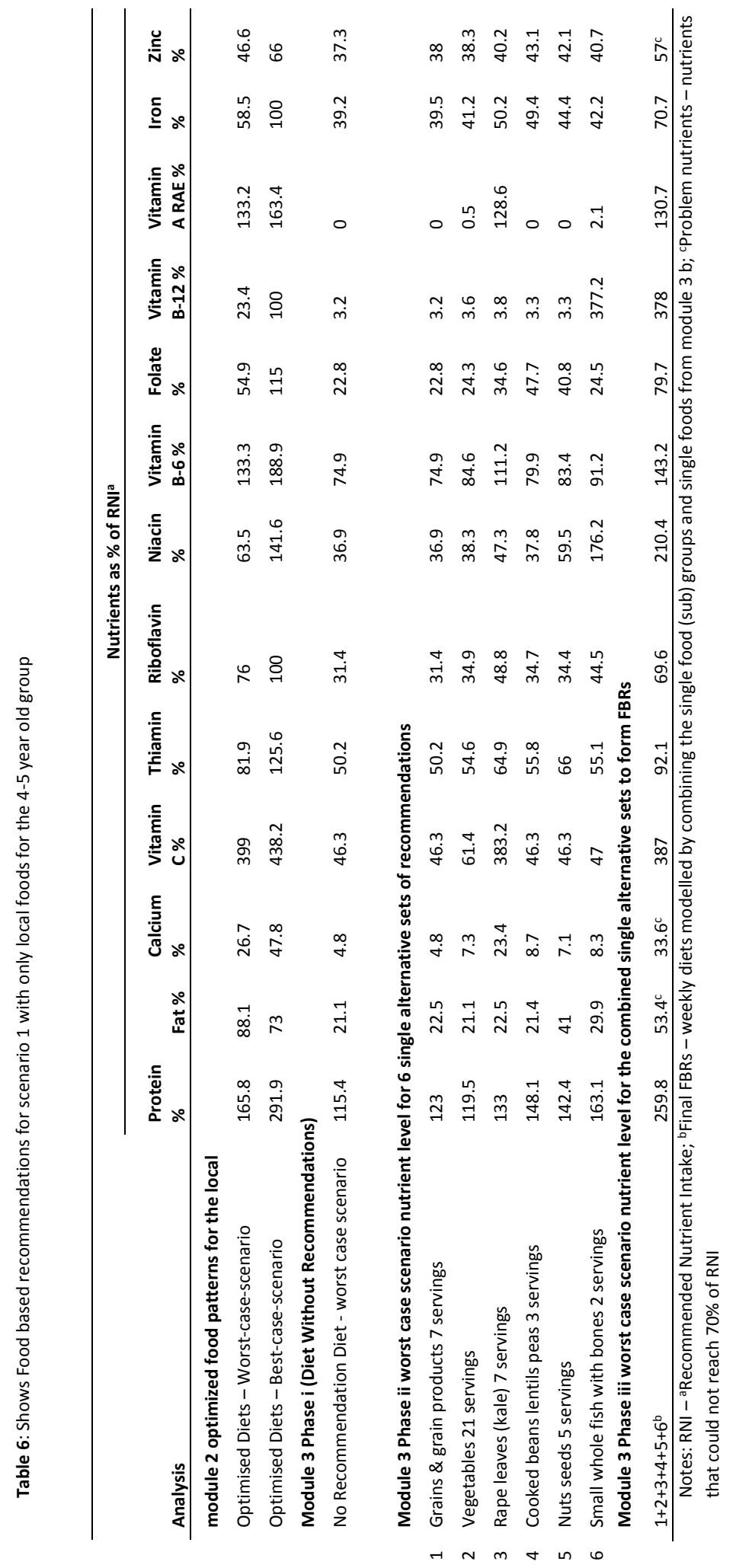




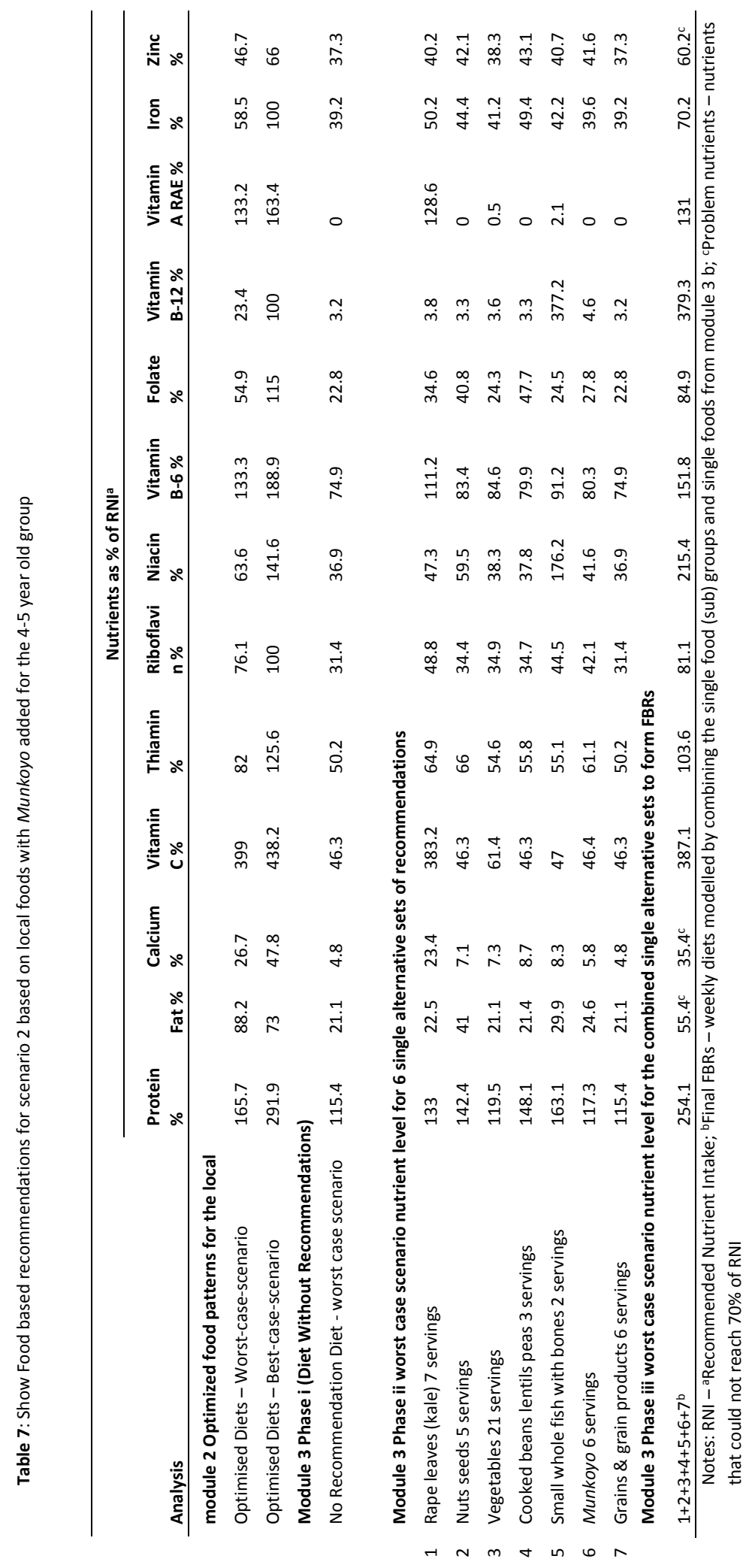




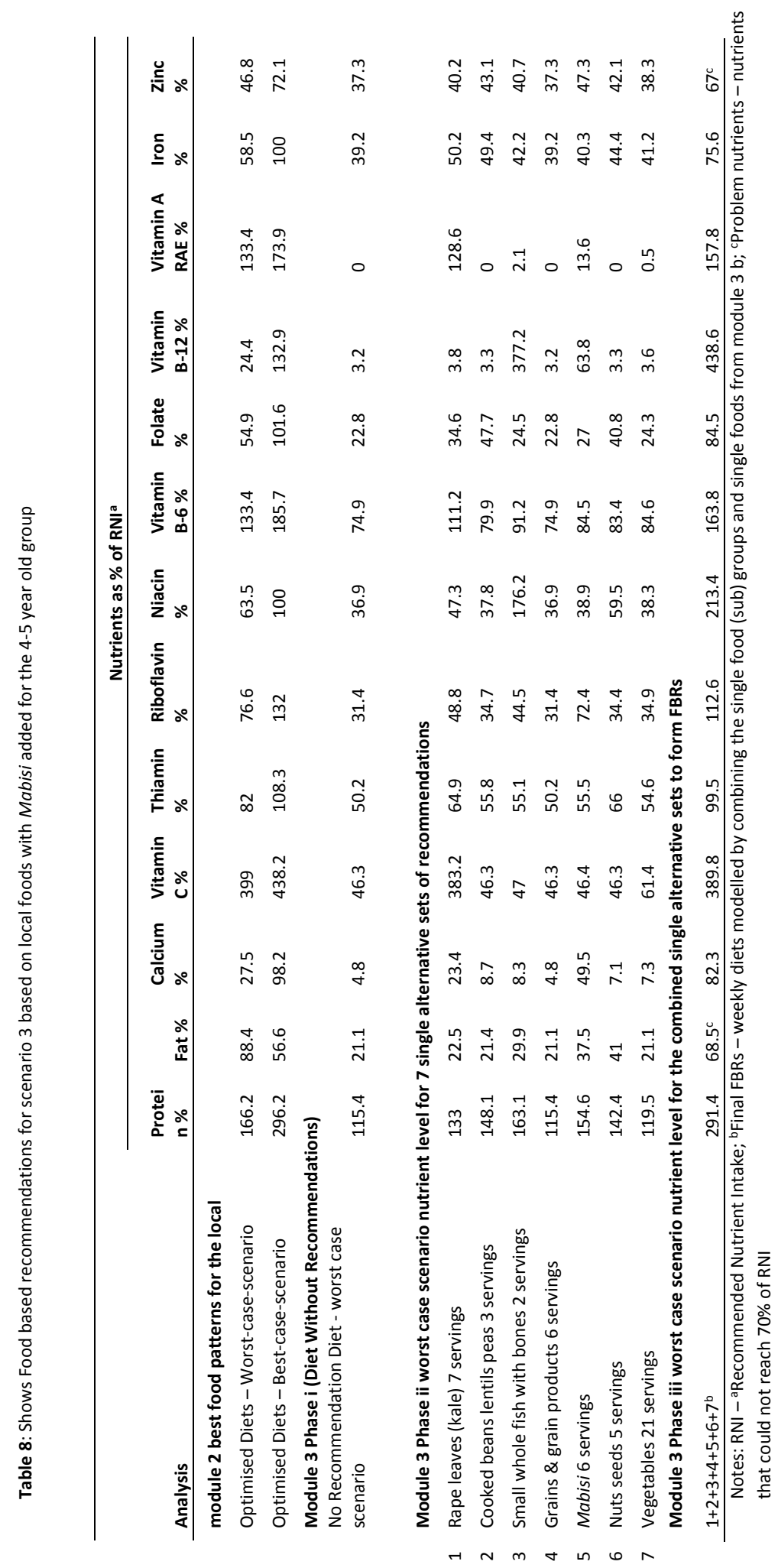


Chapter 3 
Nutritional composition and microbial communities of two non-alcoholic traditional fermented beverages from Zambia: A study of Mabisi and Munkoyo

Justin Chileshe ${ }^{1,2}$, Joost van den Heuvel ${ }^{1}$, Ray Handema ${ }^{2}$, Elise F. Talsma ${ }^{4}$, Bas J Zwaan ${ }^{1}$ and Sijmen Schoustra ${ }^{1,3}$

${ }^{1}$ Laboratory of Genetics and Division of Human Nutrition and Health, Wageningen University and Research, the Netherlands

${ }^{2}$ Tropical Diseases Research Centre, Ndola, Zambia

${ }^{3}$ University of Zambia, Department of Food Science and Nutrition, School of Agricultural Sciences, Zambia

${ }^{4}$ Division of Human Nutrition and Health, Wageningen University, the Netherlands 


\section{Abstract}

Traditional fermented foods are common in many countries, including Zambia. While the general benefits of these fermented foods, including based on their nutritional value, are widely recognised, the nutritional composition of most traditional fermented foods is unknown. Further, fermentation is known to add nutritional value (mainly B vitamins) to raw materials. In the case of traditional fermentation, the composition of microbial communities responsible for fermentation varies from producer to producer and this may also be true for the nutritional composition. Here, we characterized the nutrient profile and microbial community composition of two traditional fermented foods, milk-based Mabisi and cerealbased Munkoyo. We found that the two products are different with respect to the nutritional parameters and the microbial composition. Mabisi was found to have higher nutritional values for crude protein, fat, and carbohydrates than Munkoyo. The microbial community composition was also different for the two products, while for both dominated by lactic acid bacteria. Our analyses showed that variation in nutritional composition, defined as how much consumption would contribute to estimated average requirement (EAR), might be explained by variation in microbial community composition. Consumption of Mabisi appeared to contribute more to the estimated average requirement (\% of EAR) and its inclusion in food based recommendations is warranted. Our results show the potential of Mabisi and Munkoyo to add value to current diets and suggests that variations in microbial composition between specific product samples can result in variations in nutritional composition.

\section{Introduction}

In many countries locally processed traditional foods exist that play an important role in the diets of people. Yet, for many of these products the methods of preparation are not uniform and documented, their functional properties such as product composition, organoleptic characteristics and shelf life are unknown and the way these affect their nutritional composition has not been assessed. As a result, these local traditional foods are often not included in food-based dietary guidelines nor are there estimations of how they can contribute to local food and nutrition security.

Especially of interest are fermented foods and beverages that are produced using traditional fermentation processes. Not only are these foods locally available and part of tradition, fermentation adds value to the raw materials used, resulting in a product with prolonged shelf-life and stability and increased sensory, and monetary value $[1,2]$. The activity of microbes further is known to add nutritional value to raw materials [3], for instance by the production of B-vitamins and the removal of anti-nutritional factors such as phytate and by doing so increasing the bioavailability of various micronutrients $[4,5]$. The final nutritional and sensory properties of fermented products thus depend on the diverse microbial 
community of traditional fermented foods. In turn, the composition of the microbial community to a large extent depends on the raw ingredients of each geographical region and traditional processing procedures $[6,7]$. Apart from increased nutritional content compared to raw materials, fermented foods possess beneficial effects on human health through the modification of gut microbiota leading to better immune response, and lowering a person's risk of hypertension, diabetes, and high cholesterol [8], the prevention and treatment of inflammatory bowel disease (IBD) [9], and anti-carcinogenic and hypo-cholesterolemic effects [10]. Moreover, milk lactose is converted into lactic acid during fermentation and this has been linked to health benefits by reducing abdominal pain and diarrhoea in people with lactose intolerance [11-13].

In Zambia various traditional non-alcoholic fermented foods exist that are consumed by all age groups, of these Mabisi and Munkoyo are commonly found in rural and for Munkoyo also in some urban areas. Mabisi is produced by fermenting raw milk and Munkoyo is produced by fermenting maize porridge [14, 15]. Although consumption of both products is frequent and the product is an important part of the diet $[15,16]$, surprisingly, the nutritional composition has not been characterized. Mabisi is made by placing raw milk in a fermentation vessel and fermenting at ambient temperatures for 48 hours resulting in a mildly sour tasting product. Previous work showed that variations in processing exist, which could lead to variations in product functionality in terms of microbial composition and sensory properties $[14,15,17]$. Processing most notable differs in the repeated additions (or not) of fresh milk, the level of shaking during the fermentation, and the levels of back-slopping (transfer of material from a previous batch to a new batch, [14]).

Munkoyo is made from maize flour that is mixed with water and boiled for several hours. After cooling, Rhynchosia roots are added to provide enzymes to degrade complex sugars and to provide a microbial inoculum for fermentation $[17,18]$. Fermentation can be done in a variety of vessels at ambient temperatures and takes around 48 hours. Processing variations include the time allowed for cooking the maize porridge, the types of roots added, the fermentation vessel used, and the level of back-slopping [17]. For both products, the microbial communities responsible for fermentation are dominated by four to ten species of lactic acid and acetic acid bacteria $[14,15,17]$. The exact composition varies between samples, and variation in processing gives rise to further differentiation in microbial composition [14, 15, 17]. Since microbes affect the nutrient composition increasing the nutritional value, variations in microbial composition may lead to variation in nutritional value of the final products.

In the present study we analysed product samples of Mabisi and Munkoyo that we collected from local producers in Zambia. We documented nutritional composition of Mabisi and Munkoyo and its variation between products from different producers, in addition to profiling the microbial communities that are present at the end of fermentation. We expect to find variations in both nutritional composition and 
microbial community profiles between the different products and between samples taken of the same product. Finally, we assessed whether we could correlate variations in microbial communities to variations in nutritional content. Our study thus provides unique data on nutritional composition of two traditional fermented foods that is essential for planning of nutritional programmes in Zambia. Moreover, it provides a general outlook on the importance of understanding how microbial activity adds to the nutritional value of fermented products.

\section{Methods:}

\section{Study design}

Our study was a cross sectional study focusing on their nutritional composition and microbial community composition of Mabisi and Munkoyo. Samples were collected from the Mkushi area in Zambia (location coordinates $13.1339^{\circ} \mathrm{S}, 27.8493^{\circ} \mathrm{E}$ ). This site was selected because of the tradition of making Mabisi and Munkoyo that has been maintained by the collection of people who have migrated from other parts of Zambia to live among the locals (Swaka people). It was also logistically easier as production of both products is practiced in this area.

Samples were purchased from producers either at their homes or at the market when they were selling their products. We planned to collect 15 Mabisi samples and 15 Munkoyo samples, each from different producers, but collected 12 Mabisi and 13 Munkoyo samples. Producers were selected on the basis of location and their presence at the market in Mkushi. We asked the processors what their age (in years) and highest level of education was. Level of education was categorised as no education, attaining primary school, secondary school and tertiary education. We recorded the type of fermentation vessel that the processors had used as this may have an impact on microbial composition [19]. Samples were collected in two duplicate sterile $50 \mathrm{ml}$ centrifuge tubes with screw caps and were immediately placed in a cool box with ice packs after which they were stored in the freezer, one tube at $-20^{\circ} \mathrm{C}$ (food nutritional analysis) and the other tube at $-80^{\circ} \mathrm{C}$ (for microbial analysis). Samples were analysed at the Tropical Diseases Research Centre in Zambia for B vitamins and mineral (calcium, iron and zinc) concentration and at the University of Zambia, School of Agricultural Sciences, department of food sciences and nutrition for proximate content (protein, fibre, water content, fat, energy and carbohydrates). Samples were transported to Wageningen University in the Netherlands for whole bacterial genomic DNA extraction and $16 \mathrm{~S}$ rRNA gene sequencing.

This study was approved by the Tropical Diseases Research Centre (TDRC) Ethics review committee (Ndola, Zambia) (Ethics number STC/2015/13). 


\section{Measurement of nutritional content}

We assessed the levels of main components of the products (proximate analysis including carbohydrates, fats, protein, fibre and ash), B vitamins (B1, B2, B3, B6 and B12), and minerals (calcium, iron and zinc) using customarily used methods of analysis. We chose to measure levels of selected minerals and $B$ vitamins since these are the most relevant considering the raw materials used in the production of Mabisi (milk) and Munkoyo (maize). We calculated what the contribution of one woman serving of the product amounting to $183 \mathrm{~g}$ [16] would contribute towards the estimated average requirement (\% of EAR) for each of the nutritional components.

Proximate analysis: Different proximate parameters in Munkoyo and Mabisi were determined using the methods of the Association of Official Analytical Chemists (AOAC) [20]. Briefly, crude fat was measured in samples using Soxhlet apparatus with hexane as the solvent in the AOAC procedure. Protein was determined using nitrogen content by the micro-Kjeldahl method where nitrogen value obtained for each sample was converted to crude protein by multiplying with the 6.25 factor. Moisture and dry matter content were determined by weighing $2 \mathrm{~g}$ of sample onto a crucible, heating to dryness in an oven at $110^{\circ} \mathrm{C}$ for 2 hours and calculating the weight difference. Crude fibre was determined in each sample, after removal of fat, using successive digestion with $1.25 \%$ sulphuric acid and $1.25 \%$ sodium hydroxide solutions. Carbohydrate content was determined by difference calculation method as follows: \% Total Carbohydrate $=[100-\%($ Protein + Fibre + Ash + Fat + Moisture $)]$. All the proximate parameters were reported in AOAC, 2000 standard format as percentage.

Atomic Absorption Spectrophotometry (AAS): The contents of calcium, iron, and zinc were determined by dry-ashing of samples at $450^{\circ} \mathrm{C}$ in a muffle furnace following procedures described earlier [21]. After dissolving of the resulting ash in hydrochloric acid ( $\mathrm{HCL}$ ) the metal element contents in the solutions were determined by flame atomic absorption spectrophotometry (AAS) (AAnalyst-400, Perkin-Elmer Corp., Norwalk, CT, USA). Standards were prepared from stock standard solutions (1000 ppm) of zinc, iron, and calcium to make a calibration curve for each element. Analyses were performed in duplicate.

Quality control $(Q C)$ : To monitor performance and reproducibility of the analytical procedures used, we included quality control samples for each batch of samples on a daily basis. For calcium and iron we used Cobas $^{\mathrm{TM}}$ control samples while for zinc we used inhouse QC samples with values previously determined using the Seronorm ${ }^{\mathrm{TM}}$ Trace Elements Serum Level 1 and 2. The means, standard deviations and coefficient of variation (CV\%) for duplicate samples were calculated to ensure that the values were within the acceptable limits (10\%).

Analysis of B-Vitamins (B1, B2, B3, B6, and B12) by High Pressure Liquid Chromatography (HPLC): A previous method based of HPLC UV analysis was used with a few modifications [22]. After the 
homogenization of the sample by mixing, $2 \mathrm{~g}$ was weighed in a $100 \mathrm{ml}$ volumetric flask, followed by adding $40 \mathrm{ml}$ of water and $4 \mathrm{ml}$ of $2 \mathrm{M} \mathrm{NaOH}$. The suspension was then vigorously shaken, then $50 \mathrm{ml}$ of $1 \mathrm{M}$ phosphate buffer $(\mathrm{pH} 5.5)$ was added in order to lower the $\mathrm{pH}$ of the final solution to about $\mathrm{pH}$ 7. The suspension was made up to the mark with water and sonicated for 10 minutes in an ultrasonic bath. Dilutions of 20 -fold with water were used for the quantification of the vitamins. The solution was filtered through a $0.22 \mu \mathrm{m}$ Millipore syringe before analysis. Analyses were performed in duplicate.

Standard preparation: The multi-vitamins stock solution was prepared by weighing in a $100 \mathrm{ml}$ volumetric flask $5 \mathrm{mg}$ of vitamin B12; $12.5 \mathrm{mg}$ of vitamin B2; $25 \mathrm{mg}$ each of vitamins B1, B6, and B3. Forty millilitres $(40 \mathrm{ml})$ of water was then added and the solution was shaken vigorously before adding $4 \mathrm{ml}$ of $2 \mathrm{M} \mathrm{NaOH}$. After complete dissolution of the vitamins, $50 \mathrm{ml}$ of $1 \mathrm{M}$ phosphate buffer $(\mathrm{pH} 5.5)$ was added and the solution made up to the mark with water. Stock standard solutions were prepared daily. Different concentrations of the standards were injected into the HPLC to obtain the peak areas. Peak areas were plotted against concentration for each vitamin to make specific calibration curves. The Shimadzu LC2010CHT HPLC system was used with conditions that have been obtained before in other studies [23]. A volume of $20 \mu \mathrm{l}$ for each sample was injected into the HPLC equipped with C18 reversed phase Column $(250 \times 4.6 \mathrm{~mm}, 4 \mu \mathrm{m}), 0.05 \mathrm{M}$ ammonium acetate (solvent A)-methanol (solvent B) 92.5:7.2 as mobile phase at $1 \mathrm{ml} /$ minute flow rate. A diode array detector was used to scan from 200 to $500 \mathrm{~nm}$ and LCsolutions software (Shimadzu, Japan) was used to integrate the peak areas for each vitamin. After the run, the peak area of each unknown sample was obtained, and concentrations were calculated using the calibration curves.

Quality control: To monitor performance and reproducibility of the analytical procedures for the analysis of the B vitamins, we included quality control samples spiked with known amounts of standards for each batch of samples on a daily basis. The means, standard deviations and coefficient of variation (CV\%) for duplicate samples were calculated to ensure that the values were within the acceptable limits $\leq 10 \%$.

\section{Characterization of microbial composition of Mabisi and Munkoyo}

We characterized microbial community composition to assess whether or not variation in nutritional composition could be attributed to variation in microbial community composition. This may be the case since the microbes found in earlier work on Mabisi and Munkoyo are generally associated with production of some of the B vitamins.

Total genomic DNA extraction: Sample DNA was extracted from Mabisi and Munkoyo samples using the method by Schoustra et al. [15]. Briefly, for Munkoyo, after eliminating large particles from $1 \mathrm{ml}$ of product samples were spun down at high speed, the pellet was retained after discarding the supernatant. Then $500 \mu \mathrm{l}$ TESL (25 mM Tris, $10 \mathrm{mM}$ EDTA, $20 \%$ sucrose, $20 \mathrm{mg} / \mathrm{ml}$ lysozyme) and $10 \mu \mathrm{l}$ mutanolysin solution 
(in water at $1 \mathrm{U} / \mu \mathrm{l}$ ) were added, followed by incubation at $37^{\circ} \mathrm{C}$ for 60 minutes with slight shaking. GES reagent ( $5 \mathrm{M}$ guanidium thiocyanate, $100 \mathrm{mM}$ EDTA, $0.5 \%$ sarkosyl) amounting to $500 \mu \mathrm{l}$ was added, cooled on ice for 5 minutes and $250 \mu \mathrm{l}$ of cold ammonium acetate solution (7.5 M) was added followed by gentle mixing. The mixture was held on ice for 10 minutes, spun down and the supernatant removed. The samples were purified by mixing with chloroform-2-pentanol mix (chloroform and 2- pentanol 24:1 ratio) by adding $1: 1$ to the supernatant and the mixture was centrifuged to obtain the supernatant. Phenol-chloroform purification was performed by adding equal volume of phenol (i.e., tris-saturated phenol-chloroform-isoamyl ethanol in a ratio of 24:25:1) to the supernatant, vortexed for a few seconds, spun for 2 minutes at $12000 \mathrm{rpm} 4^{\circ} \mathrm{C}$ and the supernatant was transferred to a fresh tube. An equal volume of chloroform was added to the supernatant, vortexed for a few seconds, spun 2 minutes at 12000 rpm and $4^{\circ} \mathrm{C}$ and transferred the supernatant to a fresh tube. An amount 2.5 volumes $100 \%$ ethanol was added, vortexed and precipitated the DNA at $-80^{\circ} \mathrm{C}$ for 3 hours. Subsequently, samples were spun for 20 minutes at $12000 \mathrm{rpm}$ and $4^{\circ} \mathrm{C}$; the supernatant was removed by aspiration. DNA was washed by adding $1 \mathrm{~mL}$ cold $70 \%$ ethanol, spun for 10 minutes at $12000 \mathrm{rpm}$ and $4^{\circ} \mathrm{C}$; the supernatant was removed by aspiration and the DNA pelleted was air-dried for 10 minutes at room temperature. The DNA was dissolved in $10 \mathrm{mM}$ Tris treated with RNAse (10 mM Tris, bring to $\mathrm{pH} 8.0$ with $\mathrm{HCl}$; 1 mM EDTA; RNAse 20 $\mu \mathrm{l} / \mathrm{ml}$ ) and stored at $-20^{\circ} \mathrm{C}$.

For the milk-based product (Mabisi) the DNA extraction protocol was performed as follows: into a $1.5 \mathrm{ml}$ microcentrifuge tube was added $1 \mathrm{ml}$ of Mabisi and centrifuged at 13,000 $\times \mathrm{g}$ for 2 minutes to pellet the cells and removed the supernatant. The cells were re-suspended in a solution containing $64 \mu \mathrm{l}$ of a $0.5 \mathrm{M}$ EDTA solution, $160 \mu \mathrm{l}$ of Nuclei Lysis Solution (Promega), $5 \mu \mathrm{l}$ RNase $(10 \mathrm{mg} / \mathrm{ml}), 120 \mu \mathrm{l}$ lysozyme (10 $\mathrm{mg} / \mathrm{ml})$ and $40 \mu \mathrm{l}$ protenase $\mathrm{E}(20 \mathrm{mg} / \mathrm{ml})$ and incubated for 60 minutes at $37^{\circ} \mathrm{C}$. Ammonium acetate $(5$ M) $400 \mu \mathrm{l}$ was added and cooled on ice for 15 minutes before being spun down at 13,000 $\times \mathrm{g}$ for 10 minutes. The supernatant containing the DNA was transferred to a fresh $1.5 \mathrm{ml}$ microcentrifuge tube and a phenol-chloroform DNA purification was performed as described for Munkoyo.

16SrRNA amplicon sequencing of DNA samples and analysis of sequence data: the Company LGC Genomics $\mathrm{GmbH}$ (Germany) conducted 16S rRNA gene analysis of bacterial communities in metagenomic DNA samples using the illumina MiSeq V3. Using an analysis pipeline [19] based on qiime software the 25 samples collected from the producers in Mkushi were analyzed. Firstly, the forward and reverse reads were joined in one fastq sequence (join_paired_ends.py, minimum overlap 10 nucleotides). Then primers were removed from both ends and reads were quality trimmed (using cutadapt, minimum length 400, minimum quality 20). Indeed, no adapters were present in the data. With uchime, chimeric reads were removed (using blast against the "gold" database). Then the sequences were given identifier names by a custom awk script (similar to split_libraries.py). Then otus were determined using 
pick_open_reference_otu.py, at 0.95 similarity and with this script OTU clusters were produced, taxonomies were assigned and an OTU table was produced. From the OTU table produced, the minimum number of sequences per sample was determined and OTU tables were made by using multiple_rarefactions.py, to 15000 sequences. Then alpha diversity and beta diversity were determined, which produced the distance matrices that were used for jackknife clustering (upgma_cluster.py), from which a consensus tree was produced (consensus_tree.py). Bacterial diversity by total effective sequence reads, Operational Taxonomic Units (OTU) numbers, Chao1, and the Faith's phylogenetic diversity (PD_Whole_Tree) were used to evaluate and compare diversity and richness of the communities among different samples, i.e. between and within sample types (Product type).

\section{Statistical analysis}

We used R statistical package version 3.5.0 and IBM SPSS statistics version 25 to analyze the data. The nutrition composition data was presented as means with standard deviation and percentage coefficient of variation. A student t-test and ANOVA as group tests were performed and principal components analysis (PCA) was carried out to determine variation in the samples. The nutritional variables were each categorized into three classes (low, medium, high) based on the percentage they can contribute to the estimated average requirement (EAR). The values that are able to contribute less than $20 \%$ of EAR were taken as low, for contribution between 20 and 50\% they were taken as medium, and for contribution of $50 \%$ and above then they were regarded as high.

For this study, we focused on the bacterial composition alone, since earlier work has shown that yeast and other eukaryotes are usually present at an abundance below 1\% [15]. The alpha diversity indices Faith's phylogenetic diversity measure (PD) and Chao1 were calculated for Mabisi and Munkoyo samples. Comparisons of diversity between Mabisi and Munkoyo samples were done using t-test of Chao1 diversity index. Then a non-parametric test, analysis of similarities (ANOSIM), was used to determine the impact of various independent variables including product type, fermentation vessel, and categories of nutritional parameters, on the dependent variable microbial composition. This is a form of hierarchical clustering to assess whether the observed differences in microbial composition between the samples could be attributed to various design variables (nutritional parameters). This analysis shows whether or not classifying the samples in distinct groups explains significant parts of the variation in microbial species composition between the samples. 


\section{Results}

We collected 12 Mabisi and 13 Munkoyo samples in Mkushi from different processors, each processor producing only one of the two products. Mabisi producers were two males and ten females, whereas all Munkoyo producers were females whereas. The average age for Mabisi producers was 29 years $(S D=2.598 ; \min =18, \max =46$ ) and for Munkoyo producers was 41.3 years $(S D=3.542 ; \min =26, \max =62$ ). Education levels of the Mabisi producers were that five completed high school and seven attained primary school education whereas for the Munkoyo producers three completed high school, seven completed primary school and three did not have any education. The fermentation vessels used by the local processors for Mabisi were small plastic bottles (83\%) and plastic buckets (17\%), whereas for Munkoyo it was mostly calabashes (62\%) and metal drums (38\%). Samples were analyzed for their nutritional and microbial composition.

\section{Nutritional analyses}

For the nutritional composition, the results of the proximate analysis are presented in Table 1, and levels of vitamins and minerals are in Table 2 . The quality control revealed that all duplicate samples were below the acceptable range of $10 \%$ CV for AAS and HPLC. Mabisi had a moisture content of $88 \%$, with a few exceptions with a moisture content of $70 \%$, while Munkoyo had a moisture content mostly between 90 and $95 \%$. The EAR as calculated by FAO/WHO [24] for women 19-50 years old used for each nutrient in this study are shown in Table 5 with iron estimated at $5 \%$ bioavailability and zinc at low bioavailability. Consumption of one serving (183 g) of Mabisi would contribute to intake of protein ( $18 \%$ of EAR), vitamin B2 (27\% of EAR), zinc ( $15 \%$ of EAR) and calcium ( $22 \%$ of EAR) and to less than $10 \%$ of the EAR for each of the other B-vitamins (tables 1 and 2). Consumption of one serving of Munkoyo would contribute to less than $10 \%$ of EAR for each of the nutrients. For each individual nutritional parameter that we measured, values are higher for Mabisi than Munkoyo except vitamin B1, vitamin B3 and vitamin B6.

The similarity between the products in their B-vitamin composition and differences in the other nutritional parameters is also reflected in the principal component analysis (PC-analysis) shown in Figure 1 and Table 3. We found that principal components 1 and 2 together explained about $77.4 \%$ of the variation between the samples. Principal component 1 (PC1) explains $65.6 \%$ of all variation with nutritional parameters high in positive loadings including calcium, ash, vitamin B2, vitamin B12, crude protein, carbohydrates, iron, energy, $\mathrm{pH}$, crude fat, vitamin $\mathrm{B} 3$, and moisture (with a negative loading) as shown in supplementary Table 6. Principle component 2 (PC2) explains $11.8 \%$ of variation with variables high in loadings including vitamin B1 and vitamin B6. The PC-analysis clearly separates Mabisi and Munkoyo samples showing differences between the two products as indicated with small blue circles in Figure 1. The nutritional parameters are all aligned around zero for $\mathrm{PC} 2$ except for vitamins $\mathrm{B} 1, \mathrm{~B} 3$, and B6, which were the reason why Munkoyo 
samples were differentiated along PC2. The vitamins B1, B3, and B6 were similar between Mabisi and Munkoyo samples but not the other nutritional parameters. The difference was confirmed using a $t$-test with results indicating that there was no statistically significant difference between Mabisi and Munkoyo for vitamin $\mathrm{B} 1$ [ $\mathrm{t}(23)=0.543, \mathrm{P}=0.592]$; for vitamin $\mathrm{B} 3[\mathrm{t}(23)=1.906, \mathrm{P}=0.069]$; and for vitamin $\mathrm{B} 6$ $[t(23)=1.865, P=0.075]$.

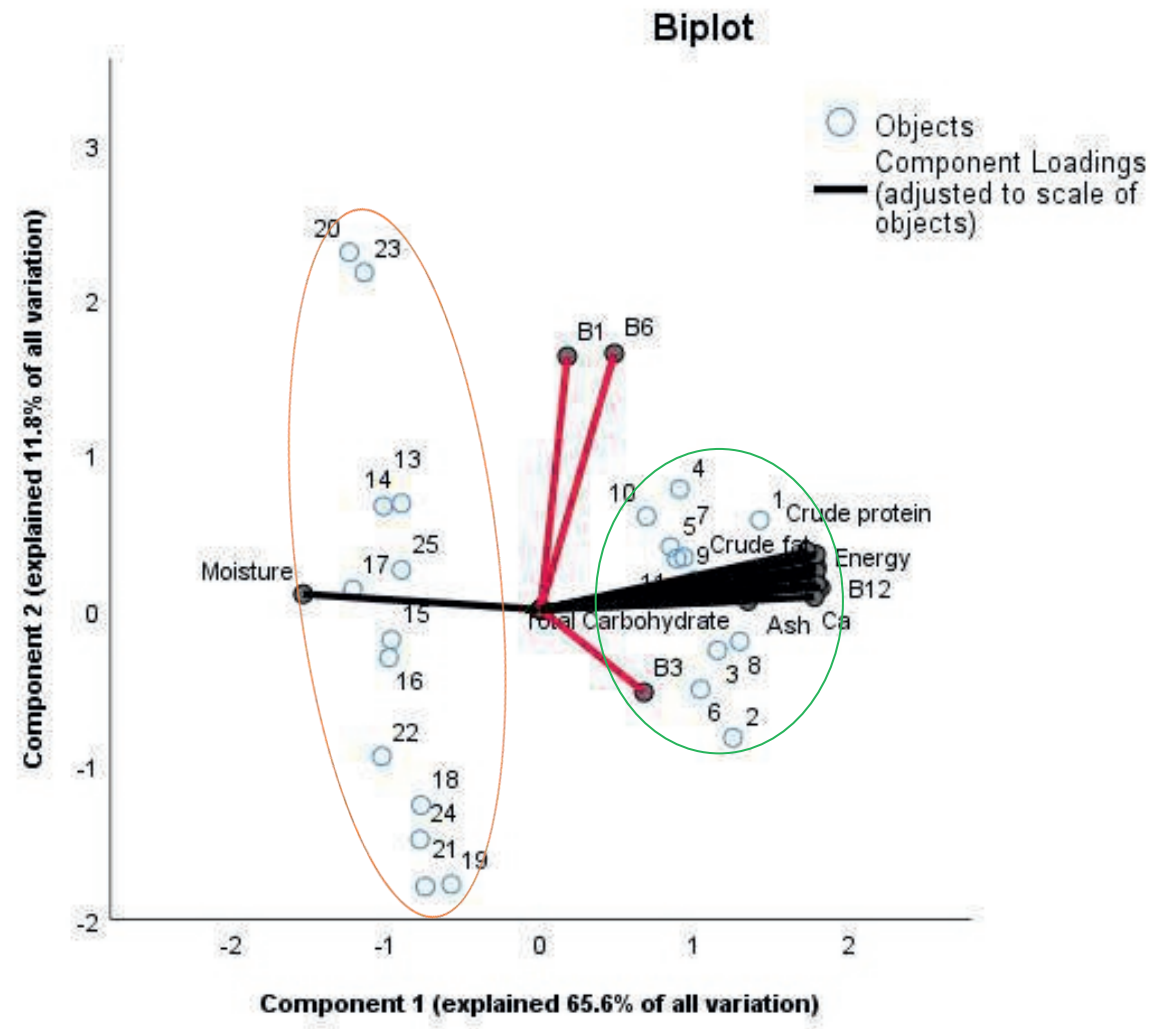

Figure 1: Principal Components Analysis (PCA) bi-plot with Optimal Scaling of Mabisi (numbers 1 to 12) and Munkoyo (numbers 13 to 25 ) samples indicated as blue circles to determine variation in nutritional parameters within and in between sample types. PC1 explained $65.6 \%$ of all variation while PC2 explained $11.8 \%$ of all variation giving a total of $77.4 \%$ variation explained by the 2 components. The Munkoyo samples are spread along PC2 and the variation is explained by vitamin B1 and B6 with higher loadings as in Table 1. Orange ellipse indicates Munkoyo samples with ID numbers 13 to 25; Green ellipse indicates Mabisi samples with ID numbers 1 to 12. Imposed on the graph are the nutritional parameters with vitamins B1, B3 and B6 (indicated by the red lines) separated away from the rest as they were not different between Mabisi and Munkoyo; also moisture clustered with Munkoyo samples as it was higher in Munkoyo than Mabisi; other nutritional parameters are clustered with Mabisi samples as they were higher in Mabisi samples than in Munkoyo samples. 


\section{Microbial analyses}

Microbial community composition for each sample of Mabisi and Munkoyo as determined by non-culturebased methods are shown in Figure 2. In total 1826 distinct bacterial types (Operational Taxonomic Units or OTUs) were found in all samples of which most were identified as either Lactobacillus, Lactococcus, Streptococcus, Enterobacter, Klebsiella, or Acetobacter.

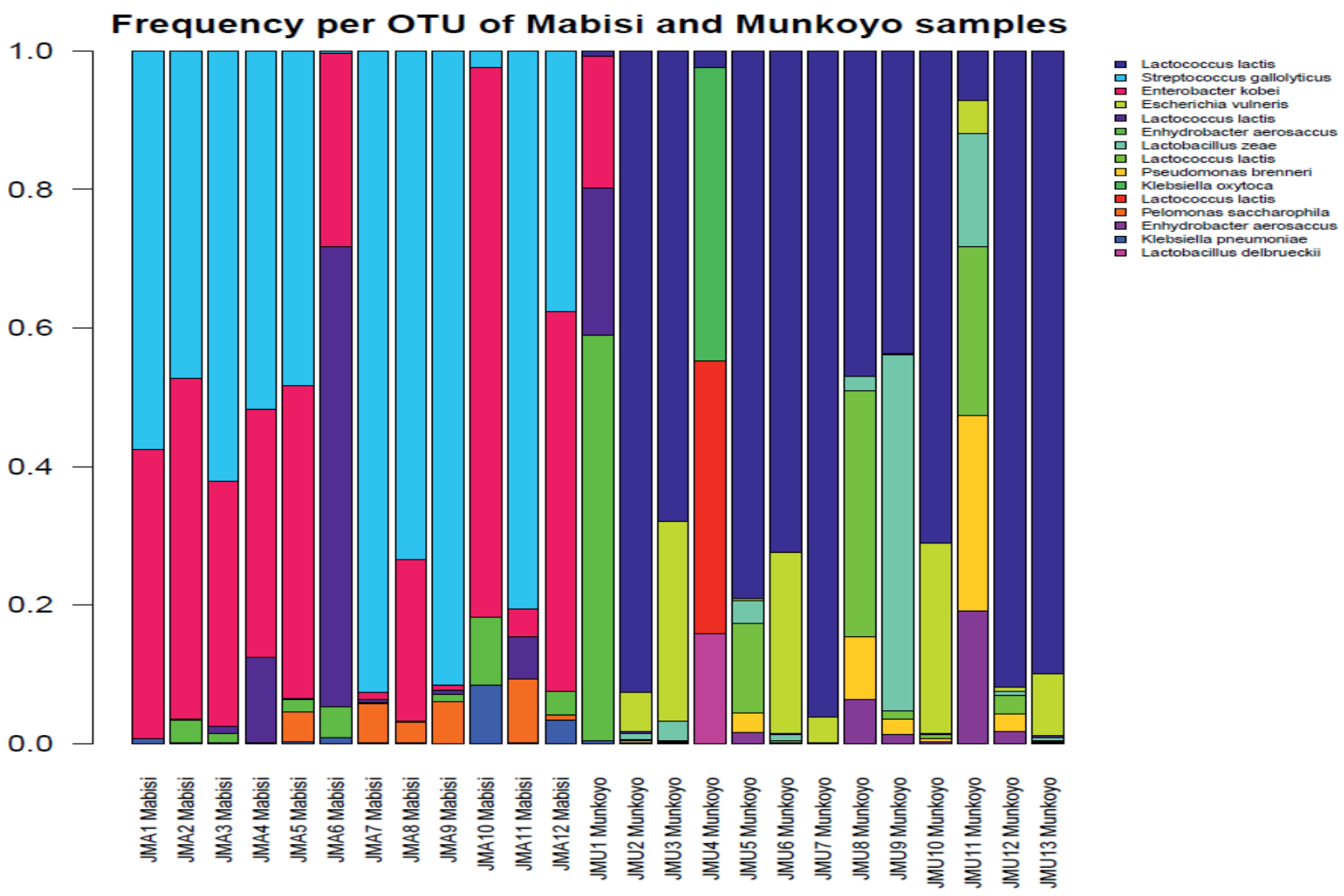

Figure 2: Relative abundance of bacterial types that constitute the bacterial communities in Mabisi and Munkoyo samples with the $y$-axis representing relative abundance and the $x$-axis representing the different samples.

We calculated two diversity indices to describe the microbial communities in the samples. The alpha diversity indices Faith's phylogenetic diversity measure (PD) and Chao1 were calculated for Mabisi and Munkoyo samples and results are shown in Table 3. Based on Chao1 alpha diversity index, Mabisi samples had higher species richness than Munkoyo samples as shown in Figure 3 and the statistically significant difference was confirmed by a t-test $[t(23)=-7.18, P<0.001]$. Based on Faith's PD, there was no difference in microbial diversity between Mabisi and Munkoyo samples. 
Table 3: Results of Mabisi and Munkoyo alpha diversity measures (at highest sampling rarefaction of 15000 sequences per samples) for each sample based on Chao1 and Faith's Phylogenetic Diversity (PD) .

\begin{tabular}{cccccc}
\hline & \multicolumn{2}{c}{ Munkoyo } & & \multicolumn{2}{c}{ Mabisi } \\
Sample ID & Chao1 & Faith's Phylogenetic Diversity & Sample ID & Chao1 & Faith's Phylogenetic Diversity \\
\hline MU1 & 148.4 & 2.4 & MA1 & 309.2 & 2.9 \\
MU2 & 142.4 & 2.2 & MA2 & 470.4 & 2.8 \\
MU3 & 103.8 & 2.0 & MA3 & 206.2 & 2.3 \\
MU4 & 155.5 & 2.0 & MA4 & 339.1 & 3.4 \\
MU5 & 98.9 & 2.0 & MA5 & 471.1 & 3.2 \\
MU6 & 197.1 & 3.4 & MA6 & 262.4 & 2.4 \\
MU7 & 85.8 & 2.1 & MA7 & 357.0 & 2.6 \\
MU8 & 140.2 & 2.4 & MA8 & 185.9 & 1.7 \\
MU9 & 88.0 & 3.9 & MA9 & 341.3 & 2.6 \\
MU10 & 223.1 & 2.9 & MA10 & 289.8 & 2.5 \\
MU11 & 95.5 & 2.3 & MA11 & 283.5 & 2.3 \\
MU12 & 117.1 & 2.2 & MA12 & 311.5 & 2.6 \\
MU13 & 208.3 & 2.7 & & & \\
\hline
\end{tabular}
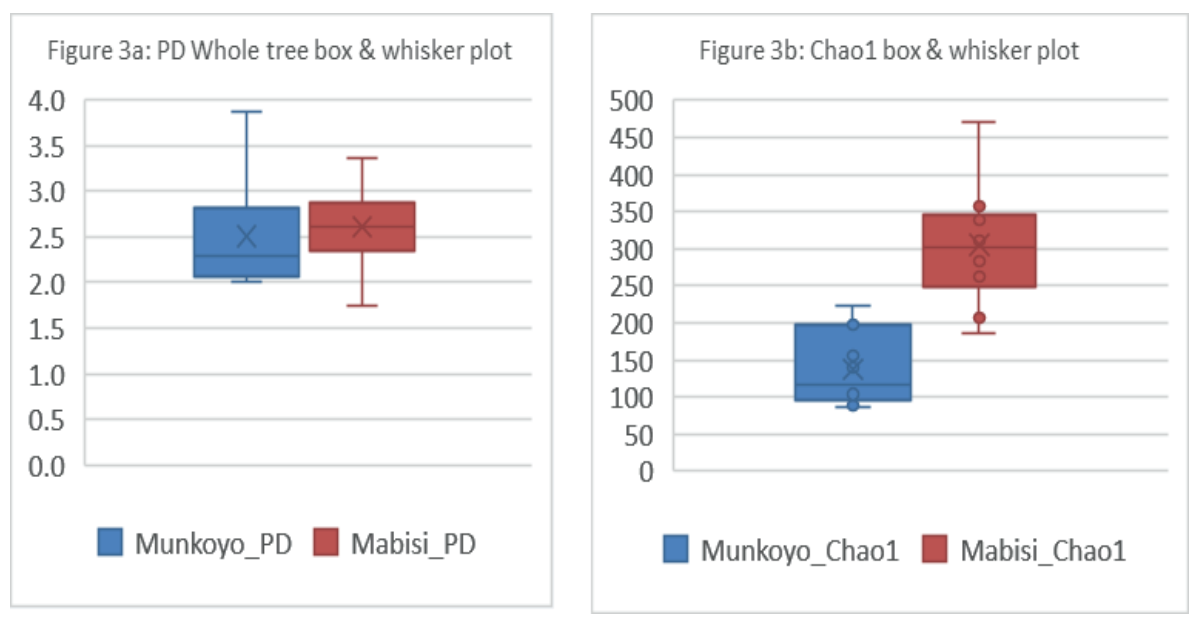

Figure 3: Comparison of alpha diversity indices of Mabisi and Munkoyo samples based on Faith's phylogenetic diversity measure (PD) and Chao1. The blue box plots indicate Munkoyo and red box plots indicate Mabisi. Figure 3a shows Faith's Phylogenetic diversity index of alpha diversity and Figure $3 \mathrm{~b}$ shows plots for Chao1 index of alpha diversity.

In the hierarchical cluster analysis (Table 4) assessing what variables contribute to the observed variation in microbial composition between the samples, we included two processing variables: the type of products (two categories, Mabisi and Munkoyo) and the type of fermentation vessel used (four categories). Both processing variables explained significant parts of the variation in microbial profiles. We 
further included the categorical data of the nine nutritional parameters, namely protein, fat, water soluble vitamins, and minerals. Except for vitamin B1 and vitamin B3, the categorization of nutritional variables explained significant parts of the variation in microbial community composition. Thus, our results are consistent with the notion that there is a link between microbial and nutritional composition of each sample.

Table 4. Results of the hierarchical cluster analysis using analysis of similarities (ANOSIM) for impact of various nutritional parameters, product type and fermentation vessel on microbial composition.

\begin{tabular}{lccc}
\hline \multicolumn{1}{c}{ Variable } & Anosim R & Number of Groups & P Value \\
\hline Product and processing variables & & 2 & $<0.001$ \\
Product type Mabisi or Munkoyo & 0.816 & 4 & $<0.001$ \\
Fermentation vessel used & 0.605 & 2 & \\
Nutritional variables & & 2 & $<0.001$ \\
Protein & 0.816 & 3 & $<0.001$ \\
Fat & 0.78 & 2 & 0.718 \\
B1 & -0.0683 & 3 & $<0.001$ \\
B2 & 0.532 & 2 & 0.436 \\
B3 & -0.0374 & & $<0.001$ \\
B6 & & 2 & $<0.001$ \\
B12 & 0.816 & 3 & \\
Ca & 0.772 & 2 & \\
\hline
\end{tabular}

Notes: Variable, test statistic (R), number of treatment groups (\# Of Groups) and exact $p$ value $(P)$ are given, unless the $p$ value was smaller than 0.001 , which is indicated by $<0.001$.

\section{Discussion}

The aim of this study was to characterize nutritional composition and microbial community composition of two traditional fermented foods, Mabisi that is based on raw milk and Munkoyo that is based on maize. The results in this study clearly showed that the two products were different with respect to the nutritional parameters and the microbial composition. Mabisi was found to have higher nutritional values for crude protein, fat, and carbohydrates than Munkoyo. Also microbial community composition in Mabisi samples was more abundant in Streptococcus, Enterobacter, and Lactococcus species, while in Munkoyo samples were more abundant in Lactococcus and Lactobacillus species. 
The difference in nutritional composition between Mabisi and Munkoyo can be mainly attributed to the use of milk as the raw material for Mabisi and maize as the raw material for Munkoyo. Milk is a rich animal source of protein and fat. In Zambia, fresh milk (unfermented) is rarely consumed due to high prevalence of lactose intolerance estimated at $70-90 \%$ in the Zambian population [25]. During fermentation most of the lactose, which at the beginning is an antinutritional factor, is converted into lactic acid and other compounds [16]. In the Zambian context, this makes Mabisi more nutritious than fresh milk. It was expected that Mabisi would have had a higher concentration of B-vitamins considering that it is made from milk and Munkoyo is made from maize which has low levels of most $B$ vitamins. We found however that both products are a source of B-vitamins, which could be attributed to the fermenting bacteria which have previously been shown to produce B vitamins [4]. Mabisi was higher in calcium, iron, and zinc for which regular consumption in combination with other local foods could help to increase intake of these micronutrients. This was also reflected when one serving of Mabisi for an adult woman was considered to contribute higher amounts for calcium and zinc and also vitamin B2 and protein to the estimated average requirements. It can be said therefore that Mabisi would be a good source of nutrients for inclusion in the food based dietary guidelines.

Microbial community: Our study results showed that in the samples there were two to four dominant bacterial species that were common to the respective product types as can be seen in figure 2 . The microbial communities in the products are dominated by lactic acid bacteria, whose growth not only resulted in a low $\mathrm{pH}$ increasing food safety properties and shelf-life, but are also regarded as healthy bacteria that may enable shifts in gut microbiota composition towards a more healthy composition. Mabisi had slightly higher diversity as shown by the diversity indices that we calculated. This could be attributed to the fact that raw milk contains a wider diversity in substrates, especially in that raw milk contains more protein. More complex substrates are known to support more diverse species communities [26]. Chao1 diversity index showed higher diversity in Mabisi than Munkoyo whereas Faith's phylogenetic diversity index was the same between the two products. This may be caused by the fact that the Faith's phylogenetic diversity index uses branch lengths for assigning diversity metrics, which cannot separate lactic acid bacteria with the same level of discriminatory detail [27]. The Chao1 index on the other hand is an estimator based on the abundance of species taking into account the rare species [28]. Alpha diversity index Chao1 found in Mabisi samples (ranged from 206 to 471) was higher than what Shangpling et al. (2018) found (Chao1 ranged from 90 to 138) for the Indian naturally fermented milk product [29]. However our results were comparable with what Liu Xiao-Feng et al. (2015) found for a Chinese traditional fermented goat milk (Chao1 ranged from 166 to 640, [30]).

There was a marked difference in microbial composition between Mabisi and Munkoyo in this study, which is in slight contrast with earlier work that found that Mabisi and Munkoyo samples collected from 
the same location had similar microbial communities [15]. In our study the differences between the microbial communities of the two products could be due to variations in other determinants known to affect microbial community composition, in particular the fermentation vessel used, which indeed came out as a significant factor explaining variation in microbial communities, and the level of back-slopping. It has been established that for example back-slopping which is the transfer of a small fraction of the previous product into fresh raw material [31], ensuring transfer of microbial communities underlying the fermentation helps shape microbial communities from batch to batch. In Zambia back-slopping is usually done using a calabash as a fermenting vessel which is not washed to preserve some starter cultures that is used for the next fermentation and in our study $68 \%$ of the Munkoyo producers had used the calabash and none for Mabisi. This could imply that most of the Mabisi producers in this study area rely on the spontaneous fermentation method. This could mean that indeed environment played a role in shaping the microbial composition but also fermenting substrate could play its part because of the similarities in samples of the same type which is in agreement with what has been found before $[6,7]$.

Microbes driving nutritional composition: We found a correlation between levels of contribution to EAR of various nutrients and variation in microbial community structure. This suggest that the composition of the microbial community is a determinant of the nutritional content of the products, including the levels of B-vitamins that are known to be produced by bacteria and that are added to the raw materials by fermentation. This observed correlation suggests that research to determine the levels of enrichment with B-vitamins by the microbes present in Mabisi and in Munkoyo fermentation is worthwhile. This could be done in controlled laboratory experiments using defined mixtures of bacteria isolated from Mabisi and Munkoyo and measuring levels of B-vitamins before and after proliferation of the bacteria. In the present study, we collected product samples from producers and did not perform the fermentation ourselves. The present work could not permit us to carry out baseline nutrition analysis before fermentation so that we could attribute any changes in microbial composition to the difference between baseline and after fermentation.

Conclusion: Generally, our work is of importance by the documentation of the nutritional value of two locally produced traditional foods. More specifically, our study is a formal demonstration that a locally produced fermented food, especially Mabisi, can contribute to achieving improved nutrient intake of various important macro- and micro-nutrients. For many of the locally available foods such as Mabisi and Munkoyo, nutritional data are lacking impeding their consideration for inclusion in food based dietary guidelines. Therefore, the data generated in this study will be useful for inclusion in the food-based guidelines. We however recommend more research to especially incorporate determination of the nutritional and microbial aspects in the fermentation process for the raw materials and end-products so as to link nutritional value with fermenting microbes and conduct more genomic analysis for B vitamin 
production. Further, other recent work has shown that variation in Mabisi and Munkoyo processing methods exists and that this has an impact on microbial community composition. This in turn may also impact nutritional composition. Thus, the inclusion of other production-types of Mabisi and Munkoyo than the ones used in this study is also recommended. Finally, our work could be expanded by adding measures of bioavailability of the nutrients within the diets of consumers by determining molar ratios of phytate to zinc, iron, and calcium. 


\section{References}

1. Nout MJR: Rich nutrition from the poorest - Cereal fermentations in Africa and Asia. Food Microbiology 2009, 26(7):685-692.

2. Battcock M, Azam-Ali S: Fermented fruits and vegetables. A Global Perspective United Nations Rome Fao Agricultural Services Bulletin 1998:10-15.

3. Tamang JP, Shin D-H, Jung S-J, Chae S-W: Functional Properties of Microorganisms in Fermented Foods. Frontiers in Microbiology 2016, 7(578).

4. LeBlanc JG, Milani C, de Giori GS, Sesma F, van Sinderen D, Ventura M: Bacteria as vitamin suppliers to their host: a gut microbiota perspective. Current opinion in biotechnology 2013, 24(2):160-168.

5. Hotz C, Gibson RS: Traditional food-processing and preparation practices to enhance the bioavailability of micronutrients in plant-based diets. The Journal of nutrition 2007, 137(4):1097-1100.

6. Agyei D, Owusu-Kwarteng J, Akabanda F, Akomea-Frempong S: Indigenous African fermented dairy products: Processing technology, microbiology and health benefits. Crit Rev Food Sci Nutr 2019:1-16.

7. Abriouel H, Pérez Montoro B, Casimiro-Soriguer CS, Pérez Pulido AJ, Knapp CW, Caballero Gómez N, Castillo-Gutiérrez S, Estudillo-Martínez MD, Gálvez A, Benomar N: Insight into Potential Probiotic Markers Predicted in Lactobacillus pentosus MP-10 Genome Sequence. Front Microbiol 2017, 8:891-891.

8. Linares DM, Gómez C, Renes E, Fresno JM, Tornadijo ME, Ross RP, Stanton C: Lactic Acid Bacteria and Bifidobacteria with Potential to Design Natural Biofunctional Health-Promoting Dairy Foods. Front Microbiol 2017, 8(846).

9. Saez-Lara MJ, Gomez-Llorente C, Plaza-Diaz J, Gil A: The role of probiotic lactic acid bacteria and bifidobacteria in the prevention and treatment of inflammatory bowel disease and other related diseases: a systematic review of randomized human clinical trials. Biomed Res Int 2015, 2015:505878.

10. Kapila S, Sinha PR, Singh S: Influence of feeding fermented milk and non-fermented milk containing Lactobacillus casei on immune response in mice. Food and Agricultural Immunology 2007, 18(1):75-82.

11. Ansorena D, Astiasarán I: Fermented Foods: Composition and Health effects. 2016.

12. Ceapa C, Wopereis H, Rezaiki L, Kleerebezem M, Knol J, Oozeer R: Influence of fermented milk products, prebiotics and probiotics on microbiota composition and health. Best Pract Res Clin Gastroenterol 2013, 27(1):139-155.

13. Adam AC, Rubio-Texeira M, Polaina J: Lactose: The Milk Sugar from a Biotechnological Perspective. Critical Reviews in Food Science and Nutrition 2005, 44(7-8):553-557.

14. Moonga HB, Schoustra SE, Linnemann AR, Kuntashula E, Shindano J, Smid EJ: The art of mabisi production: A traditional fermented milk. PLOS ONE 2019, 14(3):e0213541.

15. Schoustra SE, Kasase C, Toarta C, Kassen R, Poulain AJ: Microbial Community Structure of Three Traditional Zambian Fermented Products: Mabisi, Chibwantu and Munkoyo. PLOS ONE 2013, 8(5):e63948.

16. Justin Chileshe EFT, Sijmen E Schoustra, Karin J Borgonjen-van den Berg, Ray Handema, Bas J Zwaan and Inge D Brouwer: Potential contribution of cereal and milk based fermented foods to dietary nutrient intake of 1-5 years old children in Central province in Zambia. 2019. 
17. Phiri S, Schoustra SE, Van den Heuvel J, Smid EJ, Shindano J, Linnemann AR: Fermented cereal-based Munkoyo beverage: processing practices, microbial diversity and aroma compounds. PLOS ONE 2019, submitted.

18. Foma RK, Destain J, Mobinzo PK, Kayisu K, Thonart P: Study of physicochemical parameters and spontaneous fermentation during traditional production of munkoyo, an indigenous beverage produced in Democratic Republic of Congo. Food Control 2012, 25(1):334-341.

19. Groenenboom AE: Microbial community dynamics in traditionally fermented milk. Wageningen: Wageningen University; 2019.

20. AOAC: Official Methods of Analysis of the Association of Official Analytical Chemists, 17th Edition, Washington Dc. 2000.

21. Jorhem L: Determination of metals in foods by atomic absorption spectrometry after dry ashing: NMKL Collaborative Study. Journal of AOAC International 2000, 83(5):1204-1211.

22. Zafra-Gómez A, Garballo A, Morales JC, García-Ayuso LE: Simultaneous Determination of Eight WaterSoluble Vitamins in Supplemented Foods by Liquid Chromatography. Journal of Agricultural and Food Chemistry 2006, 54(13):4531-4536.

23. Moreno $P$, Salvadó V: Determination of eight water- and fat-soluble vitamins in multi-vitamin pharmaceutical formulations by high-performance liquid chromatography. Journal of Chromatography $A$ 2000, 870(1):207-215.

24. WHO: Guidelines on food fortification with micronutrients. 2006.

25. de Vrese M, Stegelmann A, Richter B, Fenselau S, Laue C, Schrezenmeir J: Probiotics-compensation for lactase insufficiency. The American Journal of Clinical Nutrition 2001, 73(2):421s-429s.

26. Hardin G: The competitive exclusion principle. Science (New York, NY) 1960, 131(3409):1292-1297.

27. Faith DP: Conservation evaluation and phylogenetic diversity. Biological Conservation 1992, 61(1):1-10.

28. Chao A, Chiu C-H, Jost L: Phylogenetic diversity measures based on Hill numbers. Philosophical Transactions of the Royal Society B: Biological Sciences 2010, 365(1558):3599-3609.

29. Shangpliang HNJ, Rai R, Keisam S, Jeyaram K, Tamang JP: Bacterial community in naturally fermented milk products of Arunachal Pradesh and Sikkim of India analysed by high-throughput amplicon sequencing. Scientific reports 2018, 8(1):1532.

30. Liu X-F, Liu C-J, Zhang H-Y, Gong F-M, Luo Y-Y, Li X-R: The bacterial community structure of yond bap, a traditional fermented goat milk product, from distinct Chinese regions. Dairy Science \& Technology 2015, 95(3):369-380.

31. Nout MJR, Rombouts FM: Fermentative preservation of plant foods. Journal of Applied Bacteriology 1992, 73(s21):136s-147s.

32. Kanehisa M, Goto S: KEGG: kyoto encyclopedia of genes and genomes. Nucleic acids research 2000, 28(1):27-30.

33. Authority EFS: Dietary Reference Values for nutrients Summary report. EFSA Supporting Publications 2017, 14(12):e15121E. 


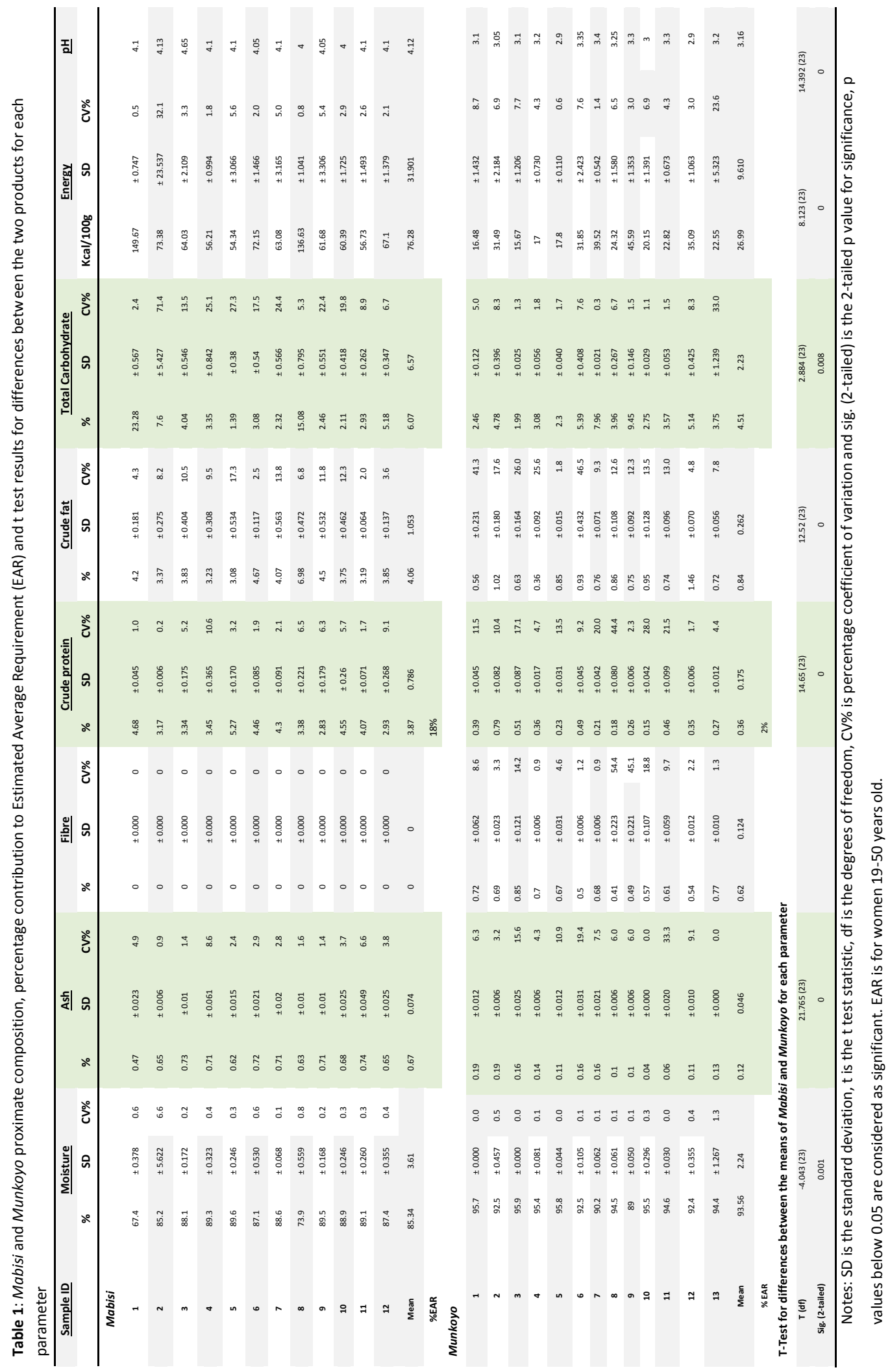




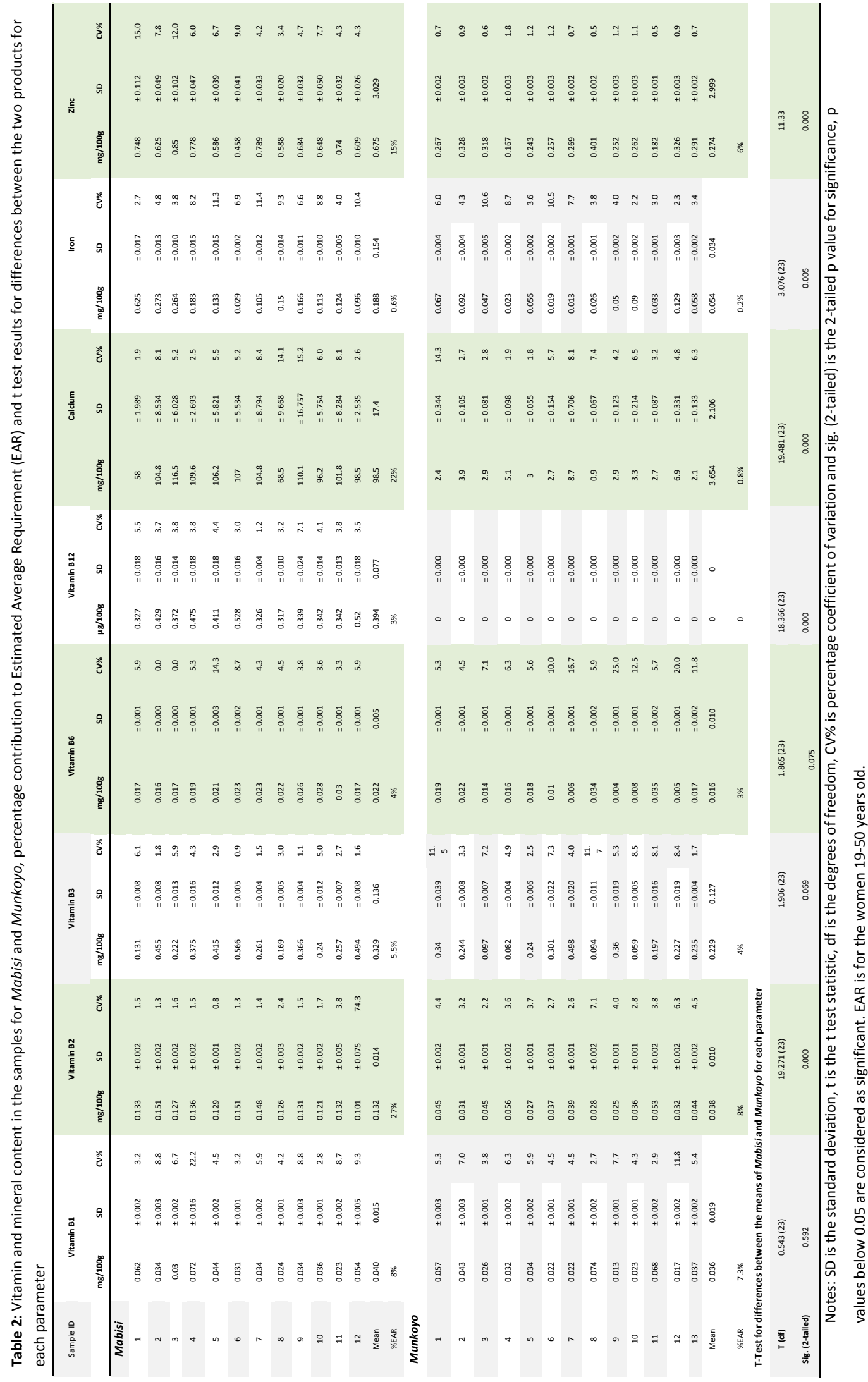




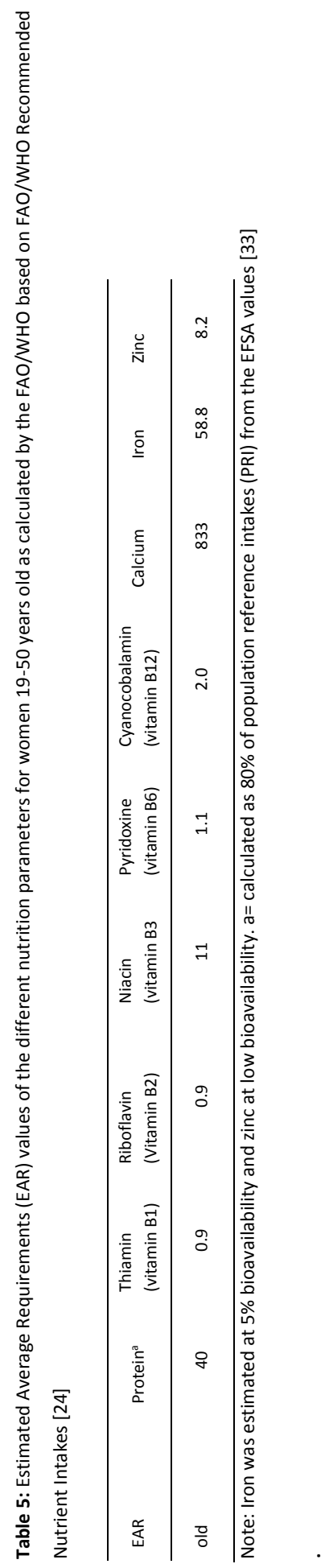


Supplementary Table 6: Variation accounted for by the different nutritional parameters (in rotated space by Varimax with Kaiser Normalization) ${ }^{a}$. Principal Components 1 and 2 are shown with loadings of the nutritional parameters contribution to the respective components. Components 2 variation in the samples is explained by vitamins B1 and B6. Explain that loadings have a value between -1 and +1 , zero being the average of all observations.

\begin{tabular}{lll}
\hline Rotated Component Loadings ${ }^{\mathbf{a}}$ & & \\
\hline & \multicolumn{1}{l}{ Components } & \\
\cline { 2 - 2 } & $\mathbf{1}$ & \\
Ash & 0.957 & \\
B12 & 0.944 & \\
Crude fat & 0.944 & \\
B2 & 0.942 & \\
pH & 0.941 & \\
Energy & 0.936 & \\
Ca & 0.936 & \\
Crude protein & 0.921 & \\
Zn & 0.911 & \\
Moisture & -0.813 & -0.283 \\
Total Carbohydrate & 0.762 & 0.875 \\
Fe & 0.708 & \\
B3 & 0.353 & \\
B6 & & \\
B1 & & \\
\hline
\end{tabular}

Variable Principal Normalization.

a. Rotation Method: Varimax with Kaiser Normalization. 
Chapter 4 
Do traditional fermented foods affect the gut microbiota metabolism? Assessing the potential using an in vitro system

Justin Chileshe ${ }^{1,2}$, Jay Sikalima ${ }^{1}$, Phidelis Malunga ${ }^{1}$, Ray Handema ${ }^{1}$, Bas Zwaan ${ }^{2}$, Elise Talsma ${ }^{3}$ and Sijmen E. Schoustra ${ }^{2,4}$

1. Tropical Diseases Research Centre, Biomedical Sciences Department, Ndola, Zambia

2. Wageningen University, Laboratory of Genetics, Wageningen, Netherlands

3. Wageningen University, Division for Human Nutrition and Health, the Netherlands

4. The University of Zambia, School of Agricultural Sciences, Department of Food Science and Nutrition, Lusaka, Zambia 


\section{Abstract}

The microbial composition of the human gut differs between healthy and less-healthy individuals and can be said to influences human health. The human gut microbiota is affected by diet, with fermented foods having been found beneficial in modulating the gut microbiota. Several foods naturally contain lactic acid bacteria that could have probiotic effects which can be said about traditionally fermented food products that contain a microbial community consisting of a variety of lactic acid bacteria. Here we used an in-vitro single-batch culture fermentation system to study probiotic effects of traditional fermented products Mabisi and Munkoyo. We exposed stool samples to these products and various controls and measured concentrations of short chain fatty acids (SCFAs) and used these as an indicator of beneficial bacteria activity and measured shifts in levels of the beneficial bacterium Lactobacillus. Results show that exposure to any of the fermented foods results in higher levels of SCFAs and also higher levels of Lactobacillus. These results support the idea that consumption of fermented foods can result in healthier metabolism of the gut microbiota as measured with SCFA concentrations. These results can inform further more complex in-vitro as well as in vivo studies on the effects of the traditional fermented foods on gut microbiota metabolism and composition.

\section{Introduction}

The microbial composition of the human gut influences human health. Previous research has shown that gastrointestinal microflora differs between healthy and less-healthy individuals [1], with healthy individuals harboring higher levels especially of various groups of lactic acid bacteria. The microflora that is specifically present in healthy individuals were named probiotics. In subsequent research, several individual microbes have been tested and their health-promoting effect was formally shown in humans and animals. The use of probiotics is considered especially beneficial for individuals with low health status [2].

Probiotics have been defined by the World Health Organization (WHO) as "live microorganisms which when administered in adequate amounts confer a health benefit on the host" [3]. Several foods naturally contain lactic acid bacteria and these could have probiotic effects. This is especially true for traditionally fermented food products that contain a microbial community consisting of a variety of lactic acid bacteria. Recent research on lactic acid bacteria from fermented dairy products have suggested that these products contain lactic acid bacteria with probiotic properties for their consumers [4-23]. These studies suggest that probiotic effects mainly come from the consumption of viable bacteria that then alter the bacterial ecology in the gastrointestinal (GI) tract to more favorable composition [24-28]. 
In many countries various traditional fermented food products exist. Most of these products rely on the spontaneous and uncontrolled fermentation by lactic acid bacteria to produce sour products from various raw materials, such as milk and cereal [29]. Examples of such products are milk-based Mabisi and cereal based Munkoyo from Zambia. These products are widely produced and consumed by large proportions of the population including women and small children. Similar to other traditionally fermented products, Mabisi and Munkoyo consist of a mixed community of fermenting lactic acid bacteria. Bacterial communities typically contain around six to ten species present at an abundance of at least $2 \%$ and up to ten additional detectable species at lower abundance levels. Given the wide diversity of lactic acid bacteria, we expect that Mabisi and Munkoyo contain (uncharacterized) probiotic strains and that their consumption will exert probiotic effects to consumers [30-32].

Very few direct experimental tests exist of probiotic properties of bacterial consortia in fermented foods due to the complexity of such experiments. Ideally, these tests would include nutrition intervention studies comprised of feeding trials with healthy and less healthy individuals consuming (or not) these traditional foods and measurements of health parameters as well as characterization of gastrointestinal microflora [33-35]. The use of in-vitro batch culture systems has proven to be a useful proxy for these experiments in order to generate first insights into the potential probiotic properties of either bacterial strains, products, or dietary fibers [36]. In these experiments, gut microbiota obtained from stool samples is exposed to various additions of either bacterial strains or dietary fiber while monitoring shifts in metabolite profiles (e.g. concentrations of short chain fatty acids, SCFAs) as well as shifts in microbial composition. As such, these experiments have generated insight in what bacteria or compounds likely have probiotic effects on intestinal bacteria [36-38]. Metabolites such as SCFAs are monitored in such experiments because of their importance as products of microbial fermentation of dietary fibre in human large intestines. These levels of SCFAs are considered proxies for health benefits to the host since they play an important role in modulating the health of the host through a range of tissue-specific mechanisms related to gut barrier function, glucose homeostasis, immunomodulation, appetite regulation and obesity among other functions [39].

Taking inspiration from these studies, we used an in vitro model to study probiotic effects of Mabisi and Munkoyo. Specifically we asked if, (1) there are differences in concentrations of SCFAs as an indicator of beneficial bacteria activity when exposing the microbes in the stool samples to Mabisi or Munkoyo and, (2) if exposure of stool microbiota to Mabisi and Munkoyo shifts the levels of the indicator beneficial bacterium Lactobacillus. We used an experimental approach, adding two different amounts (high and low) of Mabisi and of Munkoyo to stool samples. As controls we included a treatment with sterilized Mabisi and Munkoyo that did not contain live bacteria, a fructo-oligo saccharides (FOS) as positive control, and only stool or only product as negative controls. We expected 
that addition of either Mabisi or Munkoyo would lead to increased levels of SCFAs and a higher abundance of Lactobacillus, both of which are indicators of activity of beneficial bacteria among the gut microbiota in the stool samples.

\section{Methods}

We used an in-vitro single-batch culture fermentation system following the procedure described by Rösch et al with some modifications [40]. This was system simulates the human colon, and for our experiment we simulated ileal environment medium (SIEM).

Preparation of SIEM: The media components (BCO, Salts, MgSO4, Cysteine. $\mathrm{HCl}$, Vitamin mix) were purchased from Tritium Microbiologie, the Netherlands. MES buffer was prepared using 2-(Nmorpholino) ethanesulfonic acid purchased from Sigma Aldrich, The Netherlands. The full SIEM composition is shown in Table 1.

Table 1: Composition of the simulated intestinal environment medium.

\begin{tabular}{|c|c|c|}
\hline Components & & g/I SIEM \\
\hline & Bactopepton & 24 \\
\hline \multirow[t]{4}{*}{ BCO } & Casein & 24 \\
\hline & Ox-bile & 0.4 \\
\hline & $\mathrm{K} 2 \mathrm{HPO} 4.3 \mathrm{H} 2 \mathrm{O}$ & 2.5 \\
\hline & $\mathrm{NaCl}$ & 4.5 \\
\hline \multirow[t]{3}{*}{ Salts } & $\mathrm{CaCl} 2.2 \mathrm{H} 2 \mathrm{O}$ & 0.45 \\
\hline & $\mathrm{FeSO} 4.7 \mathrm{H} 2 \mathrm{O}$ & 0.005 \\
\hline & Haemine & 0.01 \\
\hline MgSO4 & & 0.4 \\
\hline \multirow[t]{4}{*}{ Cystein.HCl } & & 0.16 \\
\hline & Menadion & 1 \\
\hline & Biotin & 2 \\
\hline & vitamin B12 & 0.5 \\
\hline \multicolumn{3}{|l|}{ Vitamin mix } \\
\hline & pantothenate & 10 \\
\hline & nicotinaminde & 5 \\
\hline & para-aminobenzoic acid & 5 \\
\hline
\end{tabular}


All the media components were mixed together in a Media Glass Bottle except vitamin mix, cysteine, and MES buffer and were boiled for 20 seconds. The mixture was cooled down under an oxygen-free $\mathrm{N}_{2}$ flow, then cysteine was added and the mix was transferred into the $50 \mathrm{ml}$ serum bottles. The serum bottles were closed with rubber stoppers and aluminum caps and were flushed with the gas mixture $\left(95 \% \mathrm{~N}_{2}\right.$ and $\left.5 \% \mathrm{H}_{2}\right)$. The media was autoclaved after which vitamin mix and MES buffer was added using a sterile syringe and needle. MES buffer ( $1 \mathrm{M} \mathrm{pH} \mathrm{6.0)}$ was prepared by dissolving $50 \mathrm{~g}$ of 2-(Nmorpholino) ethanesulfonic acid in $200 \mathrm{ml}$ of distilled water to make a $1 \mathrm{M}$ solution and was filtered through a $0.2 \mu \mathrm{m}$ filter into the sterile bottle.

Collection and treatment of stool samples: Stool samples were collected from three healthy Zambian adults volunteers (two males and one female) who had not been treated with antibiotics one month prior to sampling. Informed consent was obtained and the study explained to the volunteers before collection of the stool samples as per ethical requirements. This study was approved by the Tropical Diseases Research Centre (TDRC) Ethics review committee (Ndola, Zambia) (Ethics number $\mathrm{STC} / 2015 / 13)$. After collection, $10 \%(\mathrm{w} / \mathrm{v})$ faecal slurry was prepared by homogenizing $5 \mathrm{~g}$ of faeces in $50 \mathrm{~mL}$ of $0.1 \mathrm{M}$ Phosphate buffer. The samples were flushed through a filter using gas mixture of $95 \%$ $\mathrm{N}_{2}$ and $5 \% \mathrm{H}_{2}$ and were stored at $4^{\circ} \mathrm{C}$.

Mabisi and Munkoyo samples used in the fermentation experiment were collected from the local producers in Mkushi. The sterilized Mabisi and Munkoyo samples were prepared by exposure under UV-light for 24 hours to kill all the bacteria. The samples were plated on Plate Count Agar (PCA) and MRS agar to see if there were any viable bacteria; nothing grew for the period of 72 hours. Fructooligosaccharide (FOS) purchased from Sigma Aldrich was used as positive control.

\section{Preparation and inoculation of stool in medium Mabisi/Munkoyo and positive, negative samples} inoculation.

The fermentation reaction comprised of $29.325 \mathrm{ml}$ of SIEM media, Treatment (FOS, Mabisi, Munkoyo or sterilized Mabisi/Munkoyo) of $0.3 \mathrm{ml}(1 \%)$ and faecal Solution volume of $0.375 \mathrm{ml}$ (1.25\%) giving a total reaction volume of $30 \mathrm{ml}$ as in Table 2 . The fermentation reactions were performed in triplicate and incubated at $37^{\circ} \mathrm{C}$. Sampling was done in triplicate, each of $1 \mathrm{ml}$, using a sterile needle and syringe at time points $0,12,24,48$ and 72 hours. All samples were stored at $-20^{\circ} \mathrm{C}$ until analysis. The fermentation reactions were performed with different treatments including Mabisi High 
concentration, Mabisi Low concentration, Munkoyo High concentration and Munkoyo Low concentration. Fructo-oligo-saccharide (FOS) was included as a positive control since this sugar is known for its positive effect towards higher levels of SCFAs and as negative controls we had included treatments with only stool and with only product (Mabisi or Munkoyo). The same levels of FOS or product only were added in each of the Mabisi high/low and Munkoyo high/low treatments, so these could be regarded as replicated experiments or blocks.

\section{Experimental setup: table with treatments}

Table 2: Experimental setup for the in vitro fermentation. The experiment was repeated in three biological replicates using three independent stool samples.

\begin{tabular}{|c|c|c|c|}
\hline Sample & $\begin{array}{l}\text { Product or FOS } \\
(\% \mathrm{w} / \mathrm{v})\end{array}$ & $\begin{array}{l}\text { Feacal matter } \\
(\% \mathrm{w} / \mathrm{v})\end{array}$ & $\begin{array}{l}\text { Total volume } \\
\text { medium (ml) }\end{array}$ \\
\hline Mabisi or Munkoyo & 4 (high) or 1 (low) & 1.25 & 30 \\
\hline Sterilized & 1 & 1.25 & 30 \\
\hline \multicolumn{4}{|l|}{ Mabisi/Munkoyo } \\
\hline FOS & 1 & 1.25 & 30 \\
\hline Faeces only & - & 1.25 & 30 \\
\hline Mabisi only & 1 & - & 30 \\
\hline Munkoyo only & 1 & - & 30 \\
\hline
\end{tabular}

\section{Analysis of Short Chain Fatty Acids (SCFAs) by HPLC}

Acetate, propionate, butyrate, lactate, and isobutyrate were measured in duplicate for each sample using a SHIMADZU Prominence HCT2010 high performance liquid chromatograph (HPLC) according to the procedure described by De Baere et al with modifications [41]. The HPLC system was equipped with an Hypersil Gold aQ $(150 \mathrm{~mm} \times 4.6 \mathrm{~mm}$, dp: $3 \mu \mathrm{m})$ column (Thermal Fischer Scientific, cat \#: 25303154630) and a Shimadzu photo diode array detector and operated at $65^{\circ} \mathrm{C}$ using $5 \mathrm{mM} \mathrm{H}_{2} \mathrm{SO}_{4}$ as the mobile phase at a flow rate of $0.6 \mathrm{~mL} / \mathrm{min}$. External standard calibration curves were constructed for each of the SCFAs and succinate was used as an internal standard to calculate the extraction efficiency.

Quality control: A pooled sample was made as a control sample. This control sample was included in duplicate for every batch of samples on a daily basis and the mean, standard deviation and coefficient of variation percent were calculated. The results were accepted on the basis of the \%CV being less than $10 \%$ for a particular batch of samples. 


\section{Determination of bacterial composition in stool samples}

\section{Sample DNA isolation:}

Whole genomic DNA from each fermentation sample was prepared by taking a $200 \mu \mathrm{l}$ aliquot into a sterile bead-beating tube containing $300 \mathrm{mg}$ of glass beads (diameter $0.1 \mathrm{~mm}$ ) using the Zymo Research Quick DNA faecal/soil microbe microprep kit (Cat \# 6010 and 6012) for DNA isolation in stool samples after bead-beating in a Sunon mini bead-beater according to manufacturer's instructions.

\section{Abundance of Lactobacillus (Quantitative PCR)}

A method adapted from Takagi et al. was used for this analysis targeting All Eubacteria, and Lactobacillus [37]. Real-time quantitative PCR was performed with the Applied Biosystems AB7500 Real Time thermocycler. The primer sets described in the Table 3 below were used for measuring the partial 16S rRNA gene copy number. The quantitative measurement by real-time PCR was conducted in triplicate. The real-time PCR amplification program for eubacteria was as follows: $95^{\circ} \mathrm{C}$ for $3 \mathrm{~min}$, followed by $38 \mathrm{cycles}$ of $95^{\circ} \mathrm{C}$ for $30 \mathrm{sec}$ and $54^{\circ} \mathrm{C}$ for $30 \mathrm{sec}$; for Lactobacillus: $50^{\circ} \mathrm{C}$ for $2 \mathrm{~min}, 95^{\circ} \mathrm{C}$ for $10 \mathrm{~min}$ followed by 38 cycles of $95^{\circ} \mathrm{C}$ at $15 \mathrm{sec} \& 60^{\circ} \mathrm{C}$ for $1 \mathrm{~min}$ ). To check the specificity of the amplifications, a melting curve was obtained by performing the following cycle: a denaturation step at $95^{\circ} \mathrm{C}$ for $15 \mathrm{sec}$, a $1^{\circ} \mathrm{C}$ increase in temperature every $20 \mathrm{sec}$ starting at $60^{\circ} \mathrm{C}$ and ending at $95^{\circ} \mathrm{C}$, and a final step at $95^{\circ} \mathrm{C}$ for $15 \mathrm{sec}$. Standard curves for absolute quantification in the cultures were prepared using 102-106 copies of the PCR fragments of the 16S rRNA genes. The correlation coefficients for all the standard curves exceeded 0.99. For each assay, $2 \mu \mathrm{L}$ of DNA solution was added to $18 \mu \mathrm{L}$ of a PCR mixture containing $10 \mu \mathrm{L}$ of THUNDERBIRD ${ }^{\text {TM }}$ SYBR1 qPCR Mix (Applied Biosystems), 7.2 $\mu \mathrm{L}$ of distilled water, and $200 \mathrm{nM}$ of each primer.

Table 3: Primer sets used for qPCR and amplification parameters for All Eubacteria and Lactobacillus.

\begin{tabular}{|c|c|c|c|}
\hline Name & Primer Sequences & $\begin{array}{l}\text { qPCR amplification } \\
\text { parameters }\end{array}$ & Melt curve \\
\hline All Eubacteria & $\begin{array}{l}\text { F: ACTCCTACGGGAGGCAGCAGT } \\
\text { R: GTATTACCGCGGCTGCTGGCAC }\end{array}$ & $\begin{array}{l}95^{\circ} \mathrm{C}-3 \mathrm{~min}, 38 \mathrm{cycles} \text { of } \\
\left(95^{\circ} \mathrm{C}-30 \mathrm{sec} \& 54^{\circ} \mathrm{C}-30 \mathrm{sec}\right)\end{array}$ & $\begin{array}{l}95-15 \mathrm{sec}, 1 \% \text { decrease } 20 \mathrm{sec} \\
\text { starting at } 60^{\circ} \mathrm{C} \text { ending at } 95^{\circ} \mathrm{C} \text {. } \\
\text { final step at } 95^{\circ} \mathrm{C}-15 \mathrm{sec}\end{array}$ \\
\hline Lactobacillus group & F: AGCAGTAGGGAATCTTCCA & $\begin{array}{l}50^{\circ} \mathrm{C}-2 \mathrm{~min}, 95^{\circ} \mathrm{C}-10 \mathrm{~min}, \\
38 \mathrm{cycles} \text { of }\left(95^{\circ} \mathrm{C}-15 \mathrm{sec} \&\right. \\
\left.60^{\circ} \mathrm{C}-1 \mathrm{~min}\right)\end{array}$ & $\begin{array}{l}95-15 \mathrm{sec}, 1 \% \text { decrease } 20 \mathrm{sec} \\
\text { starting at } 60^{\circ} \mathrm{C} \text { ending at } 95^{\circ} \mathrm{C} \text { - } \\
\text { 30sec. final step at } 60^{\circ} \mathrm{C} 15 \mathrm{sec}\end{array}$ \\
\hline
\end{tabular}




\section{Statistical analysis}

We exposed stool of three healthy individuals to one of two traditional fermented products, milkbased Mabisi and cereal-based Munkoyo, using a high and a low level of product in separate experiments. We further included various control treatments. The different stool samples were treated as biological replicates. Each biological replicate consisted of three technical replicates. After initiating the exposure experiments, we sampled at five time points over 72 hours. For each of the samples, we measured levels of five short chain fatty acids (Lactic acid, acetic acid, propionic acid, butyric acid and isobutyric acid) and we quantified the abundance of Lactobacillus among the stool microbiota.

The output data set including $\mathrm{Ct}$ number, bacteria name, sample name, concentration and replicate was opened directly in Microsoft ${ }^{\circledR}$ Excel from the exported $\mathrm{Ct}$ file from the AB $7500 \mathrm{qPCR}$ machine and then data was exported to SPSS for processing. Data from HPLC analysis of the Short-Chain Fatty acids was also added. Student $t$ test, Pearson's correlation test and repeated measures multivariate analysis of variance (MANOVA) were performed to show the trends over time and differences between treatments and between the biological replicates. The repeated measures MANOVA was performed using the dependent variable defined as either the combined SCFAs or combined abundance of bacteria and measured against the independent variables including time point, treatment and the biological replicates. Post hoc tests were performed to compare the differences between the biological replicates (Mabisi or Munkoyo, FOS, Sterilised Mabisi or Munkoyo, No stool and No product). The biological replicate (no product) had everything included except the product (Mabisi or Munkoyo).

\section{Results}

Results of measurements of short chain fatty acids (SCFA) are shown in Figure 1 and abundance of Lactobacillus are shown in Figure 2, with the details of the statistical tests are reflected in Table 2. Results show that addition of FOS leads to higher levels of SCFA than the controls. While quantitative differences between treatments exist, this general trend is qualitatively true for all SCFA measured and for all treatments.

The statistical analyses presented in Table 4 and supplementary Table 7 and results in Figure 1 show that the addition of Mabisi had an effect on the concentrations of SCFA over time. Levels of SCFA approach the levels of SCFA in the positive control (FOS) treatment, thus showing a clear positive effect of Mabisi on SCFA production. When comparing the high and low-level of Mabisi addition, results showed that for the low level treatment the increase in SCFA is delayed relative to when adding high levels of Mabisi, however, this difference is not statistically significant (Table 4). When adding high 
levels of Mabisi, the main dynamics of increase occur during the first 24 hours, when adding low levels of Mabisi dynamical changes occur up to the end of the experiment (72 hours). Final levels of SCFA remain slightly lower after low level Mabisi addition Table4 and supplementary Table 7. The addition of sterilized Mabisi at either high or low levels leads to a slight increase in SCFA that is not statically significant (Figure 1), overall this increase is higher than the increase observed in the negative controls. However sterilized Mabisi leads to less increase in SCFA than the untreated product.

While the effects are not statistically significant (Figure 1; Table 4), the addition of Munkoyo result in the same qualitative results on SCFA concentrations as the addition of Mabisi. The addition of Munkoyo leads to SCFA levels that are similar to the positive control (FOS). Absolute levels of SCFA as well SFCA levels relative to the negative control are lower overall than the Mabisi experiments. This is attributable to a block effect of carrying out two sets of experiments. Some of the measurements of SCFA in the low Munkoyo series has failed due to technical limitations.

We measured the relative abundance of Lactobacillus as fraction of all eubacteria for all of the treatments for each of the samples. The abundance of Lactobacillus was higher for the Mabisi treatments than Munkoyo treatments as shown in Figure 2. Results are similar to those for SCFAs and show that abundance of Lactobacillus is highest after addition of FOS and product and lowest for the negative control of no product. Any increase in abundance of Lactobacillus in the control treatment of only product is due to the growth in the SIEM medium by Lactobacillus strains originating from the product and was made as a reference for comparisons with the other biological replicates (Mabisi/Munkoyo, FOS and sterile Mabisi/Munkoyo).

\section{Repeated measures Multivariate analysis of variance (MANOVA).}

The repeated measures MANOVA revealed that there were differences between the within Mabisi and Munkoyo as well as between Mabisi and Munkoyo treatments for both the SCFAs and lactobacillus as shown in Table 4. It is shown that there was a statistically significant difference for the SCFAs (Pillai's Trace $=0.555, F(4,120)=1.279, p=0.026)$ and not for bacteria (Pillai's Trace $=0.092, F(1,32)=$ $0.807, p=0.763$ ) at different time points for the biological replicate in all the treatments. Tukey's HSD post hoc test was applied and revealed that there was no difference between Mabisi and FOS for all the other biological replicates but there was a statistically significant difference between Mabisi and the other biological replicates as well as between FOS and the other replicates as shown in the supplementary Table 7. The increasing trend is seen from the results of multiple comparisons between the biological replicates in the supplementary Table 7 with the positive mean differences indicating an increase or negative mean difference value indicating a decrease. From the post hoc results, the 
negative control ("no product") replicate was made a reference and plotted against the other biological replicates to determine which ones were statistically different from the reference. In Figure 1 panels 1 and 2, Mabisi and FOS were statistically significantly higher than the reference while the sterile Mabisi and replicate with no stool were not. Figure 1 panels 3 and 4 showed that Munkoyo and FOS were higher than the reference compared to sterile Munkoyo and replicate with no stool but not statistically significant. 


\section{Panel 1 Mabisi high}

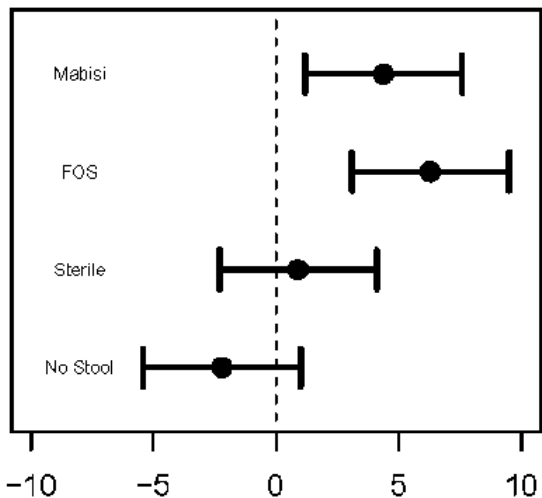

relative SCFAs

Panel 2 Mabisi low

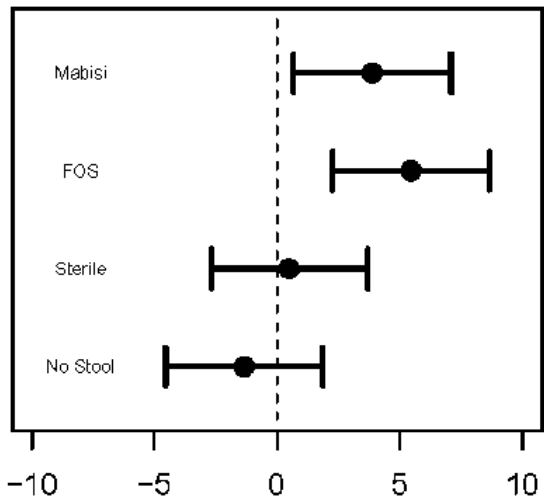

relative SCFAs

\section{Panel 3 Munkoyo high}

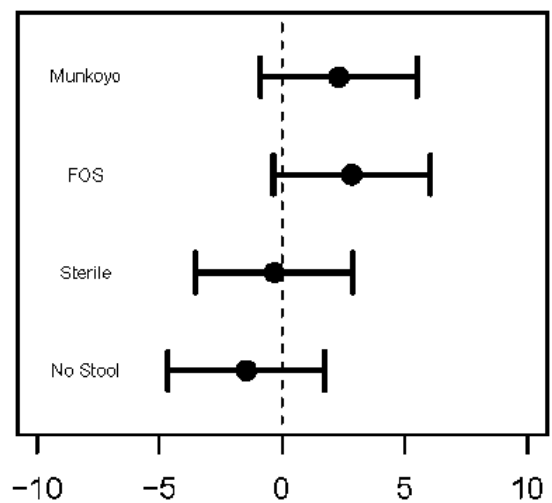

relative SCFAs

\section{Panel 4 Munkoyo low}

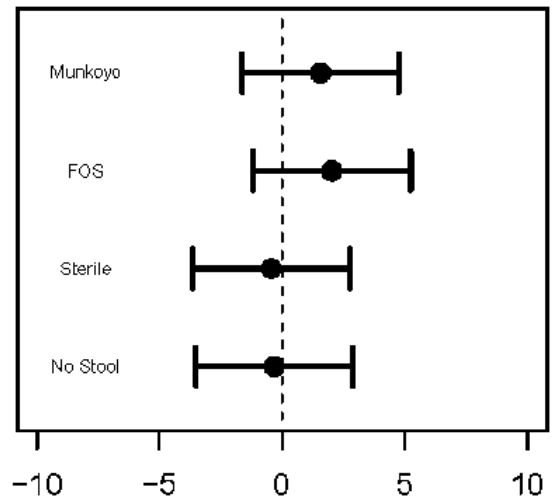

relative SCFAs

Figure 1. Mean difference in relative SCFA concentrations per level and type of product added to stool (Mabisi high, Mabisi low, Munkoyo high, Munkoyo low). Each panel shows the SCFA levels of treatments (product, FOS, sterile product, no stool/only product) relative to the negative control (only stool). Values above zero indicate higher SCFA concentrations than the control. Error show the $95 \%$ confidence interval of each average. If the error bar does not include zero, the SCFA production in that particular treatment is statistically different than the negative control. Supplementary Table 7 shows full statistical details. Supplementary Figure 2 shows plots for each SCFA per treatment and time point.

Table 4: Results of a repeated measures Multivariate analysis of variance for the SCFAs and Bacteria with Pillai's Trace test statistic. Factor1 are either SCFAs or bacteria as dependent variable. Biological replicates are Mabisi or Munkoyo or FOS or 
Sterile Mabisi/Munkoyo or No Mabisi/Munkoyo or No stool. Treatment is the High or Low levels of Mabisi or Munkoyo added to the reaction.

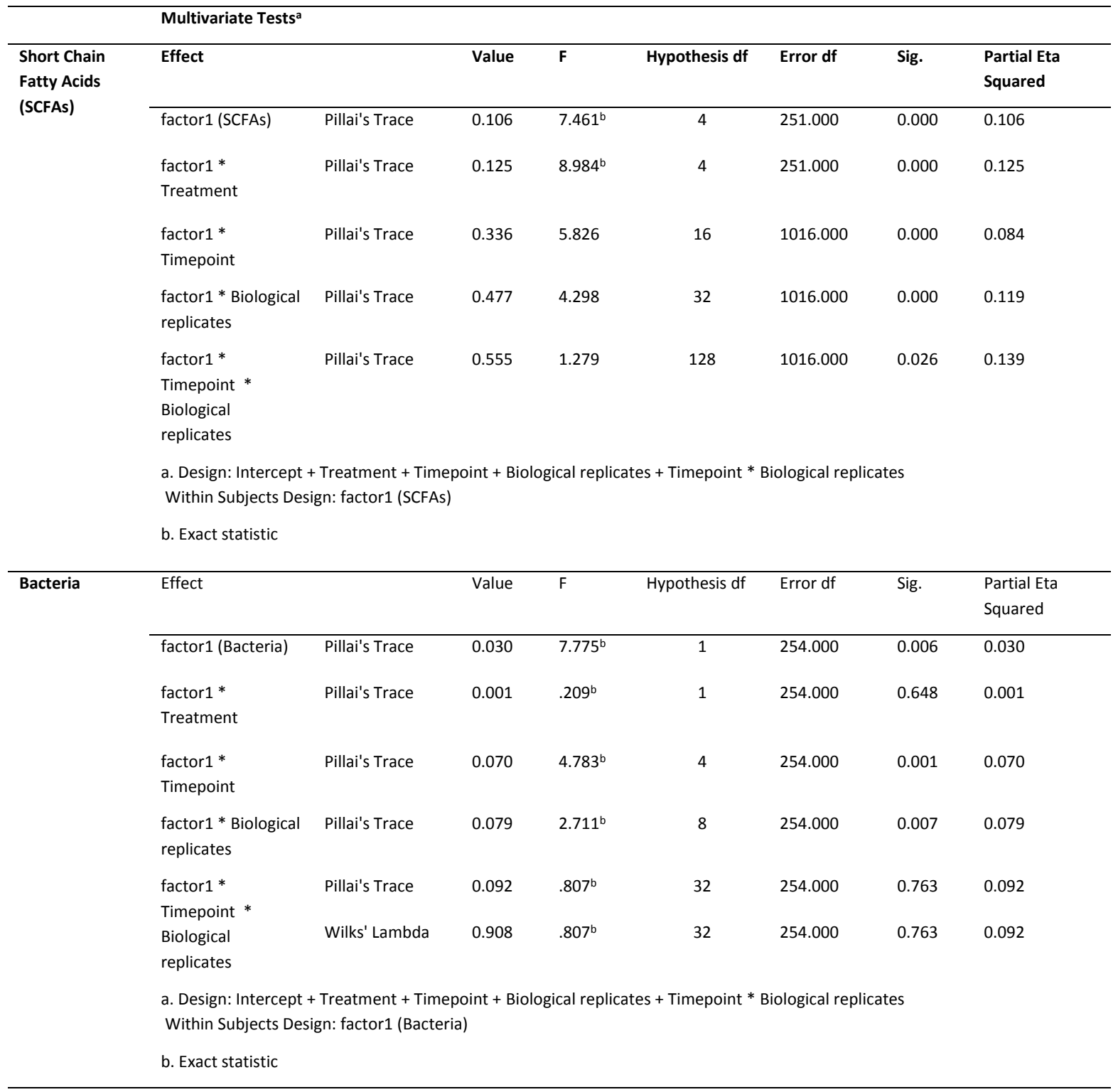

\section{Discussion}

In this study, we assessed the potential probiotic effect of traditional fermented foods on the production of SCFAs by gut bacteria. We used an in-vitro laboratory system that allows for analysis of production of metabolites (SCFAs) of bacterial consortia from gut microbiota in response to addition 
of traditional fermented foods and for monitoring of shifts in abundance of Lactobacillus. The increase in SCFAs and abundance of bacteria in the in vitro experiments are used as proxies for determining the impact of fermented foods. Our experimental design included various treatments allowing evaluation of various contrasts, which we summarize in Table 5. From the findings summarized in Table 5, some general inferences can be made. The results show that the methods worked in the experiment as we could contrast the positive control (FOS) from the negative controls that we used. We can further conclude that fermented products were found to be as effective as the positive control (FOS) in increasing the concentrations of SCFAs and therefore they can be considered beneficial through some general properties of the foods, but mostly via the interaction between the gut microbes and those in the products. In addition, levels of SCFA may have increased through the added level of resources (Mabisi is "richer" in nutrients that allow for microbial growth and metabolism than Munkoyo).

Previous studies have shown that higher levels of SCFA are a proxy for metabolism that is healthier for the human host. In our study, we focussed on concentrations of SCFA as these are indicators of the metabolism of the entire microbial consortium in the stool and we used stool as a proxy for gut microbiota in the entire gut system. The fact that upon addition of FOS there is an increase in SFCA production and the fact that this increase does not occur in the negative controls shows that our in vitro experimental set-up allows to detect effects of addition of fibres and other compounds on the metabolism of the stool derived gut microbiota. This confirms findings of other studies who have used the same in vitro approach. Given these findings, our results showing similar SCFA concentrations when adding FOS or one of the products can be interpreted that Mabisi and Munkoyo can alter the overall metabolism of the gut microbiota towards a metabolism that has benefits to the host. This conclusion is strengthened by the fact that we found a higher abundance of Lactobacillus in the microbial consortia that were exposed to the product and to FOS. Higher Lactobacillus abundance is regarded as an indicator for healthy composition of gut microbiota. 
Table 5. Contrasts within our experimental design, experimental results, and their interpretation.

\begin{tabular}{|c|c|c|}
\hline Contrast & Result & Interpretation \\
\hline $\begin{array}{l}\text { Positive control (FOS) vs controls (no } \\
\text { stool and product only) }\end{array}$ & $\begin{array}{l}\text { Addition of FOS leads to higher levels } \\
\text { of SCFA than no stool of product only }\end{array}$ & $\begin{array}{l}\text { The experimental set-up is suitable to } \\
\text { allow the detection of a beneficial } \\
\text { effect of adding probiotics }\end{array}$ \\
\hline FOS vs product (Mabisi or Munkoyo) & Levels of SCFA are similar for both & $\begin{array}{l}\text { Mabisi and Munkoyo have a probiotic } \\
\text { effect }\end{array}$ \\
\hline $\begin{array}{l}\text { Untreated product vs sterilized } \\
\text { product }\end{array}$ & $\begin{array}{l}\text { Untreated product leads to higher } \\
\text { levels of SCFA than sterilized product. } \\
\text { Sterilized product leads to higher } \\
\text { levels of SCFA than negative controls }\end{array}$ & $\begin{array}{l}\text { Some of the effect of product addition } \\
\text { may be through physicochemical } \\
\text { properties, such as low pH of the } \\
\text { product, activity of microbes from the } \\
\text { fermented product also contributes }\end{array}$ \\
\hline High vs low levels of product addition & $\begin{array}{l}\text { In both cases there is a positive effect } \\
\text { of adding the product, low levels of } \\
\text { addition lead to a delayed response }\end{array}$ & $\begin{array}{l}\text { When adding lower levels of product, } \\
\text { lower bacterial inoculum is provided, } \\
\text { leading to the delayed increase in } \\
\text { SCFA }\end{array}$ \\
\hline Mabisi vs Munkoyo & $\begin{array}{l}\text { Similar qualitative trends showing } \\
\text { that addition of each of the product } \\
\text { leads to SCFA concentrations similar } \\
\text { to those when adding the positive } \\
\text { control }\end{array}$ & $\begin{array}{l}\text { Both products have equally high } \\
\text { potential towards probiotic effects }\end{array}$ \\
\hline $\begin{array}{l}\text { Correlation between SCFA increase } \\
\text { and increase in abundance of } \\
\text { Lactobacillus }\end{array}$ & $\begin{array}{l}\text { Positive correlation, higher SFCA } \\
\text { correlates to higher abundance of } \\
\text { Lactobacillus }\end{array}$ & $\begin{array}{l}\text { Result is in line with the expectation } \\
\text { that SCFA are a result of metabolism } \\
\text { of lactic acid bacteria and that } \\
\text { addition of products leads to higher } \\
\text { Lactobacillus abundance }\end{array}$ \\
\hline
\end{tabular}

Sterilized product also had an impact resulting in higher levels of SCFA, although this was not as pronounced as the treatments where the unsterilized product was used. This shows that the probiotic effect of the product is a combination of changes in physicochemical properties of the environment and the addition of live lactic acid bacteria. The products have a low pH of around pH 4 and lowering the $\mathrm{pH}$ of the gut microbiota environment is known to promote more healthy lactic acid bacteria. Further, the products may contain secondary metabolites of the fermenting bacteria that could have an impact on the gut microbes.

A modelling study using Optifood [42] showed that Mabisi can complement current diets due to its rich nutritional content in terms of animal source protein and various micronutrients. Because 
Munkoyo contains fewer nutrients, it does not provide this direct contribution to the improvement of diets to the same extent. However, the potential probiotic effect of both products is similar, indicating that both Mabisi and Munkoyo can have a positive contribution on improved health. There are limitations to an in-vitro study that we would like to acknowledge. Our system is simplified and does not capture all aspects of the gut microbiota and the gut environment. Further, we used stool samples of three individuals that were considered to be representative, however, using other of increased number of individuals may strengthen our approach. Nevertheless our approach also had several advantages. The prime advantage is the opportunity to include various treatments and contrasts to study their potential importance for probiotic effects, such as different product types and levels of exposure, different stool donors, and comparisons with known probiotic agents such as FOS. In other words, our in-vitro approach allows for a high level of control over the many factors that may affect the health benefits of consuming fermented products. These factors can be tested singly and in combination to select the combinations that appear to have the highest potential in a randomized control or clinical test set-ups.

Thus, our findings can motivate and refine future nutrition intervention studies to assess the effect on gut microbiota and health of consumers. We explored the potential probiotic effects of two traditional fermented foods that are part of traditional diets. Adoption of these foods by potential consumers in urban areas where the product is consumed at lower frequencies may be helped by our finding that these traditional products may exert nutritional or health benefits. Based on this work, levels of consumption may not be that important as both high and low levels of exposure generated probiotic effects. The type of traditional fermented food may also not be that important, as long as it contains a wide range of live bacteria. We used healthy individuals as stool donors. It has been suggested that especially gut microbiota of less healthy individuals may benefit from probiotic interventions [2]. We may expect larger positive effects when using stool of less healthy individuals. A randomized controlled intervention study in human subjects could be set up combining insights from the results of this study and that of a previous study [42] that looked at the potential of adding Mabisi and Munkoyo to the food based recommendations to determine how much of the fermented food should be given to the study subjects for impact. 
1. Marteau P, Gerhardt M, Myara A, Bouvier E, Trivin F, Rambaud J: Metabolism of bile salts by alimentary bacteria during transit in the human small intestine. Microbial ecology in health and disease 1995, 8(4):151-157.

2. Kort R, Sybesma W: Probiotics for every body. Trends in biotechnology 2012, 30(12):613615.

3. WHO F: Evaluation of health and nutritional properties of powder milk and live lactic acid bacteria. Food and Agriculture Organization of the United Nations and World Health Organization Expert Consultation Report 2001:1-34.

4. Macfarlane GT, Cummings JH: Probiotics, infection and immunity. Current opinion in infectious diseases 2002, 15(5):501-506.

5. Vitapole D: Fermented foods and healthy digestive functions: J. Libbey Eurotext; 2001.

6. Reid G, Bruce AW: Could probiotics be an option for treating and preventing urogenital infections? Medscape women's health 2001, 6(5):9-9.

7. Reid G, Beuerman D, Heinemann C, Bruce AW: Probiotic Lactobacillus dose required to restore and maintain a normal vaginal flora. FEMS Immunology \& Medical Microbiology 2001, 32(1):37-41.

8. Kyne L, Kelly C: Recurrent Clostridium difficilediarrhoea. Gut 2001, 49(1):152-153.

9. Guslandi M, Mezzi G, Sorghi M, Testoni PA: Saccharomyces boulardii in maintenance treatment of Crohn's disease. Digestive diseases and sciences 2000, 45(7):1462-1464.

10. Gorbach SL: Probiotics and gastrointestinal health. The American journal of gastroenterology 2000, 95(1):S2-S4.

11. Gionchetti $\mathrm{P}$, Rizzello F, Venturi A, Campieri M: Probiotics in infective diarrhoea and inflammatory bowel diseases. Journal of gastroenterology and hepatology 2000, 15(5):489493.

12. Cunningham-Rundles $S$, Ahrné $S$, Bengmark $S$, Johann-Liang R, Marshall F, Metakis L, Califano C, Dunn A-M, Grassey C, Hinds G: Probiotics and immune response. The American journal of gastroenterology 2000, 95(1):S22-S25.

13. Caplan MS, Jilling T: Neonatal necrotizing enterocolitis: possible role of probiotic supplementation. Journal of pediatric gastroenterology and nutrition 2000, 30:S18-S22.

14. Bengmark S: Colonic food: pre-and probiotics. The American journal of gastroenterology 2000, 95(1):S5-S7.

15. Lee $\mathrm{Y}$, Nomoto K, Salminen S, Gorbach S: Role of probiotics in health and diseases. Handbook of probiotics John Wiley \& Sons, Inc, New York, NY 1999:67-146.

16. Campieri M, Gionchetti P: Probiotics in inflammatory bowel disease: new insight to pathogenesis or a possible therapeutic alternative? In.: Elsevier; 1999.

17. Taranto MP, Medici M, Perdigon G, Ruiz Holgado AP, Valdez GF: Evidence for hypocholesterolemic effect of Lactobacillus reuteri in hypercholesterolemic mice. J Dairy Sci 1998, 81(9):2336-2340.

18. Goldin BR, Gualtieri LJ, Moore RP: The effect of Lactobacillus GG on the initiation and promotion of DMH-induced intestinal tumors in the rat. 1996.

19. Vuyst L, Vandamme EJ: Bacteriocins of lactic acid bacteria: microbiology, genetics and applications: Springer US; 1994.

20. Aso Y, Akazan H: Prophylactic effect of a Lactobacillus casei preparation on the recurrence of superficial bladder cancer. Urologia internationalis 1992, 49(3):125-129.

21. Mallett $A$, Bearne $C$, Rowland $\mathrm{I}$ : The influence of incubation $\mathrm{pH}$ on the activity of rat and human gut flora enzymes. Journal of applied bacteriology 1989, 66(5):433-437.

22. Gorbach S, Chang T-W, Goldin B: Successful treatment of relapsing Clostridium difficile colitis with Lactobacillus GG. The Lancet 1987, 330(8574):1519.

23. Lilly DM, Stillwell RH: Probiotics: growth-promoting factors produced by microorganisms. Science (New York, NY) 1965, 147(3659):747-748. 
24. Gill HS, Guarner F: Probiotics and human health: a clinical perspective. Postgraduate Medical Journal 2004, 80(947):516-526.

25. Fujiwara S, Hashiba H, Hirota T, Forstner JF: Proteinaceous factor (s) in culture supernatant fluids of bifidobacteria which prevents the binding of enterotoxigenic Escherichia coli to gangliotetraosylceramide. Appl Environ Microbiol 1997, 63(2):506-512.

26. Gilliland SE: Health and nutritional benefits from lactic acid bacteria. FEMS Microbiology reviews 1990, 7(1-2):175-188.

27. Fernandes $C$, Shahani $K$, Amer M: Therapeutic role of dietary lactobacilli and lactobacillic fermented dairy products. FEMS Microbiology Reviews 1987, 3(3):343-356.

28. Deeth $\mathrm{H}$, Tamime A: Yogurt: nutritive and therapeutic aspects. Journal of food protection 1981, 44(1):78-86.

29. Chelule P, Mokoena M, Gqaleni N: Advantages of traditional lactic acid bacteria fermentation of food in Africa. Current research, technology and education topics in applied microbiology and microbial biotechnology 2010, 2:1160-1167.

30. Phiri S SS, Van den Heuvel J, Smid EJ, Shindano J, Linnemann AR: Fermented cereal-based Munkoyo beverage: processing practices, microbial diversity and aroma compounds. 2019.

31. Moonga HB, Schoustra SE, Linnemann AR, Kuntashula E, Shindano J, Smid EJ: The art of mabisi production: A traditional fermented milk. PloS one 2019, 14(3):e0213541.

32. Schoustra SE, Kasase C, Toarta C, Kassen R, Poulain AJ: Microbial Community Structure of Three Traditional Zambian Fermented Products: Mabisi, Chibwantu and Munkoyo. PLOS ONE 2013, 8(5):e63948.

33. Tan $\mathrm{H}, \mathrm{O}$ 'Toole PW: Impact of diet on the human intestinal microbiota. Current Opinion in Food Science 2015, 2(0):71-77.

34. Conlon $M$, Bird $A$ : The impact of diet and lifestyle on gut microbiota and human health. Nutrients 2015, 7(1):17-44.

35. Salonen A, De Vos WM: Impact of diet on human intestinal microbiota and health. Annual Review of Food Science and Technology 2014, 5(1):239-262.

36. Gibson GR, Fuller R: Aspects of in vitro and in vivo research approaches directed toward identifying probiotics and prebiotics for human use. The Journal of nutrition 2000, 130(2):391S-395S.

37. Takagi R, Sasaki K, Sasaki D, Fukuda I, Tanaka K, Yoshida K-i, Kondo A, Osawa R: A singlebatch fermentation system to simulate human colonic microbiota for high-throughput evaluation of prebiotics. PLOS One 2016, 11(8):e0160533.

38. Alander M, De Smet I, Nollet L, Verstraete W, von Wright A, Mattila-Sandholm T: The effect of probiotic strains on the microbiota of the Simulator of the Human Intestinal Microbial Ecosystem (SHIME). International journal of food microbiology 1999, 46(1):71-79.

39. Chambers ES, Preston T, Frost G, Morrison DJ: Role of Gut Microbiota-Generated ShortChain Fatty Acids in Metabolic and Cardiovascular Health. Current nutrition reports 2018, 7(4):198-206.

40. Rösch C, Venema K, Gruppen H, Schols HA: Characterisation and in vitro fermentation of resistant maltodextrins using human faecal inoculum and analysis of bacterial enzymes present. Bioactive carbohydrates and dietary fibre 2015, 2015 v.6 no.1(no. 1):pp. 8-53.

41. De Baere S, Eeckhaut V, Steppe M, De Maesschalck C, De Backer P, Van Immerseel F, Croubels S: Development of a HPLC-UV method for the quantitative determination of four short-chain fatty acids and lactic acid produced by intestinal bacteria during in vitro fermentation. Journal of pharmaceutical and biomedical analysis 2013, 80:107-115.

42. Justin Chileshe EFT, Sijmen E Schoustra, Karin J Borgonjen-van den Berg, Ray Handema, Bas J Zwaan and Inge D Brouwer: Potential contribution of cereal and milk based fermented foods to dietary nutrient intake of 1-5 years old children in Central province in Zambia. 2019. 
Supplementary Table 7: Repeated measures one-way MANOVA results after applying Tukey HSD post hoc test for multiple comparisons between replicates ( $I$ and J)for each treatment. The table indicates the differences between replicates for the different treatments and the mean differences are positive when the first replicate (I) has a higher value for the SCFAs than the second replicate (J) and this is tested for significance indicated by Sig. in the table and the $95 \% \mathrm{Cl}$ is provided. The dependent variable is the combined SCFAs.

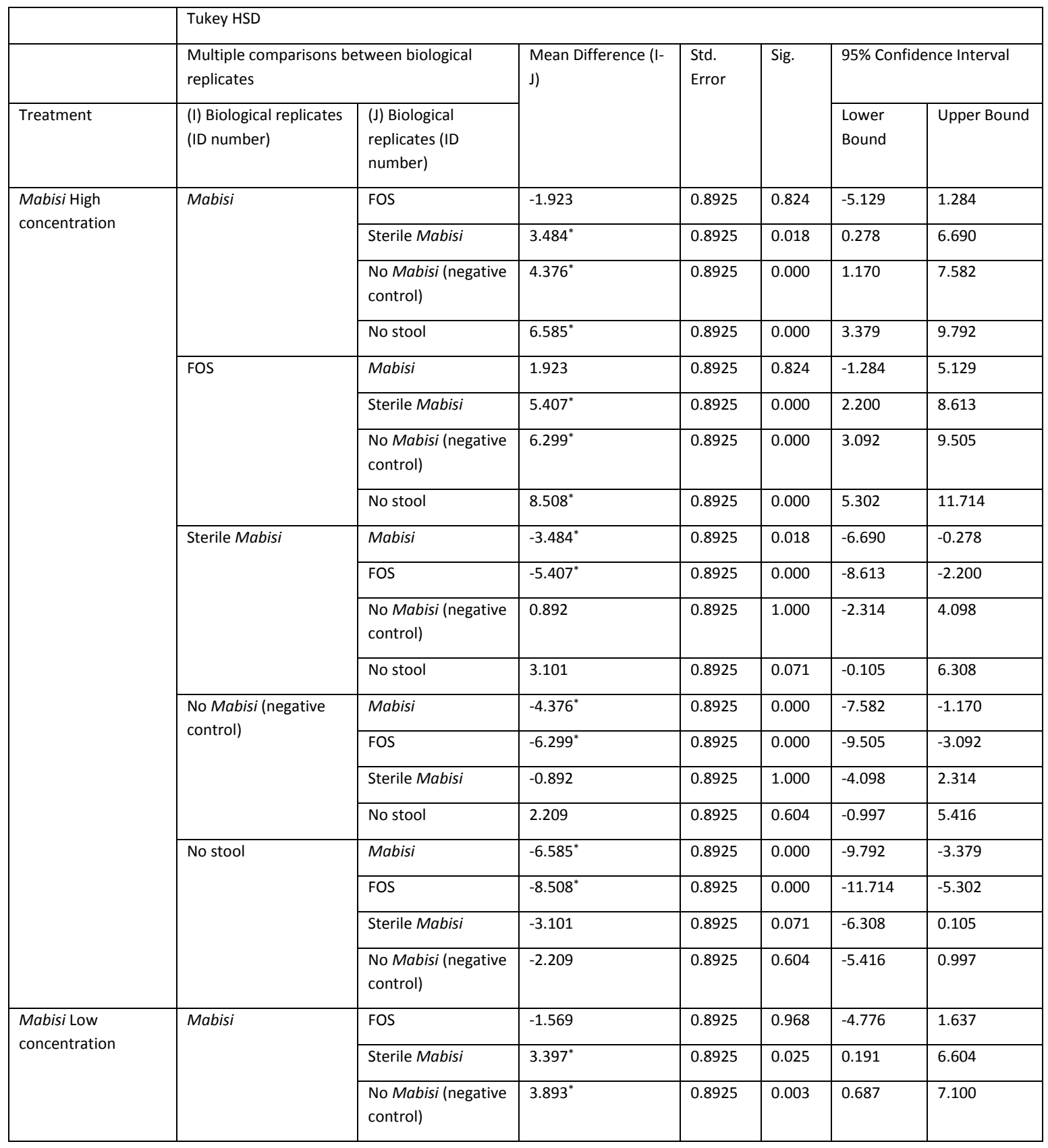




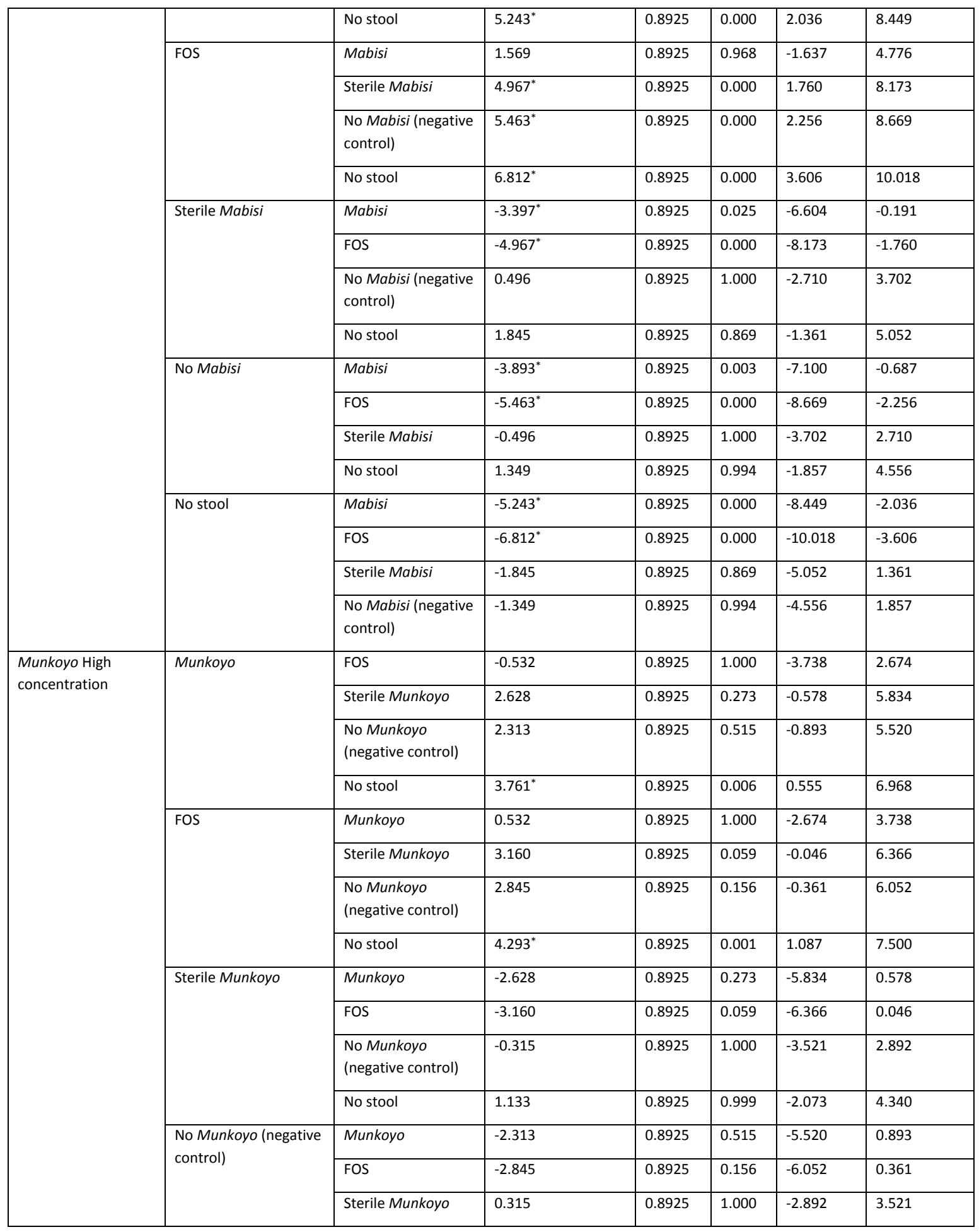




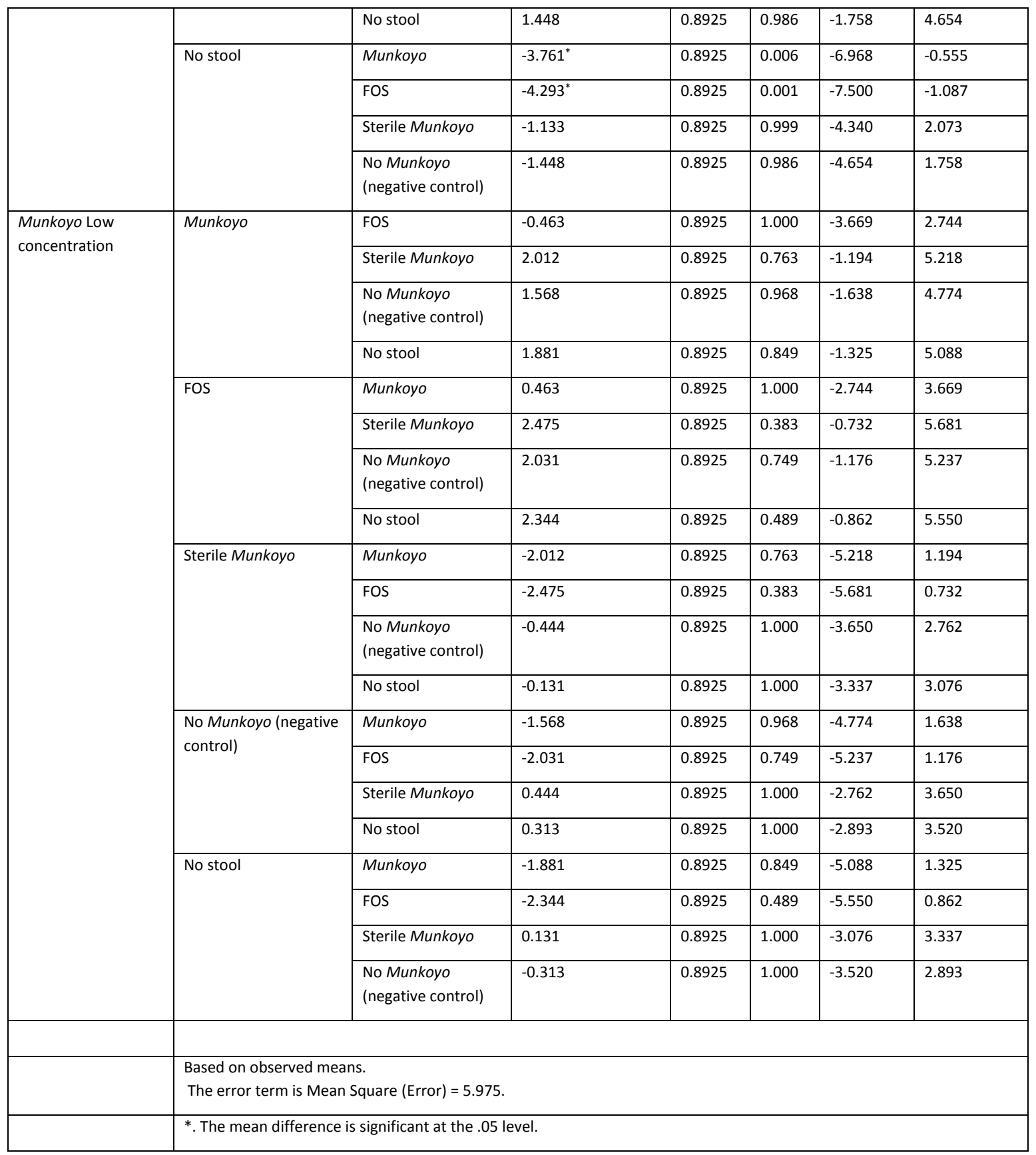



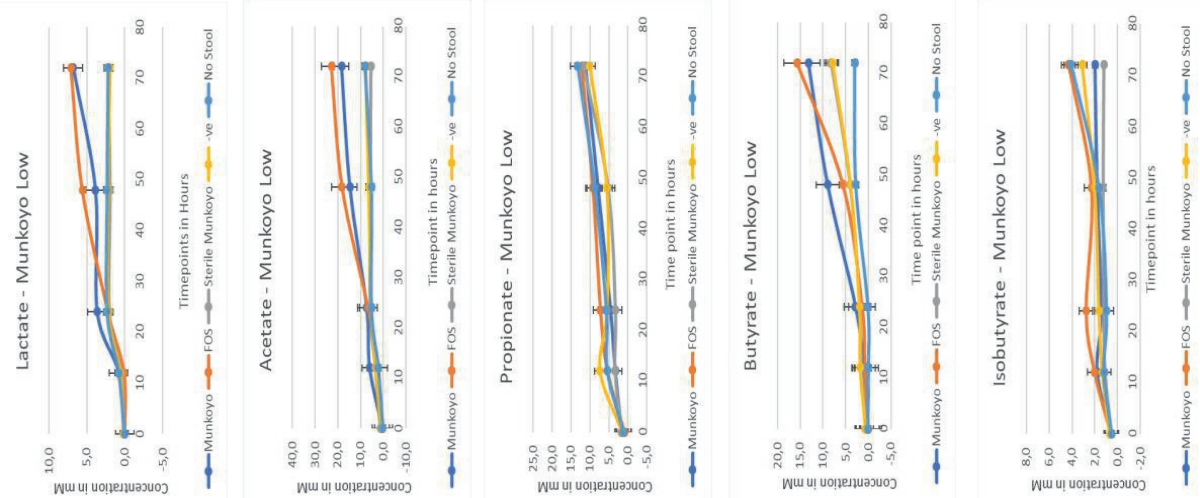

3
0
0
1
5
2
0
0
0
0
0
0
2
5
5
3
3
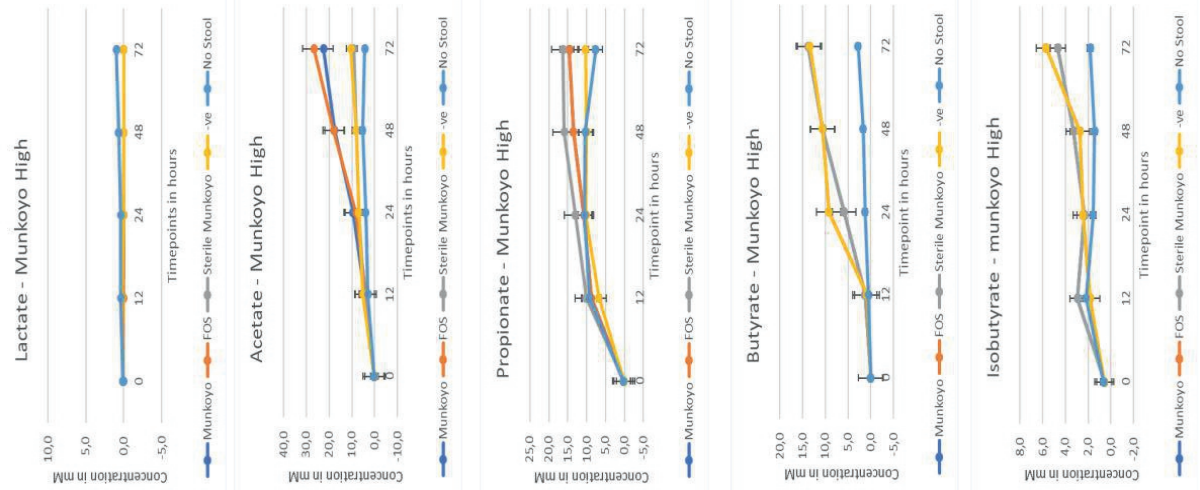

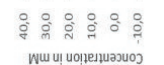

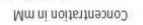

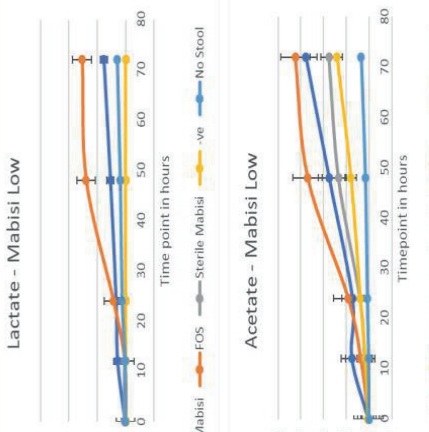

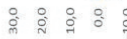

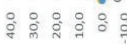

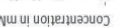
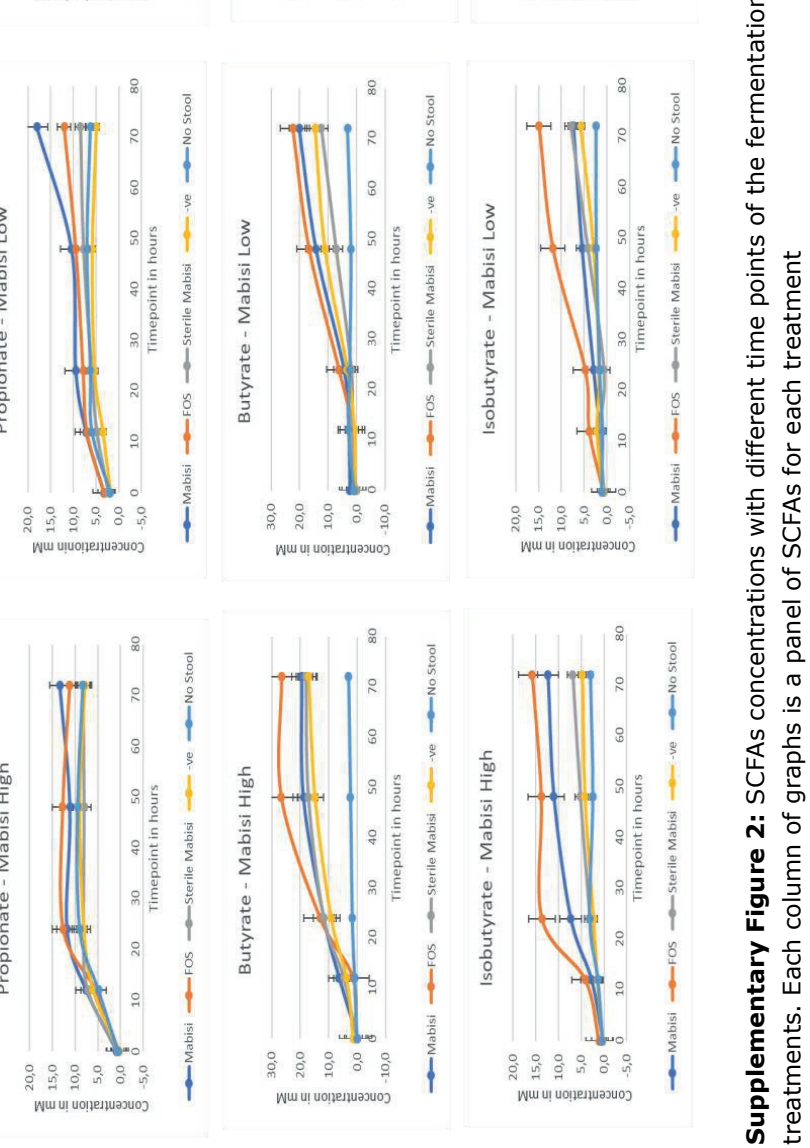
Chapter 5 
Effects of traditional fermented foods consumption on gut microbial composition in 6-24 months old children in rural Zambia

Justin Chileshe ${ }^{1,3}$, Pamela A. Marinda ${ }^{2}$, Bubala T. Hamaimbo ${ }^{2}$, Jay Sikalima ${ }^{1}$, Ray Handema ${ }^{1}$, Bas J. Zwaan $^{3}$, Elise F. Talsma ${ }^{4}$ and Sijmen E. Schoustra ${ }^{2,3}$

${ }^{1}$ Tropical Diseases Research Centre, Biomedical Sciences Department, Ndola, Zambia

${ }^{2}$ The University of Zambia, School of Agricultural Sciences, Department of Food Science and Nutrition, Lusaka, Zambia

${ }^{3}$ Wageningen University, Laboratory of Genetics, Wageningen, the Netherlands

${ }^{4}$ Wageningen University and Research, Division of Human Nutrition and Health, the Netherlands 


\section{Abstract}

The human microbiota consists of a complex community of microorganisms that play an important role in immunity, nutrition and metabolism, and structure and function of the gastro intestinal tract. Diet is one of the important factors that affect the gut microbiota, with fermented foods generally known to be beneficial in modulating the gut microbiota. In Zambia many traditional fermented foods exists that are important in the diets of various groups. The aim of this study was to determine the impact of consuming traditional fermented foods Mabisi and Munkoyo on the gut microbiota their metabolism in children. Data on food consumption, morbidity and socio-demographic together with stool samples were collected from children aged 6-24 months residing in Namwala and Mkushi in Zambia. The stool samples were analysed for the composition of gut microbiota and concentrations of short chain fatty acids (SCFAs) as a proxy for microbial metabolism. This data was then linked to data on intake of traditional fermented foods and data analysis was performed using multivariate analysis of variance (MANOVA). Gut microbiota of children who are fermented beverages consumers was associated with higher relative abundance of Bacteroides and Lactobacillus than the non-consumers. Higher levels of these bacterial groups have been associated with benefits to the host. There was no difference for the SCFA concentrations between the fermented foods consumers and non-consumers, which may be because other parts of the diets that we did not control for also contribute to SCFA production. The results imply that consumption of the two traditional fermented foods used in this study promotes a healthier gut microbiota composition at the level of gut microbiota composition in the children in Zambia. Our results warrant future more formal tests such as controlled human feeding trials to further validate our findings.

\section{Introduction}

The human microbiota consists of a complex community of microorganisms with the majority residing in the large intestine. A healthy gut microbiota composition is described by its richness and diversity and it is shaped in early life, but this optimal healthy gut microbiota composition differs between individuals [1]. The gut microbes play a role in immunity, nutrition and metabolism, and structure and function of the gastro-intestinal (GI) tract [2-4]. The microorganisms thought responsible for the microbiomeimproving effect of diets can also be administered as a probiotics which have been defined by the World Health Organization (WHO) as "live microorganisms which when administered in adequate amounts confer a health benefit on the host" [5]. Most of these probiotic bacteria belong to the class of lactic acid bacteria. Diets or intake of certain foods can affect gut microbiota composition. This was shown by 
the consumption of certain dietary fibers and products containing bacteria of which probiotic properties have been formally demonstrated, for instance for undernourished children [6].

Traditional or unformalized fermented foods contain a wide variety and diverse community of lactic acid and acetic acid bacteria [7]. The technique of fermentation relies on the activity of microbes and has been used globally to improve nutritional, safety, and organoleptic properties of food using different raw materials [8-10] [11]. While formalized fermented foods are commonly produced using starter cultures that contain only a few types of bacteria, most traditional unformalized fermentation processes rely on the activity of mixture of various lactic acid bacteria, such as Lactobacillus, Lactococcus, and Leuconostoc $[7,12]$. These bacteria are of the same bacterial genera as bacteria that have been classified as probiotic bacteria and for which probiotic properties have formally been demonstrated [13]. These traditional fermented foods may thus naturally contain beneficial bacteria and their metabolic products (such as short chain fatty acids, SCFAs). Thus, these products may confer nutritional and health benefits to the consumer by shifting the microbial gut community towards a higher abundance of species that are considered beneficial for health, such as lactic acid bacteria [3, 14]. Microbial fermentation of dietary fibre in human large intestines leads to production of SCFAs which play an important role in modulating the health of the host through a range of tissue-specific mechanisms, among others related to gut barrier function, glucose homeostasis, immunomodulation, appetite regulation and obesity among other functions [15].

There is limited knowledge on the impact of traditional fermented foods on gut microbial content, nutritional status, and SCFAs production in humans. Most studies have been conducted on commercially prepared fermented beverages that are enriched with specific bacterial strains and their probiotic effects with impact on gut microbiota and health outcomes have formally been demonstrated [16-35]. Studies such as randomized control trials to assess the probiotic effects of traditional fermented foods on gut microbiota modulation and health outcomes are challenging to conduct. For instance, next to ethical issues related to involving human subjects, what should be the amount and duration of consumption for the effects to be visible, and how can the same quality of products be guaranteed for the period of the study? As a result, not much is known about the probiotic potential of traditional fermented foods [7] and no formal recommendations have been made for sufficient intake levels for impact on the gut microbiota and subsequently on the health of the individual consumer.

In this study we specifically evaluate the effect of two traditional fermented foods in Zambia to promote a healthy gut microbiota in children between 6 and 24 months of age in two rural areas. In Zambia traditional non-alcoholic fermented beverages are part of the daily diet and consumed by all age groups with the most common ones being Mabisi (based on milk) and Munkoyo/Chibwantu (based on cereal). 
These two fermented beverages are made through transfer of a small fraction of the previous product into fresh raw material (back-slopping), in this way transferring the microbial communities underlying the fermentation from batch to batch, or through repeatable spontaneous fermentation. Previous studies have found that the fermenting microbial communities of Mabisi and Munkoyo/Chibwantu were dominated by around six to ten species of lactic acid and acetic acid bacteria [36, 37]. The predominance of strains of lactic acid bacteria suggests that probiotic properties may be attributed to these products.

Here, we correlated consumption of these two fermented foods to gut microbiota composition of its consumers in two locations in rural Zambia with different intake levels of traditional fermented foods. This study complements a previous study in which we found that nutritional status and consumption of traditional fermented foods was significantly higher in Namwala than in Mkushi and that consumption of Mabisi was negatively associated with diarrhoea and stunting while Munkoyo/Chibwantu consumption was negatively associated with vomiting [38]. We have collected stool samples in an earlier study [38] and now analysed these stool samples for their composition of gut microbiota and concentrations of SCFAs. We correlated this to data on intake of traditional fermented foods to understand whether consumption of fermented foods leads to healthier gut microbiota composition and higher levels of SCFAs.

\section{Methods}

\section{Study design}

\section{Study site, target population, and dietary intake data collection}

The study was conducted in Mkushi district (Central Province, GPS coordinates: $13.6212^{\circ} \mathrm{S}, 29.3889^{\circ} \mathrm{E}$ ) and Namwala district (southern province, GPS coordinates: $15.7525^{\circ} \mathrm{S}, 26.4371^{\circ} \mathrm{E}$ ) of Zambia in children aged 6-24 months as described by Hamaimbo et al. [38]. These districts were selected to represent areas with high (Namwala) and one with low (Mkushi) consumption of Mabisi (the fermented milk). Munkoyo/Chibwantu consumption rates are roughly equal in both areas. In the two areas communities were divided into clusters (one with children consuming fermented foods regularly and the other cluster of children who do not usually consume fermented foods). Children were included if they were aged between 6-24 months, their parents/guardians consented to participating and children did not have chronic health conditions as reported by the parents/guardians. The food intake data was collected using 24-hour recall and food frequency questionnaire and anthropometric measurements were collected for calculating Z-scores of height-for-age, weight-for-height, and weight-for-age using WHO AnthroPlus version 1.0.4. Socio-demographic and morbidity data were also collected [38]. 
Stool samples were collected in sterile stool containers from the study participants and were stored in the freezer at $-20^{\circ} \mathrm{C}$. The samples were transported to the TDRC laboratory in a cool box with icepacks and stored at $-80^{\circ} \mathrm{C}$ until analysis of their microbial composition and concentrations of short chain fatty acids.

Ethical clearance was obtained from the Tropical Diseases Research Centre Ethics Review Committee, Zambia (STC/2017/16) and the University of Zambia Biomedical Research Ethics Committee, Zambia (Ref. No. 002-04-18) and further permission was obtained from the National Health Research Authority of the Ministry of Health.

\section{Laboratory methods}

Stool sample DNA: Whole bacterial genomic DNA from each stool sample was prepared by taking an amount of $150 \mathrm{mg}$ of stool into a sterile bead-beating tube containing $300 \mathrm{mg}$ of glass beads (diameter $0.1 \mathrm{~mm}$ ) using the Zymo Research Quick DNA faecal/soil microbe microprep kit (Cat \# 6010 and 6012) for DNA isolation in stool samples after bead-beating in a Sunon mini bead-beater according to manufacturer's instructions.

Determination of bacterial composition (major groups of bacteria) in stool samples. A method adapted from Takagi et al. was used for this analysis [39]. This method is based on real-time quantitative polymerase chain reaction ( $\mathrm{qPCR}$ ) aimed at determining the relative abundance of several major classes of bacterial genera within the gut microbiota. We used an Applied Biosystems AB7500 Real Time thermocycler with the primer sets and reaction conditions described in Table 1 used for measuring the partial 16S rRNA gene copy number of All Eubacteria, Bacteroides, Lactobacillus, Bifidobacteria, and Enterococcus. All quantitative measurements by real-time PCR were done in duplicate. The real-time PCR amplification program for Eubacteria was as follows: $95^{\circ} \mathrm{C}$ for $3 \mathrm{~min}$, followed by 38 cycles of $95^{\circ} \mathrm{C}$ for $30 \mathrm{sec}, 54^{\circ} \mathrm{C}$ for $30 \mathrm{sec}$ and $72^{\circ} \mathrm{C}$ for $50 \mathrm{sec}$. The real-time PCR amplification program for the genus Bifidobacterium was as follows: $94^{\circ} \mathrm{C}$ for $5 \mathrm{~min}$, followed by 40 cycles of $94^{\circ} \mathrm{C}$ for $20 \mathrm{sec}, 55^{\circ} \mathrm{C}$ for $20 \mathrm{sec}$ and $72^{\circ} \mathrm{C}$ for $50 \mathrm{sec}$. To check the specificity of the amplifications, a melting curve was obtained by performing the following cycle: a denaturation step at $95^{\circ} \mathrm{C}$ for $15 \mathrm{sec}$, a $1^{\circ} \mathrm{C}$ increase in temperature every $20 \mathrm{sec}$ starting at $60^{\circ} \mathrm{C}$ and ending at $95^{\circ} \mathrm{C}$, and a final step at $95^{\circ} \mathrm{C}$ for $15 \mathrm{sec}$. Standard curves for absolute quantification of the samples were prepared using the standards from DSM (Lactobacillus plantarum DSM20205, Bacteroides ovatus DSM1896, Enterococcus casseliflavus DSM4841 and Bifidobacterium catenulatum DSM16992) and for all eubacteria, Escherichia coli ATCC25922 standard strain was used targeting the PCR fragments of the $16 \mathrm{~S}$ rRNA genes. The correlation coefficients for all the standard curves exceeded 0.99 . For each assay, $2 \mu \mathrm{L}$ of DNA solution was added to $18 \mu \mathrm{L}$ of a PCR 
mixture containing $10 \mu \mathrm{L}$ of THUNDERBIRD' ${ }^{\text {TM }}$ SYBR1 qPCR Mix (Applied Biosystems), 7.2 $\mu \mathrm{L}$ of distilled water, and $200 \mathrm{nM}$ of each primer.

Determination of the composition of short-chain fatty acids (SCFAs). Concentrations of acetate, propionate, butyrate, lactate, isobutyrate, and succinate were measured using a Shimadzu LC-2010CHT high performance liquid chromatograph (HPLC) as described elsewhere [40]. About 300mg of sample was weighed into a glass tube with screw cap and $3 \mathrm{ml}$ of normal saline was added to homogenize by vortexing. The SCFAs were extracted by adding $5 \mathrm{ml}$ of diethyl ether to the solution, incubated at room temperature while shaking for 30 minutes and centrifuged. The organic supernatant was transferred to another clean tube and the extraction process was repeated and the organic layers were combined and evaporated under a gentle stream of nitrogen gas. The residue was dissolved in $50 \mu$ l of the mobile phase solution and $20 \mu \mathrm{l}$ was injected into the HPLC system. The HPLC was equipped with a Hypersil Gold aQ (150mm x 4.6mm, dp: $3 \mu \mathrm{m}$ ) column (Thermal Fischer Scientific, cat \#: $25303-154630$ ) operated at $65^{\circ} \mathrm{C}$ and a photo diode array detector (Shimadzu) using $5 \mathrm{mM} \mathrm{H}_{2} \mathrm{SO}_{4}$ as the mobile phase at a flow rate of 0.6 $\mathrm{mL} / \mathrm{min}$. Peak areas for each SCFA were integrated and used for calculating the concentration from the calibration curves.

Data analysis. For microbial data, the output dataset including $\mathrm{Ct}$ number, bacteria name, sample ID, concentration, and replicate was opened in Microsoft ${ }^{\circledR}$ Excel directly from the exported $\mathrm{Ct}$ file from the AB 7500 qPCR machine. Copy number per microliter of sample for each bacterium was calculated and data was then exported into SPSS (version 25) for statistical analysis after data from the HPLC analysis of the Short-Chain Fatty acids was also added matching the ID and replicate number. In the data, the study participants were allocated to four groups according to the type of fermented food they consumed or not as: non-consumers, those that consumed only Mabisi, those that consumed only Munkoyo/Chibwantu, and those that consumed both.

A non-parametric ANOVA on bacterial count data was performed to test for effect of fermented foods consumption on different bacterial types and SCFAs for the four consumer groups. Further, we performed multivariate General Linear Regression (GLS) and used Pillai's Trace as a test for significance with combined dependent variable firstly comprising of Bacteroides, Lactobacillus, Bifidobacteria and Enterococcus abundance and secondly comprising of SCFAs. Different independent variables of sociodemographic characteristics, morbidity, intake of different food including Mabisi and Munkoyo/Chibwantu were tested in the model as shown in supplementary Table 3. The model used was: $Y_{i j}=\beta_{0 j}+\beta_{1 j} X_{i 1}+\ldots+\beta_{p j} X_{i p}+\varepsilon_{i j}$, where $Y_{i}$ is the $i^{\text {th }}$ observation of the dependent variable, $X_{i j}$ is $i^{\text {th }}$ observation of the $j^{\text {th }}$ independent variable, $j=1,2, \ldots, p$. Values $\beta_{j}$ represent parameters to be estimated, 
and $\varepsilon_{i}$ is the $i^{\text {th }}$ independent error term; for all observations indexed as $i=1, \ldots, n$ and for all dependent variables indexed as $j=1, \ldots, \mathrm{m}[41]$.

After excluding a number of socio-demographic, morbidity and food intake variables, another MANOVA was performed with the independent variables that were significant in the first MANOVA. Tukey HSD as post hoc test was performed to evaluate whether there were differences or not between consumers and non-consumers of fermented foods for the different types of bacteria and the SCFAs.

\section{Results}

In this study we analyzed stool samples of children 6-24 months old in Namwala and Mkushi. A total of 126 stool samples were collected, 58 in Namwala district and 68 in Mkushi district. The response rate for stool sample collection was $90 \%$. The socio-demographic characteristics and morbidity of the study participants have been described elsewhere [38] and are shown in supplementary Tables 4 and 5. Among the respondents, we defined four consumer groups based on their consumption patterns of the fermented foods (non-consumers, only consuming Mabisi, only consuming Munkoyo/Chibwantu and consuming both). Figure 1 (panels $A$ and $B$ ) shows the relative abundance of the four focal bacterial groups (as fraction of all Eubacteriał per sampling location (Namwala and Mkushi) differentiating between the four different consumer groups.

We found an effect of combined fermented foods consumption on the dependent variable (combined bacteria: Bacteroides, Lactobacillus, Bifidobacteria and Enterococcus). This was shown by two statistical analyses. Firstly, a Mann-Whitney-Wilcoxon test to determine the differences for the various classes of bacteria and SCFAs between consumers and non-consumers of fermented foods for each area (Namwala and Mkushi). Table 2 shows that overall (combining all consumers of both areas) the relative abundance of Bacteroides and Lactobacillus are significantly higher for consumers of fermented foods, while levels of Bifidobacteria and Enterococcus are not statistically significantly different. When analysed per area, we found similar results, however these were no longer statistically significant due to low number of samples in each consumer group limiting statistical power. When comparing consumers of one type of product (either Mabisi or Munkoyo/Chibwantu) and non-consumers, we found the same general patterns.

Secondly, we performed a multivariate general linear regression and Tukey's HSD Post hoc tests to test the effects of different independent variables of socio-demographic characteristics, morbidity, and (other) food intake. The main results can be summarized as follows: We confirmed that consumption of both fermented foods for the combined sites was strongly associated specifically with an increase in abundance of Bacteroides $(F(3)=2.928, P=0.038)$ and Lactobacillus $(F(3)=4.259, P=0.007)$. Then, we assessed whether the observed increase in relative abundance levels of bacteria could be associated 
with other factors while still including consumption of fermented foods. Based on Pillai's Trace multivariate test consumption of fermented foods $\left(F=2.001, P=0.009, \eta^{2}=0.185\right)$ was significantly associated with the increase in relative abundance of the bacteria but also with the following independent variables: child's age $\left(F=2.628, P=0.048, \eta^{2}=0.204\right)$ and having domestic animals $(F=$ 3.014, $\left.P=0.029, \eta^{2}=0.227\right)$; stunting (height-for-age, Z-score, $\left.H A Z\right)\left(F=3.618, P=0.013, \eta^{2}=0.261\right.$ ); malaria $\left(F=3.736, P=0.011, \eta^{2}=0.267\right)$; consumption of spinach $\left(F=2.819, P=0.037, \eta^{2}=0.216\right)$, mangoes $\left(F=4.176, P=0.006, \eta^{2}=0.289\right)$, other fruits and vegetables (food group $\left.4-F G 4\right)(F=4.323, P$ $\left.=0.005, \eta^{2}=0.297\right)$, fowl eggs $\left(F=3.245, P=0.021, \eta^{2}=0.240\right)$, beef $\left(F=4.004, P=0.008, \eta^{2}=0.281\right)$, other foods $\left(F=3.477, P=0.016, \eta^{2}=0.253\right)$ and oils $\left(F=3.088, P=0.026, \eta^{2}=0.232\right)$.

The results show similar SCFAs concentrations for the four consumer groups except for lactic acid, whose concentrations were significantly higher for non-consumers than consumers of any of the fermented foods (Figure 1, panels C and D). The total concentrations of SCFAs in Mkushi were highest for nonconsumers except for lactic acid, but this was not confirmed for the Namwala site. When the multivariate general linear regression analysis was conducted using the SCFAs (lactic acid, acetic acid, propionic acid, butyric acid and isobutyric acid) as combined dependent variables in the same way as for the bacteria, consumption of fermented foods did not affect the SCFAs concentrations in the studied population.

\section{Discussion}

In this study we assessed the effect of traditional fermented foods consumption on the gut bacterial composition and the concentration of short chain fatty acids (SCFAs). We linked consumption patterns of traditional fermented foods of children aged between 6-24 months to the results of stool samples collected from the same individuals. Relative abundance of Bacteroides, Lactobacillus, Bifidobacteria, and Enterococcus were the indicators of beneficial classes of bacteria in the gut microbiota. Overall, we found that the abundance of Bacteroides and Lactobacillus among the gut microbiota was higher in consumers of fermented foods than non-consumers. This result is in line with our expectation that consumption of traditional fermented foods that contain live bacteria enhances the level of the same classes of bacteria as those found in the gut microbiome. When analysing effects of consumption per area or per product type, our results are similar to the overall result, yet no longer statistically significant due to the low number of respondents per class.

We further asked if consumption of fermented foods is associated with increased abundance of bacterial types Bacteroides and Enterococcus even when correcting for other socio-demographic, morbidity and food consumption factors that also affect the gut microbiome. We found that bacterial abundance is 
associated with child's age, reduced levels of stunting, reduced levels of malaria, keeping domestic animals, consuming foods such as spinach, mango, fruits and vegetables, fowl eggs, beef, oils and fermented foods. These findings confirm that children with higher consumption of fermented beverages have a higher abundance of bacteria and also a better nutritional status as measured in levels of stunting, in line with well-established information on the ability of diet especially fermented foods and beverages to modulate the gut microbiota to a composition that can promote health $[42,43]$. Consumers typically consume around $183 \mathrm{~g}$ per day [44]. Our results suggest that the inclusion of one such serving can already have a positive effect on gut microbiota composition. The results (shown in Table 2 and supplementary Table 3) are also consistent with the idea that increase in abundance of certain commensal bacterial species such as lactic acid bacteria can be one of the key mechanisms underlying the beneficial effects of fermented food on gut function, immune modulation and metabolism [45].

Our measurements of concentrations of SCFAs overall did not show an effect of consumption of fermented foods. These SCFAs are primarily produced through microbial fermentation of dietary fibre in human large intestines and play an important role in modulating host health [15]. Since SCFAs are products of fermentation by gut bacteria, we expected that their concentrations would be higher following the pattern of increased gut bacteria in fermented foods consumers than non-consumers. It could be that the children had the same amounts of fermentable fibers from other parts of the diet that we did not control for available in their gut whether they consumed the fermented foods or not and as a result produced the same amounts of SCFAs. Our results are in contrast to other findings which confirmed that consumption of probiotics positively affects gut microbiota and consequently increases production of SCFAs $[13,15,46]$. The lack of effect of consumption of fermented foods could be attributed to a number of factors. One of the factors could have been due to the time lapse between collection and freezing the samples as some sites were far and took more hours than the nearby sites. Also the time between collection and analysis of the stool samples could have affected the results of our study as the samples were only analysed after three weeks, as other studies have found mostly a decrease in SCFAs concentrations in relation to storage time [47]. More generally, the idea that SCFA are a useful proxy for health promoting bacterial metabolism by the gut microbiota is being debated and conflicting information exist. For instance in a recent study showing that higher levels of SCFA are not associated with reduced risk of cancer [48]. On the other hand, a recent review clearly highlights the beneficial effects of SCFAs on preventing inflammatory disorders [49].

Our study has several strengths and weaknesses. A weakness is that information on consumption of fermented foods was collected by questionnaire and this could have led to over or under-estimation. Our survey approach to look for a relationship between consumption of fermented foods and gut 
microbiota composition should be seen as a first step to justify a more formal and elaborate test would be interesting. Such a test would include conducting a randomized clinical nutrition intervention, which would be the best method [50] to assess efficacy of fermented products on gut microbiota composition, nutritional status, and health and which possibly would give a more unequivocal signal. Ideally one would like to test whether consumption of traditional fermented foods not only contributes to a healthier gut microbiota composition, but also to a better nutritional status and health. Combining our current findings with those from our previous study on the nutritional status of the same respondents [38], we are able to infer some correlations, for instance a positive correlation between consumption of fermented foods and lower levels of stunting. While this result from the regression analysis showed an interesting trend in support of the notion that consumption of traditional fermented foods promotes nutritional status, formal tests, and follow-ups such as controlled feeding trials and randomised clinical trials (RCT) are required to show potential causation.

The data were collected using a one-time point cross-sectional method and that we could not effectively capture the health-seeking behaviour of the study participants which could have affected the results of the study. Especially, while we did exclude participants with a chronic illness, we did not collect information on whether the study participants had taken any antibiotics preceding the study or not which could have helped to interpret the results for bacterial counts. We have however elicited important data pointing to the potential of traditional fermented beverages Mabisi and Munkoyo/Chibwantu consumption to impact on the composition of the gut microbiota which generally is considered to promote nutritional status and general health.

Nevertheless, it is striking that we found an increase in the relative abundance of relevant bacterial types generally known to promote a healthy gut microbiota, despite the fact that we used a survey approach and not a RCT but only controlled the diet and socio-economic status of respondents in the analysis. A strength of our study is that we looked at the most vulnerable study population (children 6-24 months of age) that has not been considered before in the studies involving traditional fermented foods. Data showed that children stunted by the age of two years do not meet their full potential of physical and mental development later on in life [51] and therefore having interventions at this stage of life would result in beneficial impact on the children's health that lasts throughout life. To date a number of studies have tested the impact of fermented foods mostly produced through the use of starter cultures of specific bacteria [46]. Thus, finally, another strength is that we looked at traditional fermented beverages (based on milk and cereal) that contain around six to ten species of lactic acid and acetic acid bacteria. 
Gut microbiota of children who are fermented beverages consumers was associated with higher relative abundance of Bacteroides and Lactobacillus than the non-consumers. We did not however find any impact of fermented foods consumption on SCFAs in the study population. Namwala being a high area of Mabisi consumption also was found to have better nutrition status [38] and consequently had higher copy numbers of the different types of bacteria tested but these were not statistically significant. However, to support and confirm our promising results, it is recommended that randomised controlled human feeding trials be conducted in human subjects to determine the impact of traditional fermented beverages consumption on gut bacteria modulation as well as the possible interaction between human gut system and fermented beverages. 
1. Rinninella $E$, Raoul $P$, Cintoni $M$, Franceschi $F$, Miggiano GAD, Gasbarrini $A$, Mele MC: What is the Healthy Gut Microbiota Composition? A Changing Ecosystem across Age, Environment, Diet, and Diseases. Microorganisms 2019, 7(1):14.

2. Lazar V, Ditu L-M, Pircalabioru GG, Gheorghe I, Curutiu C, Holban AM, Picu A, Petcu L, Chifiriuc MC: Aspects of Gut Microbiota and Immune System Interactions in Infectious Diseases, Immunopathology, and Cancer. Frontiers in Immunology 2018, 9(1830).

3. Thursby E, Juge N: Introduction to the human gut microbiota. The Biochemical journal 2017, 474(11):1823-1836.

4. Ramakrishna BS: Role of the gut microbiota in human nutrition and metabolism. $J$ Gastroenterol Hepatol 2013, 28 Suppl 4:9-17.

5. WHO F: Evaluation of health and nutritional properties of powder milk and live lactic acid bacteria. Food and Agriculture Organization of the United Nations and World Health Organization Expert Consultation Report 2001:1-34.

6. Kort R, Sybesma W: Probiotics for every body. Trends in Biotechnology 2012, 30(12):613-615.

7. Franz CMAP, Huch M, Mathara JM, Abriouel H, Benomar N, Reid G, Galvez A, Holzapfel WH: African fermented foods and probiotics. International Journal of Food Microbiology 2014, 190:84-96.

8. Nout MR, Sarkar PK, Beuchat LR: Indigenous fermented foods. In: Food Microbiology: Fundamentals and Frontiers, Third Edition. American Society of Microbiology; 2007: 817-835.

9. Mokoena MP, Chelule PK, Gqaleni N: Reduction of fumonisin B1 and zearalenone by lactic acid bacteria in fermented maize meal. Journal of food protection 2005, 68(10):2095-2099.

10. Egounlety M, Aworh OC, Akingbala JO, Houben JH, Nago MC: Nutritional and sensory evaluation of tempe-fortified maize-based weaning foods. Int J Food Sci Nutr 2002, 53(1):1527.

11. Galati A, Oguntoyinbo FA, Moschetti G, Crescimanno M, Settanni L: The cereal market and the role of fermentation in cereal-based food production in Africa. Food reviews international 2014, 30(4):317-337.

12. Heller KJ: Probiotic bacteria in fermented foods: product characteristics and starter organisms. Am J Clin Nutr 2001, 73(2 Suppl):374s-379s.

13. Fijan S: Microorganisms with claimed probiotic properties: an overview of recent literature. Int J Environ Res Public Health 2014, 11(5):4745-4767.

14. Bell V, Ferrão J, Pimentel L, Pintado M, Fernandes T: One Health, Fermented Foods, and Gut Microbiota. Foods (Basel, Switzerland) 2018, 7(12):195.

15. Chambers ES, Preston T, Frost G, Morrison DJ: Role of Gut Microbiota-Generated Short-Chain Fatty Acids in Metabolic and Cardiovascular Health. Current nutrition reports 2018, 7(4):198206.

16. Macfarlane GT, Cummings JH: Probiotics, infection and immunity. Current opinion in infectious diseases 2002, 15(5):501-506.

17. Vitapole D: Fermented foods and healthy digestive functions: J. Libbey Eurotext; 2001.

18. Reid G, Bruce AW: Could probiotics be an option for treating and preventing urogenital infections? Medscape women's health 2001, 6(5):9-9.

19. Reid G, Beuerman D, Heinemann C, Bruce AW: Probiotic Lactobacillus dose required to restore and maintain a normal vaginal flora. FEMS Immunology \& Medical Microbiology 2001, 32(1):37-41.

20. Kyne L, Kelly C: Recurrent Clostridium difficilediarrhoea. Gut 2001, 49(1):152-153.

21. Guslandi M, Mezzi G, Sorghi M, Testoni PA: Saccharomyces boulardii in maintenance treatment of Crohn's disease. Digestive diseases and sciences 2000, 45(7):1462-1464.

22. Gorbach SL: Probiotics and gastrointestinal health. The American journal of gastroenterology 2000, 95(1):S2-S4. 
23. Gionchetti P, Rizzello F, Venturi A, Campieri M: Probiotics in infective diarrhoea and inflammatory bowel diseases. Journal of gastroenterology and hepatology 2000, 15(5):489493.

24. Cunningham-Rundles $S$, Ahrné $S$, Bengmark S, Johann-Liang R, Marshall F, Metakis L, Califano C, Dunn A-M, Grassey C, Hinds G: Probiotics and immune response. The American journal of gastroenterology 2000, 95(1):S22-S25.

25. Caplan MS, Jilling T: Neonatal necrotizing enterocolitis: possible role of probiotic supplementation. Journal of pediatric gastroenterology and nutrition 2000, 30:S18-S22.

26. Bengmark S: Colonic food: pre-and probiotics. The American journal of gastroenterology 2000, 95(1):S5-S7.

27. Lee $Y$, Nomoto K, Salminen S, Gorbach S: Role of probiotics in health and diseases. Handbook of probiotics John Wiley \& Sons, Inc, New York, NY 1999:67-146.

28. Campieri M, Gionchetti P: Probiotics in inflammatory bowel disease: new insight to pathogenesis or a possible therapeutic alternative? In.: Elsevier; 1999.

29. Taranto MP, Medici M, Perdigon G, Ruiz Holgado AP, Valdez GF: Evidence for hypocholesterolemic effect of Lactobacillus reuteri in hypercholesterolemic mice. J Dairy Sci 1998, 81(9):2336-2340.

30. Goldin BR, Gualtieri LJ, Moore RP: The effect of Lactobacillus GG on the initiation and promotion of DMH-induced intestinal tumors in the rat. 1996.

31. Vuyst L, Vandamme EJ: Bacteriocins of lactic acid bacteria: microbiology, genetics and applications: Springer US; 1994.

32. Aso Y, Akazan H: Prophylactic effect of a Lactobacillus casei preparation on the recurrence of superficial bladder cancer. Urologia internationalis 1992, 49(3):125-129.

33. Mallett $A$, Bearne $C$, Rowland $\mathrm{I}$ : The influence of incubation $\mathrm{pH}$ on the activity of rat and human gut flora enzymes. Journal of applied bacteriology 1989, 66(5):433-437.

34. Gorbach S, Chang T-W, Goldin B: Successful treatment of relapsing Clostridium difficile colitis with Lactobacillus GG. The Lancet 1987, 330(8574):1519.

35. Lilly DM, Stillwell RH: Probiotics: growth-promoting factors produced by microorganisms. Science (New York, NY) 1965, 147(3659):747-748.

36. Moonga HB, Schoustra SE, Linnemann AR, Kuntashula E, Shindano J, Smid EJ: The art of mabisi production: A traditional fermented milk. PloS one 2019, 14(3):e0213541.

37. Schoustra SE, Kasase C, Toarta C, Kassen R, Poulain AJ: Microbial Community Structure of Three Traditional Zambian Fermented Products: Mabisi, Chibwantu and Munkoyo. PloS one 2013, 8(5).

38. Hamaimbo BT, Chileshe J, Marinda PA, Nyau V, Schoustra SE: The influence of dietary diversity and health status on the nutritional status of children aged 6-23 months in two districts of Zambia. Maternal and Child Health 2019, Under review.

39. Takagi R, Sasaki K, Sasaki D, Fukuda I, Tanaka K, Yoshida K-i, Kondo A, Osawa R: A single-batch fermentation system to simulate human colonic microbiota for high-throughput evaluation of prebiotics. PLoS One 2016, 11(8):e0160533.

40. De Baere S, Eeckhaut V, Steppe M, De Maesschalck C, De Backer P, Van Immerseel F, Croubels $S$ : Development of a HPLC-UV method for the quantitative determination of four short-chain fatty acids and lactic acid produced by intestinal bacteria during in vitro fermentation. Journal of Pharmaceutical and Biomedical Analysis 2013, 80:107-115.

41. Neter J, Kutner MH, Nachtsheim CJ, Wasserman W: Applied linear statistical models. Chicago: Irwin; 1996.

42. Graf D, Di Cagno R, Fåk F, Flint HJ, Nyman M, Saarela M, Watzl B: Contribution of diet to the composition of the human gut microbiota. Microbial Ecology in Health and Disease 2015, 26(1):26164.

43. Salonen A, De Vos WM: Impact of diet on human intestinal microbiota and health. Annual Review of Food Science and Technology 2014, 5(1):239-262. 
44. Justin Chileshe EFT, Sijmen E Schoustra, Karin J Borgonjen-van den Berg, Ray Handema, Bas J Zwaan and Inge D Brouwer: Potential contribution of cereal and milk based fermented foods to dietary nutrient intake of 1-5 years old children in Central province in Zambia. 2019.

45. Rajkumar H, Kumar M, Das N, Kumar SN, Challa HR, Nagpal R: Effect of Probiotic Lactobacillus salivarius UBL S22 and Prebiotic Fructo-oligosaccharide on Serum Lipids, Inflammatory Markers, Insulin Sensitivity, and Gut Bacteria in Healthy Young Volunteers:A Randomized Controlled Single-Blind Pilot Study. Journal of Cardiovascular Pharmacology and Therapeutics 2015, 20(3):289-298.

46. Mota de Carvalho N, Costa E, Silva S, Pimentel L, H. Fernandes T, Estevez Pintado M: Fermented Foods and Beverages in Human Diet and Their Influence on Gut Microbiota and Health; 2018.

47. Torii T, Kanemitsu K, Wada T, Itoh S, Kinugawa K, Hagiwara A: Measurement of short-chain fatty acids in human faeces using high-performance liquid chromatography: specimen stability. Annals of clinical biochemistry 2010, 47(5):447-452.

48. Sze MA, Topçuoğlu BD, Lesniak NA, Ruffin MT, Schloss PD: Fecal short-chain fatty acids are not predictive of colonic tumor status and cannot be predicted based on bacterial community structure. bioRxiv 2019:604678.

49. Gill PA, van Zelm MC, Muir JG, Gibson PR: Review article: short chain fatty acids as potential therapeutic agents in human gastrointestinal and inflammatory disorders. Alimentary Pharmacology \& Therapeutics 2018, 48(1):15-34.

50. Tamayo C: Clinical Research on Probiotics: The Interface between Science and Regulation. Clinical Infectious Diseases 2008, 46(Supplement_2):S101-S103.

51. Leroy JL, Ruel M, Habicht JP, Frongillo EA: Linear growth deficit continues to accumulate beyond the first $\mathbf{1 0 0 0}$ days in low- and middle-income countries: global evidence from $\mathbf{5 1}$ national surveys. J Nutr 2014, 144(9):1460-1466. 


\section{Tables and Figures}

Table 1: Primer sets used for qPCR with amplification parameters

\begin{tabular}{|c|c|c|c|}
\hline & & qPCR amplification & \\
\hline Name & Sequence & parameters & Melt curve \\
\hline All eubacteria & $\begin{array}{l}\text { F: ACTCCTACGGGAGGCAGCAGT } \\
\text { R: GTATTACCGCGGCTGCTGGCAC }\end{array}$ & $\begin{array}{l}95^{\circ} \mathrm{C}-3 \mathrm{~min}, 38 \mathrm{cycles} \text { of } \\
\left(95^{\circ} \mathrm{C}-30 \mathrm{sec} \& 54^{\circ} \mathrm{C}-30 \mathrm{sec}\right)\end{array}$ & $\begin{array}{l}95-15 \mathrm{sec}, 1 \% \text { decrease } 20 \mathrm{sec} \\
\text { starting at } 60^{\circ} \mathrm{C} \text { ending at } 95^{\circ} \mathrm{C} . \\
\text { final step at } 95^{\circ} \mathrm{C}-15 \mathrm{sec}\end{array}$ \\
\hline Bifidobacterium & $\begin{array}{l}\text { F: CTCCTGGAAACGGGTGG } \\
\text { R: GGTGTTCTTCCCGATATCTACA }\end{array}$ & $\begin{array}{l}50^{\circ} \mathrm{C}-2 \mathrm{~min}, 95^{\circ} \mathrm{C}-10 \mathrm{~min}, \\
38 \text { cycles of }\left(95^{\circ} \mathrm{C}-15 \mathrm{sec} \&\right. \\
\left.60^{\circ} \mathrm{C}-1 \mathrm{~min}\right)\end{array}$ & $\begin{array}{l}95-15 \mathrm{sec}, 1 \% \text { decrease } 20 \mathrm{sec} \\
\text { starting at } 60^{\circ} \mathrm{C} \text { ending at } 95^{\circ} \mathrm{C}- \\
\text { 30sec. final step at } 60^{\circ} \mathrm{C} 15 \mathrm{sec}\end{array}$ \\
\hline Bacteroides & $\begin{array}{l}\text { F: GGTGTCGGCTTAAGTGCCAT } \\
\text { R: CGGA(C/T)GTAAGGGCCGTGC }\end{array}$ & $\begin{array}{l}50^{\circ} \mathrm{C}-2 \mathrm{~min}, 95^{\circ} \mathrm{C}-10 \mathrm{~min}, \\
38 \text { cycles of }\left(95^{\circ} \mathrm{C}-15 \mathrm{sec} \&\right. \\
\left.60^{\circ} \mathrm{C}-1 \mathrm{~min}\right)\end{array}$ & $\begin{array}{l}95-15 \mathrm{sec}, 1 \% \text { decrease } 20 \mathrm{sec} \\
\text { starting at } 60^{\circ} \mathrm{C} \text { ending at } 95^{\circ} \mathrm{C}- \\
\text { 30sec. final step at } 60^{\circ} \mathrm{C} 15 \mathrm{sec}\end{array}$ \\
\hline Lactobacillus group & R: CACCGCTACACATGGAG & $\begin{array}{l}50^{\circ} \mathrm{C}-2 \mathrm{~min}, 95^{\circ} \mathrm{C}-10 \mathrm{~min}, \\
38 \mathrm{cycles} \text { of }\left(95^{\circ} \mathrm{C}-15 \mathrm{sec} \&\right. \\
\left.60^{\circ} \mathrm{C}-1 \mathrm{~min}\right)\end{array}$ & $\begin{array}{l}95-15 \mathrm{sec}, 1 \% \text { decrease } 20 \mathrm{sec} \\
\text { starting at } 60^{\circ} \mathrm{C} \text { ending at } 95^{\circ} \mathrm{C}- \\
\text { 30sec. final step at } 60^{\circ} \mathrm{C} 15 \mathrm{sec}\end{array}$ \\
\hline Enterococcus spp. & R: ACTCGTTGTACTTCCCATTGT & $\begin{array}{l}50^{\circ} \mathrm{C}-2 \mathrm{~min}, 95^{\circ} \mathrm{C}-10 \mathrm{~min}, \\
38 \text { cycles of }\left(95^{\circ} \mathrm{C}-15 \mathrm{sec} \&\right. \\
\left.60^{\circ} \mathrm{C}-1 \mathrm{~min}\right)\end{array}$ & $\begin{array}{l}95-15 \mathrm{sec}, 1 \% \text { decrease } 20 \mathrm{sec} \\
\text { starting at } 60^{\circ} \mathrm{C} \text { ending at } 95^{\circ} \mathrm{C}- \\
\text { 30sec. final step at } 60^{\circ} \mathrm{C} 15 \mathrm{sec}\end{array}$ \\
\hline
\end{tabular}




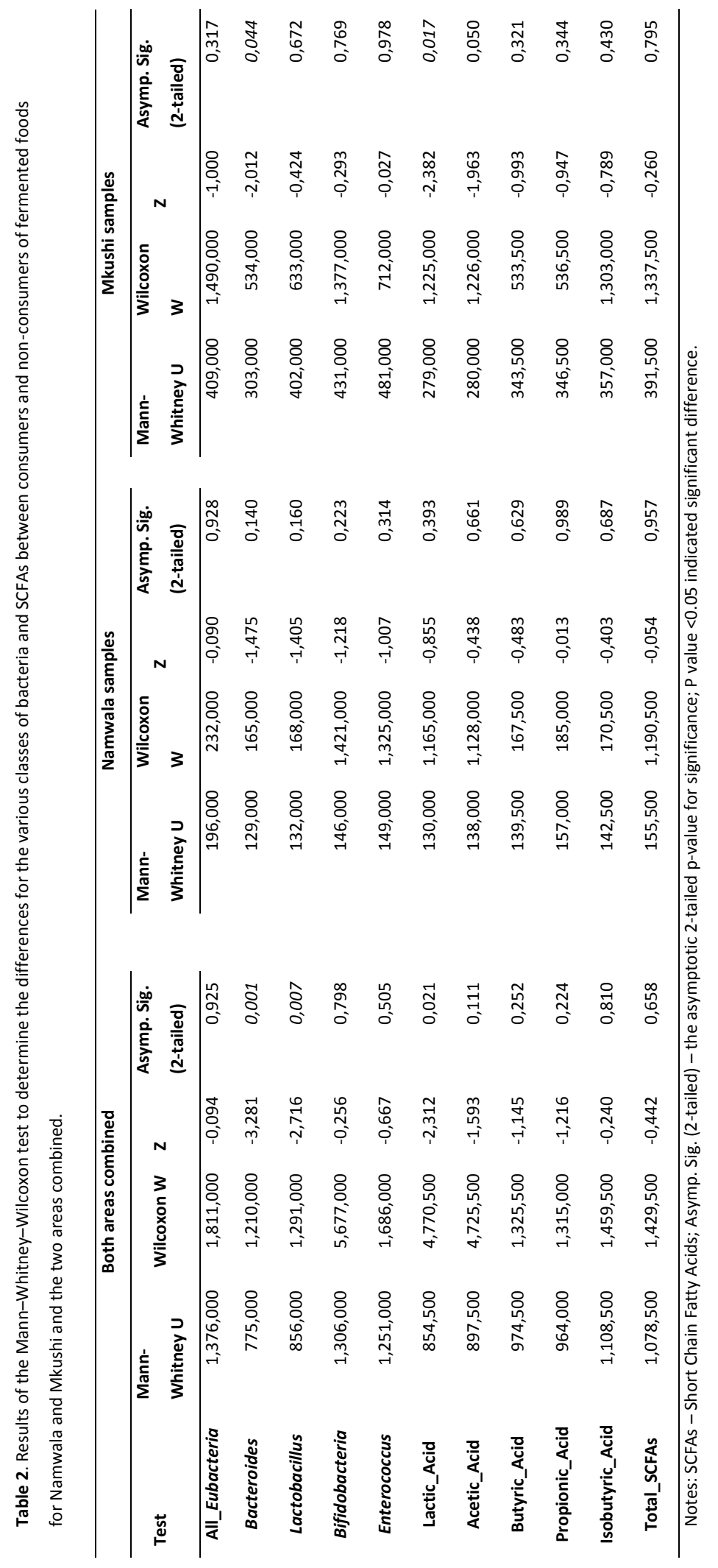



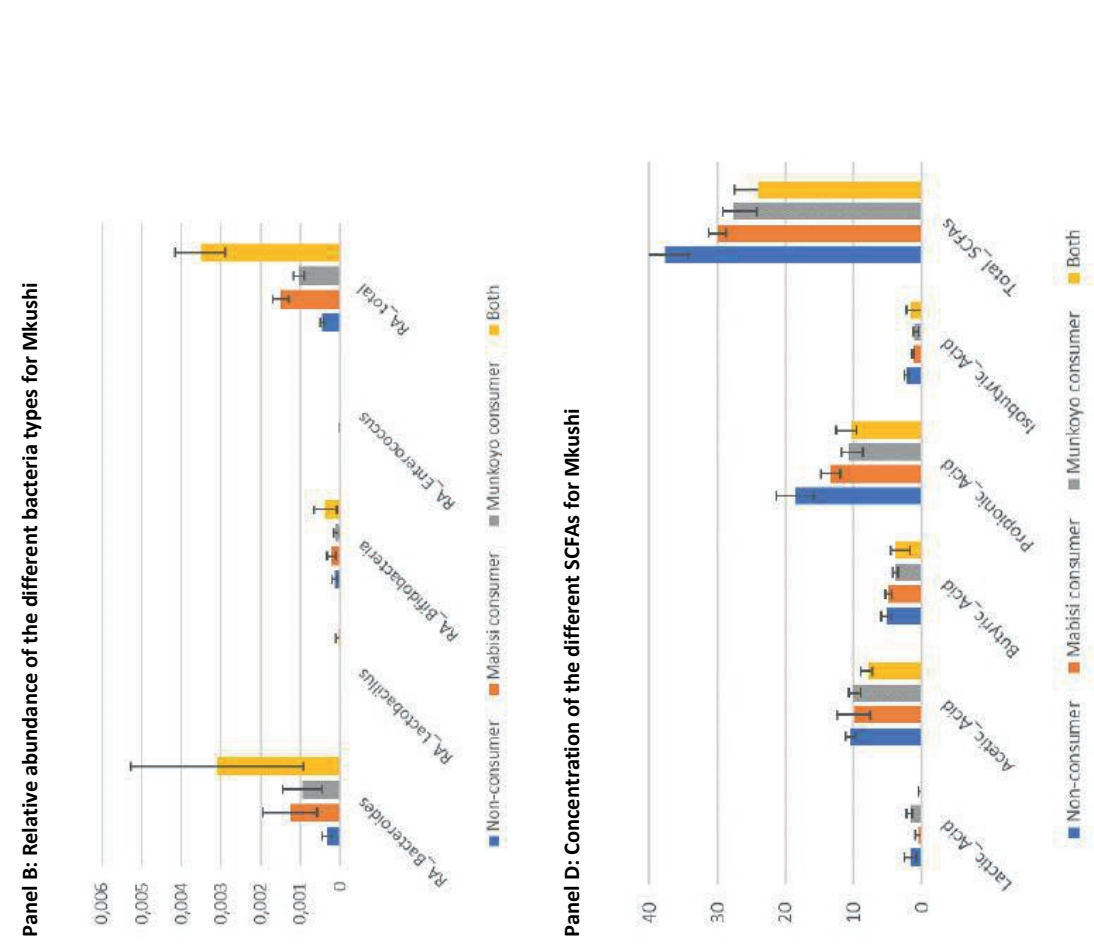

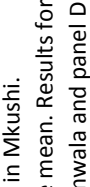

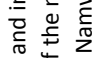

त

है

2 중

劳跑

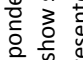

跣

ह

힌

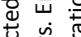

可

崩

产

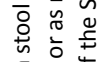

동

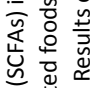

哉

比㐫

要常市

范

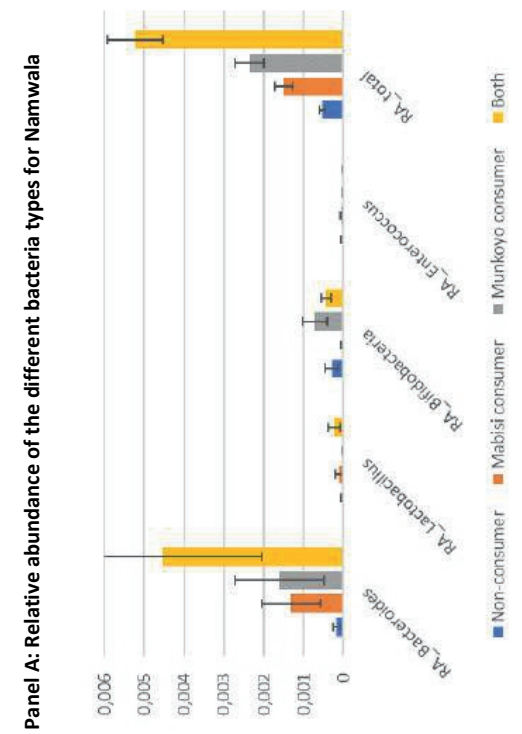

政

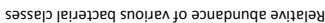


Supplementary Table 3: General Linear Regression (GLS) Tests ${ }^{a}$ for effect of different dietary intake, morbidity, socio-demographic variable on dependent variables gut bacteria (Bacteroides, Lactobacillus, Bifidobacteria and Enterococcus).

\begin{tabular}{|c|c|c|c|c|c|c|c|c|}
\hline \multicolumn{2}{|l|}{ Effect } & \multirow{2}{*}{\begin{tabular}{|l} 
Value \\
0.158 \\
\end{tabular}} & \multirow{2}{*}{$\begin{array}{l}\mathrm{F} \\
1.924^{\mathrm{b}}\end{array}$} & \multirow{2}{*}{$\begin{array}{l}\text { Hypothesis } \\
\text { df } \\
4\end{array}$} & \multirow{2}{*}{$\begin{array}{l}\text { Error } \\
\text { df } \\
41\end{array}$} & \multirow{2}{*}{$\begin{array}{l}\text { Sig. } \\
0.125\end{array}$} & \multirow{2}{*}{$\begin{array}{l}\text { Partial Eta } \\
\text { Squared } \\
0.158\end{array}$} & \multirow{2}{*}{$\begin{array}{l}\text { Observed } \\
\text { Powerd }^{\text {O }} \\
0.531\end{array}$} \\
\hline Intercept & Pillai's Trace & & & & & & & \\
\hline Childs_age & Pillai's Trace & 0.204 & $2.628^{b}$ & 4 & 41 & 0.048 & 0.204 & 0.684 \\
\hline HAZ & Pillai's Trace & 0.261 & $3.618^{b}$ & 4 & 41 & 0.013 & 0.261 & 0.834 \\
\hline Level_of_education & Pillai's Trace & 0.13 & $1.526^{\mathrm{b}}$ & 4 & 41 & 0.212 & 0.13 & 0.43 \\
\hline Family_size & Pillai's Trace & 0.17 & $2.103^{b}$ & 4 & 41 & 0.098 & 0.17 & 0.573 \\
\hline Source_of_drinking_water & Pillai's Trace & 0.118 & $1.365^{\mathrm{b}}$ & 4 & 41 & 0.263 & 0.118 & 0.387 \\
\hline Child_deworming & Pillai's Trace & 0.047 & $.504^{b}$ & 4 & 41 & 0.733 & 0.047 & 0.158 \\
\hline Illness & Pillai's Trace & 0.179 & $2.235^{b}$ & 4 & 41 & 0.082 & 0.179 & 0.603 \\
\hline Malaria & Pillai's Trace & 0.267 & $3.736^{b}$ & 4 & 41 & 0.011 & 0.267 & 0.847 \\
\hline Diarrhoea & Pillai's Trace & 0.18 & $2.247^{b}$ & 4 & 41 & 0.081 & 0.18 & 0.606 \\
\hline Vomiting & Pillai's Trace & 0.05 & $.542^{\mathrm{b}}$ & 4 & 41 & 0.705 & 0.05 & 0.167 \\
\hline Fever & Pillai's Trace & 0.024 & $.252^{\mathrm{b}}$ & 4 & 41 & 0.907 & 0.024 & 0.099 \\
\hline Other_illness & Pillai's Trace & 0.083 & $.934^{\mathrm{b}}$ & 4 & 41 & 0.454 & 0.083 & 0.269 \\
\hline Monthly_food_expenditure & Pillai's Trace & 0.126 & $1.473^{b}$ & 4 & 41 & 0.228 & 0.126 & 0.416 \\
\hline Monthly_non_food_expenditure & Pillai's Trace & 0.152 & $1.841^{b}$ & 4 & 41 & 0.139 & 0.152 & 0.511 \\
\hline Complementary_food & Pillai's Trace & 0.077 & $.861^{\mathrm{b}}$ & 4 & 41 & 0.496 & 0.077 & 0.25 \\
\hline Domestic_animals & Pillai's Trace & 0.227 & $3.014^{b}$ & 4 & 41 & 0.029 & 0.227 & 0.751 \\
\hline FG1 (Grains, roots and tubers) & Pillai's Trace & 0.007 & $.073^{\mathrm{b}}$ & 4 & 41 & 0.99 & 0.007 & 0.063 \\
\hline Maize_meal_porridge & Pillai's Trace & 0.09 & $1.015^{b}$ & 4 & 41 & 0.411 & 0.09 & 0.291 \\
\hline Rice & Pillai's Trace & 0.075 & $.831^{\mathrm{b}}$ & 4 & 41 & 0.513 & 0.075 & 0.242 \\
\hline Cereal_blend & Pillai's Trace & 0.035 & $.372^{\mathrm{b}}$ & 4 & 41 & 0.827 & 0.035 & 0.126 \\
\hline
\end{tabular}




\begin{tabular}{|c|c|c|c|c|c|c|c|c|}
\hline Sweet_potato & Pillai's Trace & 0.047 & $.500^{\mathrm{b}}$ & 4 & 41 & 0.736 & 0.047 & 0.157 \\
\hline Irish_potato & Pillai's Trace & 0.027 & $.283^{\mathrm{b}}$ & 4 & 41 & 0.887 & 0.027 & 0.106 \\
\hline Cassava & Pillai's Trace & 0.104 & $1.189^{b}$ & 4 & 41 & 0.33 & 0.104 & 0.339 \\
\hline Wheat & Pillai's Trace & 0.118 & $1.376^{b}$ & 4 & 41 & 0.259 & 0.118 & 0.39 \\
\hline FG2 (Legumes and nuts) & Pillai's Trace & 0.066 & $.725^{b}$ & 4 & 41 & 0.58 & 0.066 & 0.214 \\
\hline Beans & Pillai's Trace & 0.042 & $.453^{b}$ & 4 & 41 & 0.77 & 0.042 & 0.145 \\
\hline Soy_beans & Pillai's Trace & 0.154 & $1.861^{\mathrm{b}}$ & 4 & 41 & 0.136 & 0.154 & 0.516 \\
\hline Groundnuts & Pillai's Trace & 0.096 & $1.087^{b}$ & 4 & 41 & 0.376 & 0.096 & 0.311 \\
\hline Cowpeas & Pillai's Trace & 0.074 & $.820^{\mathrm{b}}$ & 4 & 41 & 0.52 & 0.074 & 0.239 \\
\hline Bambara_nuts & Pillai's Trace & 0.059 & $.647^{b}$ & 4 & 41 & 0.632 & 0.059 & 0.194 \\
\hline FG3 (vitamin A rich vegetables) & Pillai's Trace & 0.089 & $.996^{b}$ & 4 & 41 & 0.42 & 0.089 & 0.286 \\
\hline Rape & Pillai's Trace & 0.142 & $1.694^{b}$ & 4 & 41 & 0.17 & 0.142 & 0.473 \\
\hline Spinach & Pillai's Trace & 0.216 & $2.819^{b}$ & 4 & 41 & 0.037 & 0.216 & 0.719 \\
\hline Chibwabwa & Pillai's Trace & 0.085 & $.957^{\mathrm{b}}$ & 4 & 41 & 0.441 & 0.085 & 0.276 \\
\hline Bondwe & Pillai's Trace & 0.122 & $1.422^{b}$ & 4 & 41 & 0.244 & 0.122 & 0.402 \\
\hline Sweetpotato_leaves & Pillai's Trace & 0.107 & $1.225^{b}$ & 4 & 41 & 0.315 & 0.107 & 0.349 \\
\hline Other & Pillai's Trace & 0.147 & $1.773^{b}$ & 4 & 41 & 0.153 & 0.147 & 0.494 \\
\hline Mango & Pillai's Trace & 0.289 & $4.176^{b}$ & 4 & 41 & 0.006 & 0.289 & 0.888 \\
\hline $\begin{array}{l}\text { FG4 (other fruits and } \\
\text { vegetables) }\end{array}$ & Pillai's Trace & 0.297 & $4.323^{b}$ & 4 & 41 & 0.005 & 0.297 & 0.9 \\
\hline Banana & Pillai's Trace & 0.147 & $1.761^{b}$ & 4 & 41 & 0.155 & 0.147 & 0.49 \\
\hline Lemon & Pillai's Trace & 0.086 & $.968^{\mathrm{b}}$ & 4 & 41 & 0.436 & 0.086 & 0.279 \\
\hline FG5 (Dairy products) & Pillai's Trace & 0.06 & $.650^{\mathrm{b}}$ & 4 & 41 & 0.63 & 0.06 & 0.194 \\
\hline FG6 (eggs) & Pillai's Trace & 0.14 & $1.670^{b}$ & 4 & 41 & 0.176 & 0.14 & 0.467 \\
\hline
\end{tabular}




\begin{tabular}{|c|c|c|c|c|c|c|c|c|}
\hline Chicken_eggs & Pillai's Trace & 0.127 & $1.495^{b}$ & 4 & 41 & 0.221 & 0.127 & 0.421 \\
\hline Fowl_eggs & Pillai's Trace & 0.24 & $3.245^{b}$ & 4 & 41 & 0.021 & 0.24 & 0.786 \\
\hline FG7 (flesh foods) & Pillai's Trace & 0.105 & $1.205^{b}$ & 4 & 41 & 0.323 & 0.105 & 0.343 \\
\hline Fish & Pillai's Trace & 0.078 & $.870^{\mathrm{b}}$ & 4 & 41 & 0.49 & 0.078 & 0.252 \\
\hline Chicken & Pillai's Trace & 0.096 & $1.082^{b}$ & 4 & 41 & 0.378 & 0.096 & 0.31 \\
\hline Beef & Pillai's Trace & 0.281 & $4.004^{b}$ & 4 & 41 & 0.008 & 0.281 & 0.873 \\
\hline Pork & Pillai's Trace & 0.032 & $.341^{\mathrm{b}}$ & 4 & 41 & 0.849 & 0.032 & 0.119 \\
\hline Goat & Pillai's Trace & 0.097 & $1.099^{b}$ & 4 & 41 & 0.37 & 0.097 & 0.314 \\
\hline Organ meat & Pillai's Trace & 0.031 & $.333^{b}$ & 4 & 41 & 0.854 & 0.031 & 0.117 \\
\hline FG8 & Pillai's Trace & 0.253 & $3.477^{b}$ & 4 & 41 & 0.016 & 0.253 & 0.817 \\
\hline Oils & Pillai's Trace & 0.232 & $3.088^{b}$ & 4 & 41 & 0.026 & 0.232 & 0.763 \\
\hline Fats & Pillai's Trace & 0.013 & $.135^{\mathrm{b}}$ & 4 & 41 & 0.969 & 0.013 & 0.075 \\
\hline Fresh_milk & Pillai's Trace & 0.145 & $1.733^{b}$ & 4 & 41 & 0.161 & 0.145 & 0.483 \\
\hline Mabisi & Pillai's Trace & 0.741 & 2.001 & 20 & 176 & 0.009 & 0.185 & 0.982 \\
\hline Munkoyo_Chibwantu & Pillai's Trace & 0.093 & $1.057^{b}$ & 4 & 41 & 0.390 & 0.093 & 0.303 \\
\hline \multirow[t]{2}{*}{ Mabisi*Munkoyo_Chibwantu } & Pillai's Trace & 0.383 & 1.574 & 12 & 129 & 0.107 & 0.128 & 0.803 \\
\hline & Roy's Largest Root & 0.249 & $2.673^{c}$ & 4 & 43 & 0.045 & 0.199 & 0.695 \\
\hline
\end{tabular}

a. Design: Intercept + Childs_age + HAZ + Level_of_education + Family_size + Source_of_drinking_water + Child_deworming + IIlness + Malaria + Diarrhoea + Vomiting + Fever + Other_illness + Monthly_food_expenditure + Monthly_non_food_expenditure +

Complementary_food + Domestic_animals + FG1_Grains + Maize_meal_porridge + Rice + Cereal_blend + Sweet_potato + Irish_potato + Cassava + Wheat + FG2_Legumes + Beans + Soy_beans + Groundnuts + Cowpeas + Bambara_nuts + FG3 + Rape + Spinach + Chibwabwa + Bondwe + Sweetpotato_leaves + Other + Mango + FG4 + Banana + Lemon + FG5 + FG6 + Chicken_eggs + Fowl_eggs + FG7 + Fish + Chicken + Beef + Pork + Goat + Organ + FG8 + Oils + Fats + Fresh_milk + Mabisi + Munkoyo_Chibwantu + Mabisi * Munkoyo_Chibwantu

\section{b. Exact statistic}

c. The statistic is an upper bound on $\mathrm{F}$ that yields a lower bound on the significance level.

d. Computed using alpha $=.05$

$P$ values highlighted in red indicate significance $(<0.05)$ 
Supplementary Table 4: Socio-economic and demographic characteristics of the study population by study site (District) [38]

\begin{tabular}{|c|c|c|c|}
\hline Characteristic & $\begin{array}{c}\text { Namwala } \\
\text { n (\%) }\end{array}$ & $\begin{array}{c}\text { Mkushi } \\
\text { n (\%) }\end{array}$ & p-value \\
\hline Gender of the child & & & 0.449 \\
\hline Female & $56(52)$ & $49(47)$ & \\
\hline Male & $52(48)$ & $56(53)$ & \\
\hline Geographic setting & & & 0.401 \\
\hline Rural & $79(73.1)$ & $82(78.1)$ & \\
\hline Urban & $29(26.9)$ & $23(21.9)$ & \\
\hline Marital status of caregiver & & & 0.047 \\
\hline Married & $71(66)$ & $83(79)$ & \\
\hline Not married & $37(34)$ & $22(21)$ & \\
\hline Level of education of caregiver & & & 0.006 \\
\hline None & $4(4)$ & $4(4)$ & \\
\hline Primary & $55(51)$ & $31(30)$ & \\
\hline Secondary & $48(44)$ & $64(61)$ & \\
\hline Tertiary & $1(1)$ & $6(6)$ & \\
\hline Household monthly food expenditure (ZMW) & & & 0.047 \\
\hline$<500$ & $72(67)$ & $52(50)$ & \\
\hline $501-1000$ & $28(26)$ & $35(33)$ & \\
\hline $1001-2500$ & $6(6)$ & $12(11)$ & \\
\hline$>2500$ & $2(2)$ & $6(6)$ & \\
\hline Source of drinking water & & & 0.000 \\
\hline Stream & $4(4)$ & $18(17)$ & \\
\hline Well & $36(33)$ & $26(25)$ & \\
\hline Borehole & $48(44)$ & $21(20)$ & \\
\hline Tap & $19(18)$ & $35(33)$ & \\
\hline Other & $1(1)$ & $1(1)$ & \\
\hline
\end{tabular}


Supplementary Table 5: Morbidity characteristics of the study population by study site (District)

\begin{tabular}{|c|c|c|c|}
\hline Characteristic & $\begin{array}{c}\text { Namwala } \\
\text { n (\%) }\end{array}$ & $\begin{array}{c}\text { Mkushi } \\
\text { n (\%) }\end{array}$ & p-value \\
\hline Disease presented in last 2 weeks & & & 0.069 \\
\hline No (absence) & $30(27.8)$ & $43(41.0)$ & \\
\hline Yes (presence) & $7872.2)$ & $61(58.1)$ & \\
\hline Malaria in the last 2 weeks & & & 0.086 \\
\hline No (absence) & $89(82.4)$ & $95(90.5)$ & \\
\hline Yes (presence) & $19(17.6)$ & $10(9.5)$ & \\
\hline Diarrhoea in the last 2 weeks & & & 0.993 \\
\hline No (absence) & $74(68.5)$ & $72(68.6)$ & \\
\hline Yes (presence) & $34(31.5)$ & $33(31.4)$ & \\
\hline Vomiting in the last weeks & & & 0.097 \\
\hline No (absence) & $92(85.2)$ & $97(92.4)$ & \\
\hline Yes (presence) & $16(14.8)$ & $8(7.6)$ & \\
\hline Fever in the last 2 weeks & & & 0.004 \\
\hline No (absence) & $50(46.3)$ & $69(65.7)$ & \\
\hline Yes (presence) & $58(53.7)$ & $36(34.3)$ & \\
\hline \multicolumn{4}{|l|}{ Other illnesses in the last 2 weeks e.g. } \\
\hline cough & & & 0.115 \\
\hline No (absence) & $88(81.5)$ & $76(72.4)$ & \\
\hline Yes (presence) & $20(18.5)$ & $29(27.6)$ & \\
\hline
\end{tabular}


Chapter 6 


\section{General discussion}

Justin Chileshe 


\section{General Discussion}

The research described in my thesis was carried out within the framework of fighting undernutrition early in life with its many undesirable outcomes later in life. My goal was to contribute to the reduction of inadequate dietary intake of undernutrition by exploring the nutritional and microbial potential of traditional fermented foods Mabisi and Munkoyo.

In this thesis my aims were as follows:

1. to elucidate the nutritional potential of fermented foods through modelling to show whether these foods can potentially fill nutrient gaps in the diet of children under five years old (improvement of diets);

2. to determine the nutritional aspects and microbial composition of the target traditional fermented beverages Mabisi and Munkoyo and whether microbes influence the level of nutrients in the two products (increase of nutritional content through fermentation); and,

3. to investigate the influence of the favourable bacteria in Mabisi and Munkoyo in shaping human gut microbiota towards more favourable composition which favourably impacts on nutritional status (beneficial effects on gut microbiota composition).

The approach addressing the first aim was to use 24-hour recall data on food intake of children under the age of five in a rural area (Mkushi) in Zambia for analyses using Optifood. Optifood is a programme that linearly models diets and develops food based dietary recommendations with and without fermented foods to determine the nutrient gaps and to estimate whether the nutrient gaps could be closed with these fermented foods. To address the second aim, we analysed samples of fermented foods (Mabisi and Munkoyo) to determine the nutrient composition and the microbial composition and assessed whether there is a relationship between these two. To address the third aim, we (a) used an in-vitro experiment to determine effect of these fermented foods on gut microbiota metabolism and (b) conducted a cross-sectional survey to determine the assess the effect of fermented foods consumption on gut microbiota composition and short chain fatty acids (SCFAs) concentrations in children 6- 24 month of age.

In this general discussion, I reflect on the findings from the scientific chapters and their implications for the conceptual framework that I presented in the general introduction of this thesis (Figure 1). I will also present the future perspectives of the research directions to broaden the scope of the thesis 
work. Finally, I return to the UNICEF framework of causes and consequences of undernutrition to outline how my results contribute to our understanding and mitigation of the immediate, underlying and basic causes and their short and long-term consequences.

\section{Results of this thesis: Mabisi and Munkoyo as vehicles for alleviation of undernutrition}

Undernutrition, especially stunting and micronutrient deficiencies, are major health challenges faced in Zambia and is compounded by high rates of infectious diseases. The relationship between infections disease and undernutrition is synergistic, each further compromising the outcome of the other [1]. Undernutrition compromises natural immunity leading to increased susceptibility to infections, more frequent and prolonged disease episodes, and increased severity of disease [2]. Similarly, infections can exacerbate or precipitate undernutrition through reduced appetite and food intake, nutrient malabsorption, nutrient loss or increased metabolic needs [3]. This therefore calls for concerted efforts in the fight of both undernutrition and infectious diseases. The central premise of my thesis is that traditional fermented foods offer an opportunity in the fight against undernutrition through increased nutrient intake and also in mitigating infections through the beneficial effects of fermenting bacteria on the gut microbiota. 
Undernutrition in Zambia

Inadequate dietary intake

Underlying causes

Diets do not meet requirements

Suboptimal health parameters

Opportunity: Local fermented foods to improve

diets and health parameters

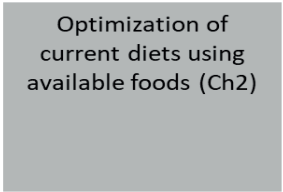

Food Based

Recommentations

including Mabisi and

Munkoyo show

positive impact on

diet quality of the two

products (Ch2)

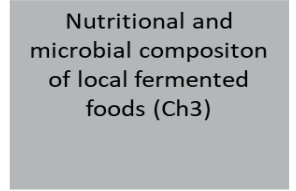

\section{Nutritional} composition of the two products is measured. Mabisi contributes to achieving EAR of essential nutrients (Ch3)

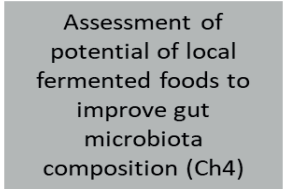

In vitro experiments show gut microbiota shifts to more

favourable composition and metabolic output after Mabisi and Munkoyo exposure (Ch4)

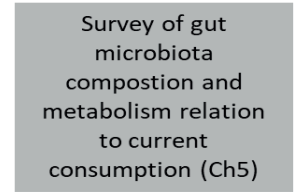

Cross sectional survey shows benefits of healthier gut microbiota composition as a result of Mabisi and Munkoyo consumption (Ch5)

\section{Better health of currently undernourished populations}

Figure 1. Diagram highlighting the main results of this thesis in the conceptual framework that was presented in the general introduction of this thesis. Here, I focus on the immediate causes of undernutrition in the UNICEF conceptual framework [4]. The research in this thesis sought to contribute to the improvement in nutrient intake through utilization of traditional fermented foods (Mabisi and Munkoyo) and their effect on the human gut microbiota. The main questions are in grey and the summary findings for the different scientific chapters are shown in pink.

\section{Improvements of diets}

My study using Optifood to model dietary intake data suggested that inclusion of Mabisi in the diets of children aged 1-5 years improves their nutrient intake, especially enhancing intake levels of the common problematic micronutrients calcium, iron, and zinc (Chapter 2). It supports the importance of targeting the local foods such as Mabisi and others in promoting dietary diversification to cartel the dependence on the monotonous (maize-based) diets.

Next, I measured the nutritional composition of Mabisi and Munkoyo, confirming that especially Mabisi can contribute to achieving improved nutrient intake of various important macro- and micro- 
nutrients (Chapter 3). For many of the locally available foods such as Mabisi and Munkoyo, nutritional data have been lacking impeding their consideration for inclusion in food based dietary guidelines. Therefore, the data generated on the nutritional content of the two fermented products are useful for inclusion in the food-based guidelines. When connecting results reported in Chapter $\mathbf{3}$ the nutritional composition of Mabisi and Munkoyo reported in this thesis with the calculated percentage contribution of the nutrients towards meeting the estimated average requirements (EARs) and the results of Chapter $\mathbf{2}$ on improved dietary intake based on current diets, my thesis provides strong support for promotion of mabisi to improve the quality of current diets.

I however recommend more research to especially incorporate determination of the nutritional and microbial aspects in the fermentation process for the raw materials and end-products to link nutritional value with fermenting microbes and conduct more genomic analysis for B vitamin production. Another important aspect to further explore is the bioavailability of the (micro)nutrients in the context of the achieved diets by for example estimating molar ratios of phytate to zinc, iron, and calcium in the diets.

\section{Increase of nutritional content through fermentation}

Mabisi was found to have higher nutrient values than Munkoyo except for some B vitamins such as vitamin B1, B3 and B6. This implies that Mabisi has a higher advantage than Munkoyo for making an impact in nutrient intake as was shown in Chapters $\mathbf{2}$ and $\mathbf{3}$. The increase in B vitamins and possible probiotic effect of Munkoyo makes it also a product that would be useful for regular consumption for improvement in dietary diversity.

\section{Beneficial effects on gut microbiota composition}

It was clearly shown that the consumers of fermented products had higher abundance of gut bacteria than the non-consumers and that the region with especially higher Mabisi consumption had higher gut bacteria abundance and better nutritional status. The potential probiotic effect of traditional fermented foods was assessed through an in-vitro laboratory system (Chapter 4). The set up allowed for analysis of metabolites (in my case short chain fatty acids, SCFAs) produced by the bacterial consortia from gut microbiota in response to addition of traditional fermented foods and for monitoring of shifts in abundance of Lactobacillus. Both Mabisi and Munkoyo were shown to increase the production of SCFAs and increasing abundance of Lactobacillus. Based on this work, levels of consumption may not be that important as both high and low levels of exposure generated probiotic effects. The type of traditional fermented food may also not be that important, as long as it contains a 
wide range of live bacteria. These results can therefore motivate and refine future nutrition intervention studies to assess the effect of the Mabisi and Munkoyo on gut microbiota and health outcomes in consumers.

In Chapter 5 the effect of Mabisi and Munkoyo/Chibwantu consumption on gut microbial composition and nutritional status in children 6-24 months old in rural Zambia was investigated. The children who consumed fermented beverages had relatively higher abundance of Bacteroides, Lactobacillus, Enterococcus and Bifidobacteria in their stool than the non-consumers. Namwala being a high area of Mabisi consumption also was found to have better nutrition status and consequently had higher copy numbers of the measured bacterial species than Mkushi. This study has elicited important data pointing to the potential of traditional fermented beverages Mabisi and Munkoyo consumption to impact on nutritional status. Thus, these results give an impetus to conduct controlled human feeding trials to support and confirm the impact of the traditional fermented beverages consumption on gut bacteria modulation and general possible interaction between human gut system - fermented beverages.

Taken together, the evidence that intake of Mabisi and Munkoyo contribute to alleviation of undernutrition in this thesis includes:

1. Improvement of diets: inclusion of Mabisi in diets of children under the age of five years old improves nutrient intake of most nutrients except iron and zinc;

2. Increase of nutritional content through fermentation: Mabisi has higher amounts of most nutrients but both products are good sources of $B$ vitamins. Through fermentation of the raw materials (raw milk for Mabisi and cereal for Munkoyo), the levels of B-vitamins can increase. Levels of increase likely depend on the exact composition of the microbial community used for fermentation;

3. Beneficial effects on gut microbiota composition: Mabisi consumption is associated with higher abundance of gut bacteria and also with better nutritional status among children that were part of a survey conducted in a rural area.

\section{Future directions and implementation}

Thus, in the framework of my project it is evident that the two products are good sources of nutrients and have potential probiotic effects to confer better health and nutritional status. In this section of my general discussion, I will describe how each of the three main findings summarised above could be implemented and elaborated further to maximize impact towards alleviating undernutrition. 


\section{Improvement of diets}

The Optifood modelling combined with the assessment of the nutritional composition of Mabisi and Munkoyo have shown that including these two products will improve diets. It will be important to repeat modelling of diets using data from the representative parts of the country in order to capture more nutrient rich foods and to include the cost aspect to obtain the food-based recommendations with a national outlook. More generally, it should be pointed out that while Mabisi and Munkoyo contribute to the improvement of diet quality, reducing nutrient deficiencies is a multifactorial problem.

\section{Bioavailability}

The assessment of nutritional composition of Mabisi and Munkoyo should be extended to include bioavailability of the nutrients. Fermentation is known for its ability to reduce phytate levels of raw materials. This should be measured directly, especially for Munkoyo that is cereal based, by measuring levels of phytate before and after fermentation. To estimate what the impact of reduced phytate levels would be on the full diets, all phytate in the diet should be measured [5] and molar ratios of phytate to zinc, iron and calcium should be estimated for Zambian diets in and excluding the effects of reduced phytate in Munkoyo as compared to maize. Lower molar ratios of phytate to the zinc, iron and calcium indicates a higher bioavailability of the trace elements. An older study in Malawi found high molar ratios of over 20 for phytate to zinc and to iron [6]. This may be similar in Zambia, underlining the importance of estimating bioavailability.

A further important issue regarding bioavailability is the interaction between caseins and iron in milk products, most likely including Mabisi. Casein is the main protein in milk and can complex iron molecules. This included the iron from the milk, but may extend to iron from other sources that are consumed simultaneously, for instance with Mabisi. Several studies have highlighted that especially children under the age of 24 months should avoid consumption of cow's milk together with other foods at the same time $[7,8]$. Fermentation may reduce the levels of casein from the raw milk, however, this reduction as well as the effects of Mabisi consumption on iron bioavailability in the diet should be investigated further.

\section{Adoption of Mabisi and Munkoyo by consumers}

Key messages need to be developed for nutrition education to the communities on the importance of different local foods, diversification of diets, and the fermented foods and their benefits. For Mabisi, at present products are exclusively produced in rural areas, with over $80 \%$ of households producing for own consumption, and around $20 \%$ of households selling excess product on nearby markets [9]. 
Availability of products in larger towns and urban areas is almost non-existent. Together with MSc students, I have conducted pilot interviews in large urban centres in Zambia targeting potential consumers from various socio-economic groups [10]. This revealed that $85 \%$ of urban consumers over all socio-economic groups would prefer traditional/artisanal Mabisi they already know through their (family) visits to rural areas over industrially processed and fermented dairy products. These potential consumers expressed concerns that Mabisi may be an unsafe product since no formal standards exist and the product has not been endorsed by the Zambia Bureau of Standards. Thus, Mabisi production should be formalized and approved by local standards bureaus to take away these concerns of urban consumers that would prevent them from adopting Mabisi. Upscaling of production would also increase overall availability. My thesis has contributed to the drafting of future research into upscaling of production, most notably taking into account the needs of current producers and ranges of urban consumers (Box 1).

More generally, diets need to be diversified, providing alternatives to the current maize dominated meals. Mabisi and Munkoyo could be options, these products are part of local culture of many Zambia's tribes. Education and promotion campaigns should tap into this potential, highlighting their nutritional benefits and aligning this to local tradition. Formal institutions such as the National Food and Nutrition Commission (NFNC) in Zambia and non-governmental organisations (NGOs) working on the promotion of better diets should be made aware of the nutritional potential of local foods such as Mabisi and Munkoyo.

\section{BOX 1. Bottom-up formalisation and upscaling of production}

Increase in supply would demand optimization of the current value chain of Mabisi and Munkoyo to extend current supply to larger urban centres. This would require upscaling of production and logistics of transportation, together with marketing. This upgrading of the value chain could be based out of local farmer groups or cooperatives. During field visits we also observed that local producers have started to form associations and cooperatives. Rural milk collection centres have looked into value addition by small-scale processing of raw milk for local sales to generate income and to prevent losses of uncollected milk.

When optimizing the value chains, special attention to the needs of current predominantly female producers is essential. Currently, these female producers generate livelihoods for their families though Mabisi production. Lifting production and sales from household level could cause a shift from the predominantly female producers in households to male-based small-scale production systems, 
potentially putting current producers/sellers out of business. Therefore, a focus on fostering and strengthening women entrepreneurship is needed. Producers and processors of many traditional foods including the ones we focus on at the moment are mostly women; optimizing the value chain generates opportunities for women entrepreneurship [11]. The social position of these women often hinders entrepreneurial competence development and behavior [12, 13]. A conscious gender approach in our research and outreach will lead to more participation in decision making, and will improve the social standing of women as entrepreneurs [14]. This will lead to higher incomes and performances that contribute to women's social and economic empowerment [15]. And the empowerment of woman may result in increased income and as a result increased nutritional status of their households[16].

\section{Increase of nutritional content through fermentation}

Fermentation has been recognised for its capacity to increase the nutritional value of the raw materials that the products are made out of. Well-known and well-studied are the effects of reduction in antinutritional factors such as indigestible saccharides and phytate that can limit the bioavailability of micronutrients.

The results of this thesis suggest that the microbial consortia in the fermentation of Mabisi and Munkoyo excrete B vitamins in the final products (Chapter $\mathbf{3}$ ). This analysis needs to be expanded, by systematically measuring levels of B vitamins before and after fermentation. Future study can assess the effect of variations in processing methods that exist for Mabisi [9]. Variations in processing have been shown to impact microbial community composition, this may in turn impact the levels of vitamins. The variations in microbial composition that I observed in my samples of Mabisi (Chapter 3) has inspired pilot studies in collaboration with an MSc thesis student for a study into the genetic potential of the microbes present in our product samples to produce vitamin B3 (Box 2).

\section{BOX 2. Genetics of Vitamin B3 production by microbes during fermentation}

Many prokaryotes used for food fermentation processes express the enzymes to synthesize water soluble vitamins. The organisms most known for this characteristic are lactic acid bacteria (LAB), and B vitamins - mainly B2, B3, B11 and B12 - being most commonly produced [17]. We used the Mabisi samples described in Chapter 3 for which we had assessed the variations in microbial composition as well as the nutritional composition. While microbial communities differ, they all yield a Mabisi product with a vitamin B3 content that is higher than the vitamin B3 content in raw milk. The levels 
of vitamin B3 in each Mabisi samples are different from each other, these differences could be explained by genetic differences at the level of microbial communities. If indeed the levels of vitamin B3 depend on microbial composition, conscious selection of a starter culture or to the selection of a production process will help to maximize the addition of vitamin B3 into the final product.

Vitamin B3 (also known as nicotinic acid or niacin) synthesis (Figure 2) starts from L-tryptophan or Laspartate, molecules which in a series of steps can be converted up to Vitamin B3. (Finglas, 2000; KEGG database, 2015). In this pilot study, we firstly asked whether we could detect the genes required for the final steps of the pathway within the microbial community ( $p n c B$ and $p u n A$ ) at DNA level, followed by a bacterial community wide RNA level gene expression analysis based on qPCR of the bacterial communities from the samples by placing them in fresh milk. We used the concentration of Vitamin B3 that was measured previously to draw inferences on a possible linkage between Vitamin B3 levels and gene expression of punA.
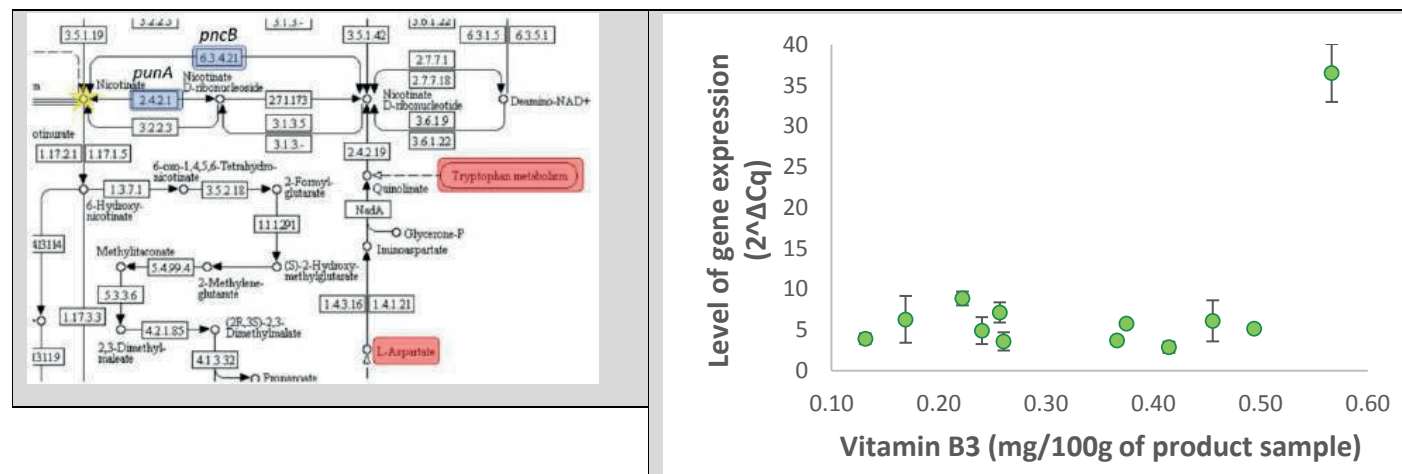

Figure 2. (left panel) Niacin synthesis pathway. Modification from KEGG database, 2015 [18]. Where precursors are marked in red, enzymes in blue and Niacin in yellow. (right panel) Observed levels of Vitamin B3 in the product samples as a function of gene expression levels of punA. The levels of Vitamin B3 were taken from the previous analysis of nutritional composition presented in Chapter 3.

The DNA based screen showed that all bacterial communities present at least one of the two last enzymes in the pathway to vitamin B3 production in at least one of the members of the bacterial community. The expression of these genes might depend on environmental stimuli such as other members of the community, their metabolites or enzymes in the media [19]. Differences in punA gene expression were observed at the RNA level. Following the general assumption that DNA is transcribed to RNA and eventually translated into a protein with a specific function [20], gene expression is expected to be proportional to vitamin B3 content. However, the data on vitamin B3 
were not obtained from the same experimental samples used for the RNA based gene expression analysis and the correlation should therefore be treated with much caution. Further, only gene expression levels of punA and not $p n c B$ were measured. Nevertheless, the results of this pilot study clearly highlight the genetic potential of the strains within the bacterial community to produce vitamin B3 and there is some indication that high levels of expression of these genes lead to higher levels of this vitamin. These results encourage future experiments that are more controlled and elaborate.

\section{Beneficial effects on gut microbiota composition}

The results presented in my thesis suggest that the interaction between microbes from the two traditional fermented products helps to shift the gut microbiota composition to a healthier composition for the host. In this way, Mabisi and Munkoyo can be considered as probiotics [21]. However, for a product to be labelled a probiotic, scientific evidence for the health benefit would have had to be documented. My experimental work included an in-vitro laboratory study and a crosssectional survey that included children under 24 months of age about their Mabisi and Munkoyo consumption. Both Mabisi and Munkoyo were found to confer beneficial effects by increasing the abundance of beneficial bacteria such as lactobacillus and higher concentrations of short chain fatty acids that are indicative of higher levels of abundance of bacteria that are considered beneficial. These results may be considered as a proxy for contributions towards better health of increased Mabisi and Munkoyo consumption and are thus highly encouraging. Using these insights, further study to elicit scientific evidence for health benefits of these products should be obtained through randomized controlled trials.

\section{Controlled nutrition intervention trials}

To determine probiotic effects of a product, there is need to carry out randomised controlled trials in humans with a health condition for which it can show health benefits. Studies have been done targeting for example diarrhoeal diseases caused by treatment with antibiotics. The set-up of such a controlled trial focusing on Mabisi could be to recruit three groups of children; group 1: Treatment group (Mabisi), group 2: milk group (non-fermented) and group 3: probiotic (yoghurt) with known effects. Study participants would consume their respective product twice a day for three months. The study would have assessment at baseline and end-line, including gut microbiota composition, age, weight, height and screening for any illnesses or symptoms thereof. 
This study has various foreseen challenges, such as how to manage the batches of fermented products for feeding children during the follow up period of three months. Keeping children in the study may be difficult including loss to follow up. Possible solutions are to find suppliers for weekly supplies making sure that the products are of the same quality by monitoring certain parameters such as $\mathrm{pH}$ and the taste of the products on a daily basis. Creating an incentive for keeping the children in the study team is another alternative, for example having a teacher for the groups of children with games that are attractive to the children. Community mobilisation would need to be done in a manner that motivates the parents to have their children participate. Having regular updates on any data generated to the communities. The need to recruit children whose parents are resident of the community and avoiding those that travel regularly would be of help. Involving locals in the study is another incentive that may promote confidence in the community.

My results on how Mabisi and Munkoyo may affect the gut microbiota of consumers has helped to design a follow-up project that focuses on Infant and Young Child Feeding programs. The University Teaching Hospital in Lusaka has introduced the use of milk-based therapeutic feeds that have been used to reduce mortality in children with severe acute malnutrition (SAM). Among the major complication seen in SAM and other forms of malnutrition is the unhealthy composition of the gut microbiota. In response to low tolerance of regular F-100 feed by undernourished children in Zambia, the teaching hospital has started exploring the use of fermented versions of F-100 and other fermented foods.

\section{Mabisi and Munkoyo as vehicles to mitigate underlying and basic causes and consequences of undernutrition}

As highlighted in this general discussion, my thesis was inspired by seeking potential solutions to the immediate causes of (maternal and child) undernutrition as defined by UNICEF (Figure 3). The results of this thesis can indeed be used to complement mitigation of several of the underlying and basic causes. One of the underlying causes of undernutrition is lack of household food security. The results of this thesis have shown the (nutritional) value of two traditional foods that are part of tradition and culture and that there is great interest of consumers in larger urban areas for these products should they be lifted out of the informal sector. The fact that the potential associated to these products in improving diets has now been formally demonstrated could help formal institutions to promote these products among consumers and help setting up programs for upscaling and formalisation (see e.g. Box 1). This will not only directly help consumers, it will also generate livelihoods for local producers, which will promote local household food security. 


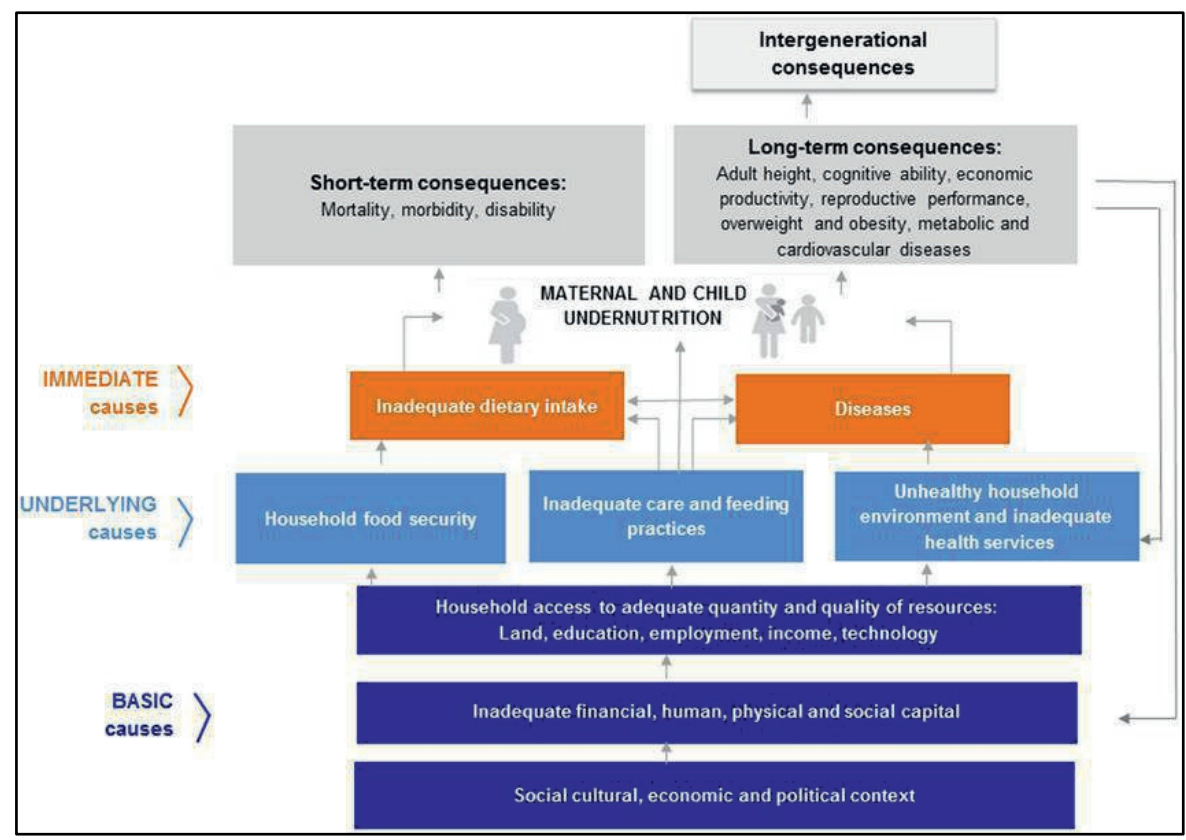

Figure 3: The UNICEF conceptual framework of causes and consequences of undernutrition. The black arrows show that the consequences of undernutrition can feed back to the underlying and basic causes of undernutrition, perpetuating the cycle of undernutrition, poverty and inequities [22].

Another basic cause of undernutrition is the lack of access to technology. Production of Mabisi and Munkoyo is low-cost and uses low-technology. Upscaling of the production is currently impeded by lack of technological infrastructure; however, this infrastructure is within reach of current producers through the formation of farmer cooperatives. The results of this thesis provide an incentive of farmer cooperatives and policy makers to further promote Mabisi and Munkoyo production and to put in place processing facilities based at these farmer cooperatives. Other basic cause is the cultural and political context. This thesis has underlined that traditional foods embedded in local culture do not only form part of a cultural heritage, they can also significantly contribute to promoting nutrition. This could help to shift the mind-set that local products are inferior to globalized products and can boost cultural self-esteem as well as nutrition. In terms of the political context, this would, together with other recently published work on Mabisi $[9,23]$ be used by formal institutions such as the Zambia Bureau of Standards to formulate standards and to facilitate Mabisi and Munkoyo on formal markets. 


\section{Traditional fermented foods as local foods}

While this thesis specifically focussed on traditional fermented foods, the results also apply to local foods in general. Local foods have several advantages, such as that they are culturally embedded and are often easily available for rural consumers as they are predominantly grown (or gathered) by local (small-scale) producers. One specific opportunity to improve the quality of diets is to make better use of local and traditional foods. These foods have not always been considered or visible in formal food markets or in national nutrition policies, often because of a lack of information. As was the case for Mabisi and Munkoyo, the nutritional value of local products is often not known, primarily because it concerns a large number of crops and animal food products commonly produced and processed using non-standardized and varying methods, resulting in large variations in nutritional content. Thus, it is not easy to classify and understand local foods, let alone including them in diet optimization models. When optimizing and formalizing local food products into the formal market, the aspect of optimization of nutritional potential should therefore be one of the prominent factors that needs to be taken into account

This knowledge can then be used to formulate criteria and legislation that allow current small holders to gain access to formal markets, which is a powerful way to facilitate for bottom-up upscaling. Further, adding nutrition to the food security discussion introduces current practitioners to the integration of nutrition value into the agricultural-food chain. This may inspire initiatives of technological upscaling that incorporates nutritional status of consumers and nutritional potential of upscaled and formalized products. In addition, the appetite for local foods among consumers also needs to be enhanced. More consumer awareness is needed on the (nutritional) value of local food crops, and in this context of convenient (you can buy it around the corner) and (lower) prices are also important.

The work described in my thesis shows that the goal of contributing to improvement of people's lives through dietary intake improvements using artisanal method of fermentation could be realised. Particularly the work in this thesis has influenced the way I have looked at bacteria and how it can positively affect diets and our lives. My work highlights the need to explore locally available and culturally accepted foods more so the fermented foods such as Mabisi and Munkoyo in the fight against undernutrition. However further work to generate more evidence for the formalization of our products Mabisi and Munkoyo needs to go hand in hand with key messages for the current and could-be consumers on their health benefits. 


\section{References}

1. Scrimshaw NS, SanGiovanni JP: Synergism of nutrition, infection, and immunity: an overview. The American journal of clinical nutrition 1997, 66(2):464S-477S.

2. Cunningham-Rundles S, McNeeley DF, Moon A: Mechanisms of nutrient modulation of the immune response. Journal of Allergy and Clinical Immunology 2005, 115(6):1119-1128.

3. Uchendu FN, Atinmo T: The silent and neglected crisis of malnutrition: scientific evidence for taking decisive action. Global Journal of Health Science 2011, 3(1):193.

4. Unicef: Improving child nutrition: the achievable imperative for global progress. New York: UNICEF 2013:1-114.

5. Ma G, Li Y, Jin Y, Zhai F, Kok FJ, Yang X: Phytate intake and molar ratios of phytate to zinc, iron and calcium in the diets of people in China. European Journal Of Clinical Nutrition 2006, 61:368.

6. Ferguson EL, Gibson RS, Thompson LU, Ounpuu S, Berry M: Phytate, zinc, and calcium contents of $\mathbf{3 0}$ East African foods and their calculated phytate:Zn, Ca:phytate, and [Ca][phytate]/[Zn] molar ratios. Journal of Food Composition and Analysis 1988, 1(4):316-325.

7. Ziegler EE: Consumption of cow's milk as a cause of iron deficiency in infants and toddlers. Nutrition reviews 2011, 69(suppl_1):S37-S42.

8. Thorsdottir I, Thorisdottir AV: Whole Cow's Milk in Early Life.

9. Moonga HB, Schoustra SE, Linnemann AR, Kuntashula E, Shindano J, Smid EJ: The art of mabisi production: A traditional fermented milk. PloS one 2019, 14(3):e0213541.

10. Ven Lvd: Consumer perspective of Mabisi, MSc Thesis, Wageningen UR. 2018.

11. Amine LS, Staub KM: Women entrepreneurs in sub-Saharan Africa: An institutional theory analysis from a social marketing point of view. Entrepreneurship \& Regional Development 2009, 21(2):183211.

12. Maas J, Seferiadis AA, Bunders JFG, Zweekhorst MBM: Bridging the disconnect: how network creation facilitates female Bangladeshi entrepreneurship. International Entrepreneurship and Management Journal 2014, 10(3):457-470.

13. Poggesi S, Mari M, Vita L: What's new in female entrepreneurship research? Answers from the literature. International Entrepreneurship and Management Journal 2016, 12(3):735-764.

14. Fuentes-Fuentes MdM, Bojica AM, Ruiz-Arroyo M: Entrepreneurial orientation and knowledge acquisition: effects on performance in the specific context of women-owned firms. International Entrepreneurship and Management Journal 2015, 11(3):695-717.

15. Mozumdar L: Entrepreneurship Against The Tide. Business performance of women entrepreneurs in a constrained environment. A survey in Bangladesh. Wageningen University, PhD thesis under supervision of G Hagelaar, VC Materia, SWF Omta; 2018.

16. Van den Bold M, Quisumbing AR, Gillespie S: Women s Empowerment and Nutrition: An Evidence Review, vol. 1294: Intl Food Policy Res Inst; 2013.

17. Burgess CM, Smid EJ, van Sinderen D: Bacterial vitamin B2, B11 and B12 overproduction: an overview. International journal of food microbiology 2009, 133(1-2):1-7.

18. Kanehisa M, Sato $Y$, Kawashima M, Furumichi M, Tanabe M: KEGG as a reference resource for gene and protein annotation. Nucleic acids research 2015, 44(D1):D457-D462.

19. Wintermute EH, Silver PA: Dynamics in the mixed microbial concourse. Genes \& development 2010, 24(23):2603-2614.

20. Clark D, Pazdernik N: Molecular biology. Waltham. In.: USA: Elsevier Inc; 2013.

21. WHO F: Evaluation of health and nutritional properties of powder milk and live lactic acid bacteria. Food and Agriculture Organization of the United Nations and World Health Organization Expert Consultation Report 2001:1-34.

22. Fund UNCs: Improving Child Nutrition: The achievable imperative for global progress. 2013.

23. Groenenboom AE: Microbial community dynamics in traditionally fermented milk. Wageningen: Wageningen University; 2019. 


\section{Summary}

The widespread societal, development and health problem of undernutrition in many developing countries motivated my research described in this thesis. Despite past interventions and economic developments, many developing countries still face high levels of undernutrition; especially stunting (linear growth failure), deficiencies in zinc, vitamins A and B12, and folate, mainly in children under the age of five years. Some of the current and proposed interventions to improve nutritional status include dietary diversification, sugar fortification with vitamin A, high dose vitamin A capsule distribution to under five children twice every year, maize meal fortification, and others. Dietary diversification and improvement in the food preparation methods such as fermentation are potential means with potential to improve availability of nutrients especially for vulnerable populations. For this thesis, the focus is on Zambia, which is one of the most undernourished countries in the world, with $48 \%$ of the population considered undernourished. As Zambia is dependent on mainly maize as a staple food with lower consumption of animal food sources, diversification of diets presents an opportunity to combat undernutrition in this population. Within this, the traditional use of fermented foods presents an opportunity for improvement in nutrient intake in the population especially of children below the age of five years. My thesis explores this potential.

My research has three central aims. (1) Elucidating the nutritional potential of the traditional fermented foods Mabisi and Munkoyo to complement the diet of vulnerable groups such as children under five years old (Chapter 2). (2) Determining the nutritional aspects and microbial composition of the target traditional fermented beverages Mabisi and Munkoyo and whether microbes influence the level of nutrients in the two products (Chapter 3). (3) Understanding the influence of the favourable bacteria in the products in shaping human gut microbiota towards more favourable composition and its impact on nutritional status (Chapters 4 and $\mathbf{5}$ ).

In Chapter $\mathbf{2}$ I describe the potential contribution of Mabisi and Munkoyo to nutrient intake in children under the age of five years using Optifood, a linear programming software that was developed by WHO and partners. Secondary dietary intake data collected using 24-hour recall method was modeled to develop food based recommendations (FBRs), for children aged 1-3 and 4-5 years in Mkushi district of Zambia. Three scenarios per age group were modeled to determine weekly food-based recommendations based on: (1) food based recommendations with the local available foods, (2) food based recommendations with inclusion of Mabisi, a fermented milk beverage, and (3) food based recommendations with inclusion of Munkoyo, a cereal fermented beverage. The scenarios were compared to assess whether food based recommendations with the addition of Mabisi and/or Munkoyo achieved better nutrient intake. FBRs based on only locally available non-fermented foods 
did not meet $\geq 70 \%$ of recommended nutrient intake (RNI) for calcium, fat, iron and zinc. The addition of Munkoyo to the FBRs did not reduce the number of problem nutrients, but after adding Mabisi to the FBR's only iron (67\% of RNI) remained a problem nutrient in the 1-3 year age group and only zinc (67\% of RNI) remained a problem nutrient in the $4-5$ year age group. Mabisi, a fermented milk product in combination with the local food pattern is a good additional source of nutrients for these age groups. However, additional nutrition sensitive and cost-effective measures would still be needed to improve nutrient intake, especially that of iron and zinc.

Chapter 3 describes the results obtained from laboratory analysis of Mabisi and Munkoyo samples collected during a cross sectional study in Mkushi to determine their nutritional composition and microbial communities. It was hypothesized that Mabisi and Munkoyo each contain distinct microbial communities and that nutritional composition depends on microbial composition. With respect to the variation in microbial community structure, I therefore addressed whether the use of different raw materials can explain the variation in the structure of microbial communities, and if variation in nutritional composition can be explained by differences in microbial community structure. Here, we characterized the nutritional composition and microbial community composition of Mabisi and Munkoyo. We found that the two products are different with respect to the nutritional and the microbial composition. Mabisi was found to have higher crude protein, fat, and carbohydrates than Munkoyo. The microbial community composition was also different for the two products, while both are dominated by lactic acid bacteria. Our analyses showed that variation in nutritional composition, defined as how much consumption would contribute to estimated average requirement (EAR), might be explained by variation in microbial community composition. Consumption of Mabisi appeared to contribute more to the estimated average requirement (\% of EAR) and its inclusion in food based recommendations is warranted. We further found evidence that through fermentation of the raw materials (raw milk for Mabisi and cereal for Munkoyo), the levels of B-vitamins can increase. Levels of increase likely depend on the exact composition of the microbial community used for fermentation. Our results show the potential of Mabisi to add value to current diets and suggests that variations in microbial composition between specific product samples can result in variations in nutritional composition.

The in vitro experiment in Chapter $\mathbf{4}$ of this thesis assesses the impact of Mabisi and Munkoyo on the gut microbiota focusing on the potential changes in metabolite profiles of gut microbiota taken from stool samples upon exposure to Mabisi and Munkoyo. The shifts in metabolite profiles were correlated to changes in abundance of a key indicator bacterium (Lactobacillus) for healthy gut microbiota composition. We exposed stool samples to these products and various controls and measured concentrations of short chain fatty acids (SCFAs) and used these as an indicator of beneficial bacteria 
activity and measured shifts in levels of the indicator beneficial bacterium Lactobacillus. Results show that exposure of the gut microbiota from stool to Mabisi and Munkoyo results in higher levels of SCFAs and also higher levels of Lactobacillus. These results support the idea that consumption of fermented foods can result in healthier metabolism of the gut microbiota as measured with SCFA concentrations. These results can inform further more complex in-vitro as well as in vivo studies on the effects of the traditional fermented foods on gut microbiota metabolism and composition.

Chapter 5 describes a survey that was undertaken in Namwala and Mkushi to determine the effect of consuming traditional fermented foods on gut microbiota composition and nutritional status in children 6-24 months old in rural Zambia. Data on food consumption, morbidity and sociodemographic together with stool samples were collected from children aged 6-24 months residing in Namwala and Mkushi in Zambia. The stool samples were analysed for the composition of gut microbiota and for concentrations of short chain fatty acids (SCFAs) as a proxy for microbial metabolism. This data was then linked to data on intake of traditional fermented foods using multivariate analysis of variance. Gut microbiota of children who are fermented beverages consumers was associated with higher relative abundance of Bacteroides and Lactobacillus than the nonconsumers. Higher levels of these bacterial groups have been associated with benefits to the host. There was no difference for the SCFA concentrations between the fermented foods consumers and non-consumers, which may be because other parts of the diets that we did not control for also contribute to SCFA production. The results imply that consumption of the two traditional fermented foods used in this study promotes a healthier gut microbiota composition in the children in Zambia. Our results warrant future more formal tests such as controlled human feeding trials to further validate our findings.

In the framework of the work in this Thesis it is evident the two products are good sources of nutrients and have potential probiotic effects to confer better health and nutritional status to its consumers. The goal of contributing to improvement of people's lives through dietary intake improvements using traditional fermented foods could be achieved as shown in this thesis. The work highlights the need to explore locally available and culturally accepted foods, more so the fermented foods such as Mabisi and Munkoyo in the fight against undernutrition. However further work to generate more evidence for the formalization of our products Mabisi and Munkoyo with key messages on benefits formulated for dissemination to the current and potential consumers. 


\section{Acknowledgements}

It would not have been possible to do the work and eventually write this PhD thesis without the help and support of the kindest people around me. A lot of people have supported me spiritually, emotionally, materially and with the content of this work but it is only possible to give particular attention here to some.

Firstly I wish to say a big thank you to Sijmen, my daily supervisor who mentored me through and through. You were there to give me support and encouragement even when I seemed to have lost direction. Thank you for all the support, the knowledge and believing in me. Special thanks to Bas, my promotor, for also the encouragements and creating time for me in your very busy schedule whenever I need to consult. A big thank you to Elise for the support even when you came in almost midway through my work. You made sure the feedback came my way as quickly as possible and that the nutritional aspect of the work came out as it should. Thank you very much to Inge for pushing the work in the right direction from the initial stage and for the feedback you gave me. To all my promotors, you were so friendly, supportive and offloaded the wisdom and knowledge to me willingly and made sure I felt at home whenever I was doing my work. I would also like to thank my local supervisor, Ray at Tropical Diseases Research Centre (TDRC) for the support and making sure that my work was supported and my travels were facilitated. You made it easier for me to run around collecting data and samples from different areas of Zambia.

I would like to thank my all weather friends, Anneloes, Bernard and Sydney. You guys were great all the time during work and during leisure time. You made the work easier with your encouragements and support. I also wish to thank my students, Marith, Thandie and Guadalupe. You did a great job both going out in the villages of Zambia collecting data and in the laboratory, sometimes spending long hours trying to make things work. I hope the experiences and the knowledge you gained were as helpful to you then and also in your future endeavors as it has been to me. I also sincerely thank Joost for all the help with data manipulations and with the pipeline. You gave me feedback in a timely manner whenever I called upon you.

During my project I worked with other partner institutions. As such, I would like to thank John, Pamela and Vincent from the Department of Food Science and Nutrition at the University of Zambia for their support. Also I thank lan, Sinkala and Banda from the food sciences laboratory, Department of Food Science and Nutrition at the University of Zambia for the help with laboratory work to analyze munkoyo 
and mabisi samples. I thank Phidelis and Jay for the support with experiments and laboratory work at the TDRC. I would not have managed doing the experiments alone especially that it needed me coming at awkward hours to do sampling and freezing the samples. You guys were awesome with the work in the laboratory. I also thank other colleagues at TDRC in the laboratory and in other departments for all the help to facilitate my work.

In Wageningen at the laboratory of genetics, I met and worked with many colleagues but I can only mention a few. A big thank you to Bertha, Corie, Frank and Marijke; you showed me around the laboratories and gave me guidance of how to do certain procedures. I would also like to thank Alex, Jianhua, Claudio, Eric, Sabine, Florin, Suzette, Mariska, Mathijs, Valeria, Ben, Tom, Rene, Krithi, Kim, Margo, Cris, Gabrielle, Jitpanu, Lennart, Ramon, Mark Z, Arjan, Mark A, Raphael, Duur, Jelle, Robert, Fons, Sara, Diego, Bart, Joost and Anna for all the help and the great atmosphere. Special thanks to Wytske for all the help in ensuring that I settled and had all I needed throughout my work. I would like to thank Karin from the Division of Human nutrition and health for the help with nutrition data analysis and advice.

Thank you Bernard and Kim for accepting to stand beside me as my paranymphs. You guys are great and I am indebted to you.

I would like to thank also the Zambian family I found the first time I came to Wageningen. I thank Ashley, Lazarous, Misheck and Allan. You guys received me and showed me around not only Wageningen but other cities in the Netherlands. I also thank the other colleagues I met while in Wageningen, Concilia, Justina, Siatwiinda, Kalima and Marrian. You guys were awesome and made the stay easier.

I would like to thank my spiritual family at Evangel Church of God under Pastor Ndolo for their prayers and encouragement they gave me throughout my work. I also thank my other spiritual family at Amazing Grace Parish (AGP) Wageningen for the prayers and encouragements more so to Pastor Farai and the wife whenever I was in Wageningen.

I most sincerely thank my family. My wife, Suya, you have been by my side with patience and taking care of the children whenever I was away collecting data within Zambia and also in Wageningen. You are a strong woman and I salute you. I also would like to sincerely thank my children, Bwembya, Madaliso and Katongo for the love, support and understanding the nature of my job and also the work for this PhD thesis. May this be an inspiration to you. Thank you also to Mum and Dad for your support and also to all my siblings, relatives and friends. 


\section{List of publications}

Justin Chileshe, Elise F Talsma, Sijmen E Schoustra, Karin J Borgonjen-van den Berg, Ray Handema, Bas J Zwaan and Inge $D$ Brouwer. Potential contribution of cereal and milk based fermented foods to dietary nutrient intake of 1-5 years old children in Central province in Zambia. Submitted

Justin Chileshe, Joost van den Heuvel, Ray Handema, Elise F. Talsma, Bas J Zwaan and Sijmen Schoustra. Nutritional composition and microbial communities of two non-alcoholic traditional fermented beverages from Zambia: A study of Mabisi and Munkoyo. Submitted 


\section{About the Author}

Justin Chileshe was born on 11 August 1971 in Chililabombwe, one of the mining towns of Zambia on the Copperbelt province. He went to Chikola Secondary School in Chingola and completed his secondary education in 1991 and was admitted to the University of Zambia, School of Natural Sciences in 1993 to pursue a Bachelor of Science degree in Chemistry.

He was employed by Gamma Pharmaceuticals limited company from

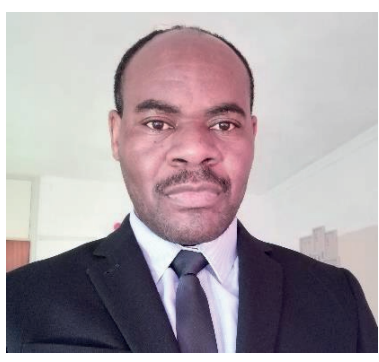
1997 to 2000 as a Quality Control Chemist, working to ensure that quality is maintained for the entire production process of all pharmaceutical products that were being produced. In the year 2000 he moved to the Tropical Diseases Research Centre (TDRC) employed as a Staff Development Fellow. He started learning the art of conducting research while being involved in a number of clinical trials of antimalarial drugs and surveys to determine micronutrient status of populations in Zambia under the leadership of the late Dr. Emmanuel Kafwembe (former TDRC Director).

He was later granted a scholarship to pursue a European Master of Science degree in Food Science, Technology and Nutrition in 2006 at Dublin Institute of Technology in Dublin, the Republic of Ireland. This course was organized to follow different modules also in different countries including Belgium, Portugal and Germany. He completed in 2008 and came back to the TDRC to take up a position as Head of Nutrition and Clinical Chemistry unit to spearhead all research related to nutrition.

In 2013, Justin was introduced to Dr. Sijmen Schoustra by the Deputy Director of TDRC, Dr. Ray Handema, and got interested in research on Zambian traditional non-alcoholic fermented foods. He worked with Dr. Schoustra and Dr. Handema to apply for a grant from Nutricia foundation of the Netherlands, to add the nutritional aspects of the Zambian traditional fermented foods to the WOTRO project that Dr. Schoustra had received. This gave way for Justin in 2015 to pursue the PhD at Wageningen University and Research in the Laboratory of Genetics and collaboration with the Division of Human Nutrition and Health. The work that was done birthed this thesis for Justin. 


\section{Overview of completed training activities}

\section{PE\&RC Training and Education Statement}

With the training and education activities listed below the PhD candidate has complied with the requirements set by the C.T. de Wit Graduate School for Production Ecology and Resource Conservation (PE\&RC) which comprises of a minimum total of 32 ECTS ( $=22$ weeks of activities)

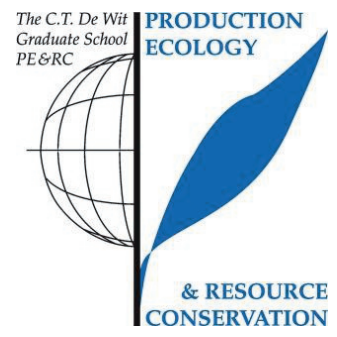

\section{Review of literature (6 ECTS)}

- Potential of non-alcoholic fermented beverages consumed on a regular basis to promote health in Zambia; a study of microbial ecology and nutritional aspects (2015)

\section{Writing of project proposal (4.5 ECTS)}

- Potential of non-alcoholic fermented beverages consumed on a regular basis to promote health in Zambia; a study of microbial ecology and nutritional aspects (2015)

\section{Post-graduate courses (5.1 ECTS)}

- Nutriscience, a multifaceted approach to nutrition research; VLAG (2015)

- Introduction to R for statistical analysis; PE\&RC (2015)

- Exposure assessment in nutrition research; VLAG (2016)

- Epigenesis and epigenetics; VLAG (2017)

\section{Laboratory training and working visits (4.5 ECTS)}

- Working visit for theoretical and laboratory work; University of Zambia (2016)

\section{Deficiency, refresh, brush-up courses (7.5 ECTS)}

- Basic statistics; PE\&RC (2015)

- Genetic analysis, tools and concepts; PE\&RC (2015) 


\section{Competence strengthening / skills courses (0.9 ECTS)}

- Information literacy including EndNote introduction; Wageningen UR Library (2015)

- WGS PhD Workshop carousel; PE\&RC (2015)

PE\&RC Annual meetings, seminars and the PE\&RC weekend (2.4 ECTS)

- PE\&RC First years weekend (2015)

- PE\&RC Day (2015-2017)

- PE\&RC Midterm weekend (2016)

\section{Discussion groups / local seminars / other scientific meetings (4.5 ECTS)}

- Wageningen Evolution and Ecology Seminars (WEES) (2015-2018)

- Microbial population biology series (2017-2018)

International symposia, workshops and conferences (6.6 ECTS)

- WOTRO Dissemination meeting on fermented foods and alleviation of malnutrition and business development; Lusaka, Zambia (2016)

- International symposium on understanding the double burden of malnutrition for effective interventions; Vienna, Austria (2018)

- Evidence for impact symposium: translating research into gains for primary and community health in Zambia; Lusaka, Zambia (2018)

\section{Supervision of MSc students (6 ECTS)}

- Optimizing local diets for 12-60 months old Zambian (Mkushi) children

- Effect of dietary intake and intestinal parasites on the nutritional status of children aged 6-23 months in Namwala and Mkushi districts of Zambia 


\section{Colophon}

The research of this thesis was carried out at the Laboratory of Genetics at Wageningen University, Wageningen, The Netherlands and at the Tropical Diseases Research Centre, Ndola, Zambia as part of the project on Zambian traditional fermented products, which was financially supported by the Nutricia foundation (grant number 2015-51), The Netherlands and Netherlands Organization for Scientific Research Science for Global Development Division (grant number WOTRO 08.250.2013.108).

This project is a collaboration within Wageningen University \& Research with colleagues from the Division of Human Nutrition and Health, as well as with other partners in the Netherlands (CSK Food Enrichment and Yoba-for-Life) and in Zambia (University of Zambia, Tropical Diseases Research Centre and Heifer International).

Cover design by: Proefschriftmaken Thesis layout by: Justin Chileshe

Printed by: Proefschriftmaken 



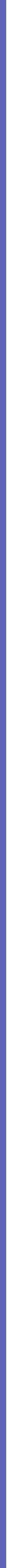

